\title{
Optomagnetismo Associado ao Spin Eletrônico em Semicondutores
}

\author{
Renan Carlos Cordeiro
}

Orientador: Prof.Dr.André Bohomoletz Henriques

Dissertação apresentada ao Instituto de Física da Universidade de São Paulo para a obtenção do título de Mestre em Ciências.

Área de Concentração: Materiais magnéticos e propriedades magnéticas

Banca Examinadora:

Prof. Dr. André Bohomoletz Henriques (IFUSP)

Prof. Dr. Sylvio Roberto Accioly Canuto (IFUSP)

Prof. Dr. Paulo Sérgio Soares Guimarães (UFMG)

São Paulo

2015 
FICHA CATALOGRÁFICA

Preparada pelo Serviço de Biblioteca e Informação do Instituto de Física da Universidade de São Paulo

Cordeiro, Renan Carlos

Optomagnetismo associado ao spin eletrônico em semicondutores. São Paulo, 2015

Dissertação (Mestrado) - Universidade de São Paulo. Instituto de Física. Depto. Física dos Materiais e Mecânica

Orientador: Prof. Dr. André Bohomoletz Henriques

Área de Concentração: Materiais Magnéticos e Propriedades Magnéticas

Unitermos: 1. Optomagnetismo; 2. Ilhas quânticas;

3. Spin eletrônico; 4. Semicondutores; 5. Rotação de Faraday.

USP/IF/SBI-059/2015 
Então a raposa apareceu.

"Bom dia", disse a raposa.

"Bom dia", o Pequeno Príncipe respondeu educadamente.

"Quem é você? Você é tão bonita de se olhar."

"Eu sou uma raposa", disse a raposa.

"Venha brincar comigo", propôs o Pequeno Príncipe. "Eu estou tão triste".

"Eu não posso brincar com você", a raposa disse. "Eu estou cativada".

"O que significa isso - cativar?"

"É uma coisa que as pessoas frequentemente negligenciam", disse a raposa.

"Significa estabelecer laços".

"Sim"disse a raposa. "Para mim você é apenas um menininho e eu

não tenho necessidade de você. E você por sua vez, não tem nenhuma

necessidade de mim. Para você eu não sou nada mais do que uma raposa, mas

se você me cativar então nós precisaremos um do outro".

A raposa olhou fixamente para o Pequeno Príncipe durante muito tempo e disse: "Por favor cativa-me."

"O que eu devo fazer para cativar você??"perguntou o Pequeno Príncipe.

"Você deve ser muito paciente". Disse a raposa. "Primeiro você vai sentar a uma pequena distância de mim e não vai dizer nada. Palavras são as fontes de desentendimento. Mas você se sentará um pouco mais perto de mim todo dia."

Então o Pequeno Príncipe cativou a raposa e depois chegou a hora da partida dele - "Oh!"disse a raposa. "Eu vou chorar".

"A culpa é sua", disse o Pequeno Príncipe,

"mas você mesma quis que eu a cativasse".

"Adeus", disse o Pequeno Príncipe.

"Adeus", disse a raposa.

"E agora eu vou contar a você um segredo: nós só podemos ver perfeitamente com o coração; o que é essencial é invisivel aos olhos. Os homens têm esquecido esta verdade. Mas você não deve esquecê-la.

Você se torna eternamente responsável por aquilo que cativa."

Antoine de Saint-Exupéry, Trecho de "O Pequeno Príncipe" 



\section{Agradecimentos}

Ao Prof. Dr. André Bohomoletz Henriques, orientador, pela orientação durante o mestrado e pelo meu desenvolvimento profissional/pessoal;

Aos meus pais, Elisabete Aparecida Barbedo Cordeiro e Luiz Carlos Cordeiro, pelo apoio, carinho, dedicação, incentivo e ensinamentos que formaram o que sou hoje. Meus pilares de vida;

A toda minha família, que sempre se demonstrou disposta a me incentivar em minhas decisões;

Ao Instituto de Física da USP, pelo fornecimento da infraestrutura necessária à realização do mestrado;

Ao CNPq (Conselho Nacional de Desenvolvimento Científico e Tecnológico) pelo apoio concedido durante o período de realização deste trabalho;

Aos funcionários do Departamento de Física dos Materiais e Mecânica, pelos serviços e atendimentos prestados;

Aos meus amigos do Instituto de Física, Eduardo Sell Gonçalves, Gabriel Marinello, Francielle A. C. Sezotzki, Natacha Enoki, Rafael Martinez de Araujo, Tibério Ferrari, Flávio C. de Moraes, entre muitos outros, por me incentivarem na constituição desse trabalho;

Às minhas amigas desde tempo de colégio, Carolina Yosino, Laís Emy Ishibashi, Patrícia Schlithler e Beatriz Beres Meira, pela incrível companhia fornecida;

À Alisson Luís Soares Teixeira, pelos incríveis momentos proporcionados e por sua presença única e motivadora nos momentos mais marcantes deste último ano;

À Lucas Barboza Martins, Mario Henrique Germoliato, Berg Pessoa, Yuri Belmock, Luís Matinha, Matheus Vidal, Matheus Rosa, Lucas Maia, Gil Cardoso, Henrique Furquim, Alex Barreto, entre muitos outros, por mostrarem o grau de importância da amizade e a forma como a mesma supera obstáculos;

E a todos aqueles que colaboraram e contribuíram de alguma forma, seja pessoal ou profissional, para a concretização desse trabalho; 


\section{RESUMO}

O spin de um elétron confinado em uma ilha quântica (do inglês, quantum dot ou QD) oferece a oportunidade de armazenamento e manipulação de coerência de fase em escalas de tempo muito mais longas do que aquelas encontradas em dispositivos convencionais. A natureza zero-dimensional dessas estruturas pode ser explorada em dispositivos optoeletrônicos baseados na manipulação de spin pela luz, tais como QD lasers, emissores de fóton-único e transistores de elétron-único.

Desta maneira, o entendimento da física por trás do controle do magnetismo pela luz torna-se essencial no avanço do campo de manipulação de spin e no desenvolvimento de aparelhos tecnológicos. Em particular, o enfoque dessa tese, se refere à geração induzida de magnetização em um conjunto de ilhas quânticas, mediante a iluminação por um pulso de luz circularmente polarizado ressonante com a energia de transição dos QD's. Neste trabalho em questão, dois modelos quânticos para a magnetização induzida pela luz são apresentados. Para ambos os modelos, a fase de precessão da magnetização em função do campo magnético apresentou excelente concordância com os dados experimentais referentes a um conjunto de ilhas quânticas carregadas de (In, Ga)As. Demonstramos ainda, que a precessão do buraco participante do tríon desempenha um papel fundamental na determinação da amplitude e fase da precessão da magnetização. Ressaltamos também a aplicabilidade do modelo na descrição de ilhas carregadas positivamente. E por fim, sugerimos que a teoria desenvolvida pode ser utilizada como técnica de medição do tempo de vida ressonante do tríon em função da energia de emissão do QD. 


\section{ABSTRACT}

The spin of an electron confined in a quantum dot (QD) offers the opportunity to store and manipulate phase coherence over much longer time scales than it is typically possible in charge based devices. The zero-dimensional nature of these nanostructures can be exploited in optoeletronic devices, such as quantum dot laser, single-photon emitters, single-electron transistor and spin-manipulation.

Thus, understanding the physics behind light control of magnetism is essential to advance this field and device applications based on it. In particular, magnetization generation can be induced in an ensemble of quantum dots, each charged with a single electron, when illuminated with a short circularly polarized light pulse resonant with the fundamental gap of the QDs.

In this work, two quantum-mechanical models for the light-induced magnetization are presented. For both models, the phase of magnetization precession as a function of the strength of the magnetic field in a Voigt geometry is in excellent agreement with experimental data measured on (In, Ga)As singly charged quantum dot ensemble. It is demonstrated that the precession of the hole in the trion plays a vital role because it determines the amplitude and phase of the magnetization precession. The model could also be easily extended to describe positively charged quantum dots. We also suggest that our theory, can be used as technique to measure the resonante trion lifetime as a function of QD emission energy. 


\section{Índice}

Índice $\quad$ iv

1 Introdução 1

2 Fundamentos Teóricos 4

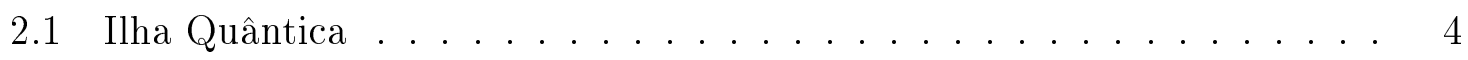

2.2 Níveis de Energia e Regras de Seleção Óptica . . . . . . . . . . . . . 5

2.3 Hamiltoniana de um Elétron em Campo Magnético . . . . . . . . 8

2.4 Interação Hiperfina . . . . . . . . . . . . . . . . . . . . . 9

2.4.1 O Campo Magnético Dipolar . . . . . . . . . . . . . 9

2.4.2 A Interação Quântica Básica . . . . . . . . . . . . . . 11

2.4 .3 A Interação de Contato de Fermi . . . . . . . . . . . . . 13

2.4.4 A Interação Hiperfina de Contato de Fermi em Ilhas Quânticas . 18

2.4.5 Defasagem do Spin Eletrônico devido às Flutuações do Campo Nuclear ........................... 20

2.4.6 Defasagem de Spin Eletrônico na Flutuação Congelada do Campo

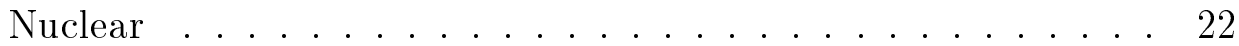

2.5 Rotação de Faraday Resolvida no Tempo . . . . . . . . . . . . . . . . 24

3 Material e Arranjo Experimental $\quad 27$

3.1 Arranjo Experimental . . . . . . . . . . . . . . 27 
3.1.1 Montagem para Fotoluminescência . . . . . . . . . . . 28

3.1.2 Montagem para Rotação de Faraday . . . . . . . . . . . . . . 29

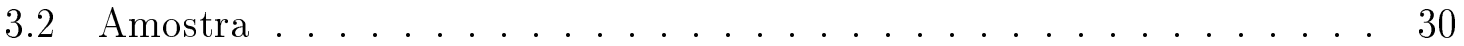

4 Resultados e Análises 32

4.1 Orientação Óptica de Spin em Semicondutores . . . . . . . . . . . . . 32

4.2 Descrição Qualitativa para Geração de Coerência de Spin . . . . . . . . 32

4.3 Modelo Vigente na Descrição de Coerência de Spin para Transições

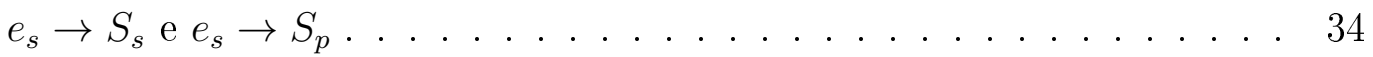

4.4 Efeito de Precessão do Buraco na Polarização do Spin Eletrônico . . . 42

4.5 Modelo do Buraco Estático . . . . . . . . . . . . . . . . . 48

4.6 Resultados Experimentais e Comparação com Modelos Teóricos . . . 51

4.7 Interpretação Física . . . . . . . . . . . . . . . . . . 53

5 Conclusões $\quad 56$

$\begin{array}{lll}6 & \text { Perspectivas Futuras } & 58\end{array}$

A Hamiltoniana Dipolar $\quad 59$

B Identidade Algébrica $\quad 62$

C Sinal TRFR de um Conjunto de Ilhas Quânticas 63

D Artigo Publicado e Apresentações em Conferências $\quad 67$

$\begin{array}{ll}\text { Referências Bibliográficas } & 76\end{array}$ 


\section{Capítulo 1}

\section{Introdução}

A física nos últimos 25 anos vem sofrendo profundas transformações, decorrentes do desenvolvimento de novas técnicas de manipulação da matéria em níveis molecular e atômico. Até o início da década de 1970, os modelos microscópicos da matéria só podiam ser testados em amostras volumétricas. Dentro deste contexto, se desenvolveram as teorias sobre o magnetismo, a supercondutividade, etc[1].

No entanto, a partir de meados dos anos 1970 tornou-se possível a fabricação de estruturas "artificiais"nas quais átomos e moléculas fossem manuseados individualmente, proporcionando, portanto, o surgimento de novos materiais, a exemplo de estruturas cristalinas de baixa dimensionalidade, poços quânticos, ilhas quânticas e filmes-finos. As consequências tecnológicas desses estudos foram igualmente novas, como: obtenção de propriedades metálicas, semicondutoras ou isolantes através de um único nanotubo de carbono; advento de dispositivos eletrônicos, como diodos e transistores, em dimensões moleculares; o desenvolvimento do nanomagnetismo - principal promessa de inovação tecnológica nas próximas décadas - entre muitos outros [1, 2].

Dentro desse contexto, destacam-se os estudos na área de Ilhas quânticas semicondutoras (do inglês, semiconductor quantum dots ou simplesmente QD), cujas pesquisas vem sendo estimuladas e intensificadas por diversos elementos, englobando desde fabricação de QD lasers[3, 4], emissores de fóton-único[5] e transistores de elétron-único[6], 
até o uso de spins isolados para computação quântica[7, 8, 9].

O termo "ilha quântica", amplamente empregado a partir do final dos anos 80, refere-se especificamente a estruturas semicondutoras de escala nanométrica. Os tamanhos típicos de uma ilha quântica compreendem uma região que varre desde poucos nanômetros, em ilhas coloidais (também referidas como nano-cristais), até a algumas centenas deles, para o caso de estruturas eletrostáticas fabricadas litograficamente. O tamanho físico reduzido dessas componentes é a principal característica em comum das ilhas quânticas formadas por diferentes materiais e fabricadas por diferentes técnicas experimentais. Tal aspecto singular dá origem a propriedade básica de maior relevância das ilhas quânticas: a supressão do movimento dos elétrons (e/ou buracos) presentes nessas estruturas. Isto é, o tamanho reduzido dos QD's acarreta no confinamento e localização espacial (em todas as três direções) das partículas residentes em seu interior ${ }^{1}$. Como resultado do confinamento espacial segue a completa quantização (ou discretização) do espectro de energia dos portadores de carga confinados às ilhas quânticas.

O espectro de energia quantizado e a localização espacial se assemelham ao comportamento dos elétrons em um átomo, o que dá origem a designação das ilhas quânticas como "Átomos Artificiais". Em particular, essa última descrição enfatiza e configura o aumento do isolamento dos elétrons (e/ou buracos) confinados às ilhas em relação ao ambiente externo que os cerca. Tal disposição é uma das mais atraentes propriedades exploradas para aplicações[10], que varrem desde QD lasers destinados à área de telecomunicações, até o desenvolvimento de spin-qubit (unidades de informação com qualidade quântica) voltados à tecnologia computacional ${ }^{2}$.

\footnotetext{
${ }^{1}$ Esta propriedade é o que dá às ilhas quânticas o subtítulo de estruturas zero-dimensionais ("0D")

${ }^{2}$ Este é o ponto que mais desperta o fascínio da ciência, um computador quântico não possui qualquer limite teórico para sua capacidade, um único átomo pode abrigar informações infinitamente mais complexas em seu nível quântico do que um bit convencional.
} 
Nesse contexto, uma boa compreensão e estudo quanto aos mecanismos de geração e manipulação ultrarrápida da ordem magnética em semicondutores através de estímulos luminosos (dito optomagnetismo) merece papel de destaque, uma vez que concilia o processamento de informação à altas velocidades para com as capacidades ampliadas de transmissão e armazenamento de dados.

Tendo isso em vista, nos capítulos seguintes são desenvolvidos os modelos do processo de magnetização pela luz em ilhas quânticas de GaAs carregadas com elétrons residentes. Tais modelagens são validadas mediante o confrontamento para com medidas experimentais de Rotação de Faraday Resolvida no Tempo. 


\section{Capítulo 2}

\section{Fundamentos Teóricos}

\subsection{Ilha Quântica}

Uma Ilha Quântica (em inglês, Quantum Dot) é uma nanoestrutura semicondutora que limita o movimento dos elétrons da banda de condução, buracos da banda de valência ou excitons (pares ligados de elétrons da banda de condução e buracos de valência) em todas as três dimensões espaciais. Devido ao processo de confinamento, tais estruturas são também chamadas de átomos artificiais, e são consideradas promissoras candidatas no campo da spintrônica e no desenvolvimento de dispositivos de processamento de informações quânticas.

No contexto da Mecânica Quântica, um elétron é descrito por uma função de onda $\Psi$ a qual obedece a denominada Equação de Schrödinger [11] dada por :

$$
i \hbar \frac{\partial}{\partial t} \Psi=\mathcal{H} \Psi
$$

onde $\hbar$ é a constante de Planck dividida por $2 \pi, t$ o tempo e $\mathcal{H}$ a hamiltoniana do sistema.

Em particular a função de onda pode ser separada em duas composições: a primeira dependente do tempo, $T(t)$, e a segunda dependente das coordenadas espaciais, $\Psi(\boldsymbol{r})$. Assim, segue que a solução para a parte temporal é determinada pela energia $E$ do 
sistema e dada por:

$$
T(t)=\exp \left(-i \frac{E}{\hbar} t\right)
$$

Enquanto que a parte espacial corresponde a uma equação diferencial - denominada Equação de Schrödinger independente do tempo - formulada como:

$$
\left\{-\frac{\hbar^{2}}{2 m_{e}} \nabla^{2}+V(\boldsymbol{r})\right\} \Psi(\boldsymbol{r})=E \Psi(\boldsymbol{r})
$$

onde $m_{e}$ é a massa do elétron, $\boldsymbol{\nabla}$ o operador laplaciano e $V(\boldsymbol{r})$ a energia potencial do sistema.

Particularmente, devido à propriedade de confinamento eletrônico, podemos descrever uma ilha quântica como uma "caixa" cúbica capaz de confinar um elétron em seu interior em todas as dimensões espaciais, numa espécie de estrutura zero dimensional. A solução para a energia do sistema é, portanto, equivalente ao problema de Mecânica Quântica básica e dada por :

$$
E=\frac{\hbar^{2} \pi^{2}}{2 m_{e} a^{2}}\left(n_{x}^{2}+n_{y}^{2}+n_{z}^{2}\right)
$$

onde $a$ é a dimensão da caixa e $n_{x}, n_{y}, n_{z}$ são números inteiros.

Na verdade, os níveis de energia de uma ilha quântica não são tão simples como na equação (2.4), porém as energias calculadas por essa via nos dão uma boa aproximação para o entendimento do confinamento dos elétrons residentes no interior de uma ilha quântica.

\subsection{Níveis de Energia e Regras de Seleção Óptica}

Em particular, os estados de energia de uma ilha quântica são completamente quantizados, de forma tal que excitações e emissões de fótons ocorram somente para determinados valores de energia. A dimensão das ilhas quânticas - da ordem de alguns nanometros - é bastante pequena quando comparada com os padrões macroscópicos, 
no entanto, é grande quando confrontada com a distância interatômica típica presente em estruturas sólidas - da ordem de alguns ângstrons.

Portanto, o estado eletrônico nas ilhas quânticas pode ser descrito como o produto de uma função de Bloch, cuja extensão encontra-se na escala atômica, por uma função do tipo envelope, cuja ordem de grandeza é do tamanho de uma ilha quântica.

As ilhas que fazem parte de nosso interesse apresentam um gap direto, no qual os estados próximos ao mínimo da banda de condução apresentam propriedades equivalentes a um orbital atômico tipo-s, enquanto que os estados próximos ao máximo da banda de valência exibem simetria de orbitais tipo- $p$. A figura 2.1, a seguir, exibe a micrografia obtida por Microscopia Eletrônica de Transmissão (TEM) de uma ilha quântica de InAs/GaAs crescida via Epitaxia por Feixe Molecular (MBE), bem como um esquema simplificado dos níveis de energia presentes em um típico quantum dot.

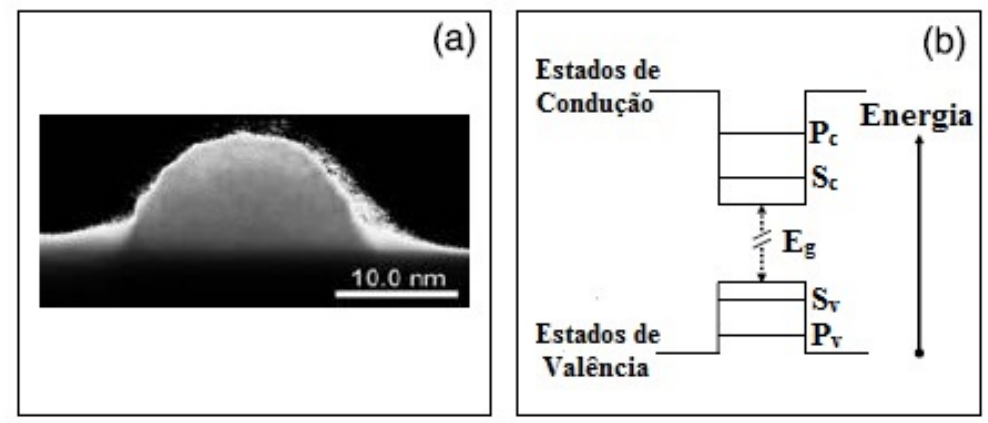

Figura 2.1: (a) Micrografia obtida por microscopia eletrônica de transmissão de uma ilha quântica de InAs/GaAs crescida via epitaxia por feixe molecular[12]; (b) Esquema simplificado dos estados de energia discretos em um quantum dot, onde a separação de energia $E_{g}$ corresponde ao gap energético entre as bandas de condução e valência. As nomenclaturas $S_{c}$ e $P_{c}$ referem-se aos estados da banda de condução que apresentam, respectivamente, simetria tipo-s e tipo-p. O mesmo vale para os estados $S_{v}$ e $P_{v}$ da banda de valência[12].

A interação de uma onda eletromagnética com portadores de carga num semicondutor é governada por rigorosas regras de seleção[13]. Para típicas estruturas cúbicas, tal como a apresentada em GaAs, tanto energia quanto momento angular total são conservados em transições eletrônicas envolvendo as bandas de valência e condução. Como 
mencionado anteriormente, os estados na banda de condução apresentam propriedades semelhantes a orbitais tipo-s (número quântico orbital $l=0$ ), e por consequência, o momento angular total dos elétrons presentes nessa faixa é simplesmente $m_{s}= \pm \frac{1}{2}$ $(\uparrow$ ou $\downarrow)$. Por outro lado, os estados da banda de valência apresentam simetria tipo- $p$ (número quântico orbital $l=1$ ), e portanto, são determinados através do acoplamento spin-órbita. Nesse caso específico, segue que o momento angular total $\boldsymbol{J}=\boldsymbol{L}+\boldsymbol{S}$ exibe as seguintes projeções vetoriais ${ }^{1}: J_{z}^{h}= \pm \frac{3}{2}$ ( $\Uparrow$ ou $\Downarrow$, denominados "buracos pesados") e $J_{z}^{h}= \pm \frac{1}{2}$ (denominados "buracos leves").

Dessa maneira, segue que a absorção de um fóton pode aumentar o momento angular total de um elétron na banda de valência para a banda de condução em $J_{z}^{e}=-J_{z}^{h}+1$, se o fóton for circularmente polarizado para direita, ou reduzir em $J_{z}^{e}=-J_{z}^{h}-1$, se o fóton for circularmente polarizado para esquerda. Tais regras de seleção óptica² encontram-se esquematizadas na figura 2.2 abaixo.

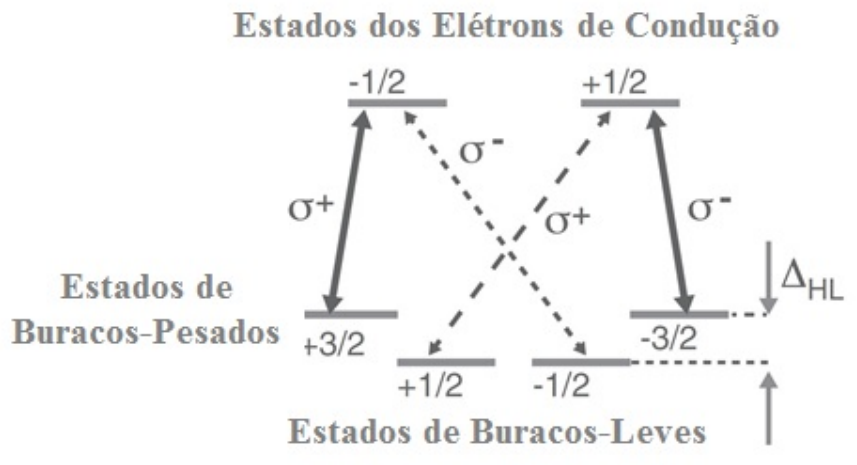

Figura 2.2: Regras de seleção óptica aplicadas às transições do estado fundamental, incluindo a degenerescência da banda de valência. Parte superior corresponde às projeções do momento angular total $J$ dos elétrons fotoexcitados na banda de condução, enquanto a parte inferior representa as projeções $J_{z}$ dos buracos na banda de valência.[10]

No caso em particular, os estados de valência de buracos-pesados e buracos-leves são separados por uma energia $\Delta_{H L}$ devido aos efeitos de tensão (em inglês, strain)

\footnotetext{
${ }^{1} \mathrm{O}$ eixo $z$ de quantização é escolhido perpendicular ao plano da ilha quântica e na maioria dos experimentos também é paralelo à direção de propagação do feixe de excitação.

${ }^{2}$ As regras de seleção para absorção e emissão de fótons são idênticas.
} 
e confinamento quântico. Na prática, os efeito de tensão, anisotropia de forma, entre outros, podem introduzir o acoplamento dos buracos-leves aos pesados, de forma tal que todas as transições indicadas na figura 2.2 sejam possíveis entre os estados mistos, com diferentes probabilidades de ocorrência.

\subsection{Hamiltoniana de um Elétron em Campo Magné- tico}

Uma partícula carregada girando em uma órbita circular constitui um dipolo magnético. Seu momento de dipolo magnético, $\boldsymbol{\mu}$, é proporcional ao seu momento angular intrínseco de spin, $\boldsymbol{S}$ por uma constante multiplicativa $\gamma$ denominada razão giromagnética [11], ou seja:

$$
\boldsymbol{\mu}=\gamma \boldsymbol{S}
$$

Quando um dipolo magnético $\boldsymbol{\mu}$ é posicionado em um campo magnético externo $\boldsymbol{B}$, o mesmo experimenta um torque da forma $\boldsymbol{\mu} \times \boldsymbol{B}$, o qual tende a alinhá-lo paralelo ao campo.

Para calcularmos a energia potencial associada a esse sistema precisamos primeiro fazer algumas considerações. Inicialmente consideremos um campo $\boldsymbol{B}$ uniforme e apontando ao longo da direção $y$, conforme apresentado na figura 2.3. Sem perda de generalidade, podemos considerar que o momento magnético vindo do infinito encontra-se paralelo ao eixo $x$ e que ao ser levado até a origem do sistema de coordenadas sofre uma rotação até chegar numa posição de ângulo $\theta$ para com o eixo vertical.

Nessa situação o torque exercido por $\boldsymbol{B}$ é $\boldsymbol{\tau}=\boldsymbol{\mu} \times \boldsymbol{B}=\mu B \operatorname{sen} \theta \hat{z}$ e consequentemente o trabalho realizado até a chegada nessa configuração pode ser descrito por:

$$
\begin{gathered}
U=\int_{\pi / 2}^{\theta} \mu B \operatorname{sen} \theta^{\prime} d \theta^{\prime}=\mu B\left(-\cos \theta^{\prime}\right)_{\pi / 2}^{\theta}=-\mu B(\cos \theta-\cos (\pi / 2)) \\
U=-\mu B \cos \theta \Rightarrow U=-\boldsymbol{\mu} \cdot \boldsymbol{B}
\end{gathered}
$$




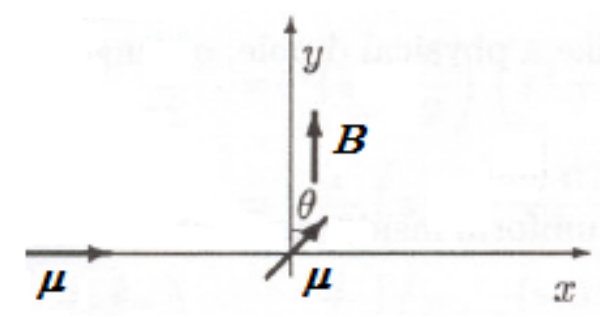

Figura 2.3: Figura esquemática para o cálculo da energia potencial de um dipolo magnético em um campo uniforme $\boldsymbol{B}$. O momento magnético $\boldsymbol{\mu}$ parte do infinito apontando paralelamente ao eixo $x$ e sofre uma rotação mediante o torque exercido por $\boldsymbol{B}$ até chegar numa configuração de ângulo $\theta$ para com o eixo vertical [14].

Finalmente, concluímos que a hamiltoniana capaz de descrever a interação de um dipolo magnético em um campo externo $\boldsymbol{B}$ é dada por:

$$
\mathcal{H}=-\boldsymbol{\mu} \cdot \boldsymbol{B}
$$

\subsection{Interação Hiperfina}

A Interação Hiperfina é proveniente do acoplamento do momento magnético eletrônico para com o momento magnético nuclear, e pode ser visualizada como o movimento de um elétron na presença do campo magnético do núcleo. Nessas condições, é razoável supor que a interação ocorre de forma análoga a um par de dipolos magnéticos, cuja abordagem será melhor desenvolvida nas passagens seguintes.

\subsubsection{O Campo Magnético Dipolar}

De acordo com o eletromagnetismo clássico, o termo dominante na expansão em multipolos magnéticos do potencial vetor $\boldsymbol{A}$ pode ser expresso ${ }^{3}$ em termos do momento magnético $\boldsymbol{\mu}$ como:

$$
\boldsymbol{A}=\frac{\mu_{0}}{4 \pi} \frac{\boldsymbol{\mu} \times \boldsymbol{r}}{r^{3}}
$$

\footnotetext{
${ }^{3}$ em unidades do Sistema Internacional (S.I.)
} 
onde $\mu_{0}$ consiste da permeabilidade magnética no vácuo, e $\boldsymbol{r}$ corresponde ao vetor posição do ponto de observação [15].

Consequentemente, o campo magnético devido ao dipolo $\boldsymbol{\mu}$ pode ser calculado considerando-se o rotacional da equação (2.8). Para tanto, usando-se da seguinte identidade vetorial $\boldsymbol{\nabla} \times(\boldsymbol{A} \times \boldsymbol{B})=\boldsymbol{A}(\boldsymbol{\nabla} \cdot \boldsymbol{B})-\boldsymbol{B}(\boldsymbol{\nabla} \cdot \boldsymbol{A})+(\boldsymbol{B} \cdot \boldsymbol{\nabla}) \boldsymbol{A}-(\boldsymbol{A} \cdot \boldsymbol{\nabla}) \boldsymbol{B}$, temos que

$$
\begin{aligned}
\boldsymbol{\nabla} \times(\boldsymbol{\mu} \times \boldsymbol{r}) & =(\boldsymbol{\nabla} \cdot \boldsymbol{r}) \boldsymbol{\mu}+(\boldsymbol{r} \cdot \boldsymbol{\nabla}) \boldsymbol{\mu}-(\boldsymbol{\nabla} \cdot \boldsymbol{\mu}) \boldsymbol{r}-(\boldsymbol{\mu} \cdot \boldsymbol{\nabla}) \boldsymbol{r} \\
& =3 \boldsymbol{\mu}-(\boldsymbol{\mu} \cdot \nabla) \boldsymbol{r}
\end{aligned}
$$

uma vez que $\boldsymbol{\nabla} \cdot \boldsymbol{r}=3$, e que qualquer derivada espacial de $\mu$ é igual a zero.

Além disso, como

$$
\begin{aligned}
(\boldsymbol{\mu} \cdot \nabla) \boldsymbol{r} & =\left\{\mu_{x} \frac{\partial}{\partial x}+\mu_{y} \frac{\partial}{\partial y}+\mu_{z} \frac{\partial}{\partial z}\right\}(x \hat{\boldsymbol{x}}+y \hat{\boldsymbol{y}}+z \hat{\boldsymbol{z}}) \\
& =\mu_{x} \hat{\boldsymbol{x}}+\mu_{y} \hat{\boldsymbol{y}}+\mu_{z} \hat{\boldsymbol{z}}=\boldsymbol{\mu}
\end{aligned}
$$

Segue que $\boldsymbol{\nabla} \times(\boldsymbol{\mu} \times \mathbf{r})=3 \boldsymbol{\mu}-\boldsymbol{\mu}=2 \boldsymbol{\mu}$, do qual obtém-se que ${ }^{4}$

$$
\begin{aligned}
\boldsymbol{B} & =\boldsymbol{\nabla} \times \boldsymbol{A}=\frac{\mu_{0}}{4 \pi}\left[-\frac{3}{r^{5}} \boldsymbol{r} \times(\boldsymbol{\mu} \times \boldsymbol{r})+\frac{2 \boldsymbol{\mu}}{r^{3}}\right] \\
& =\frac{\mu_{0}}{4 \pi} \frac{1}{r^{5}}\left[-3 \boldsymbol{r} \times(\boldsymbol{\mu} \times \boldsymbol{r})+2 r^{2} \boldsymbol{\mu}\right]
\end{aligned}
$$

Finalmente, usando-se de que $\boldsymbol{r} \times(\boldsymbol{\mu} \times \boldsymbol{r})=(\boldsymbol{r} \cdot \boldsymbol{r}) \boldsymbol{\mu}-(\boldsymbol{r} \cdot \boldsymbol{\mu}) \boldsymbol{r}=r^{2} \boldsymbol{\mu}-(\boldsymbol{r} \cdot \boldsymbol{\mu}) \boldsymbol{r}$ concluímos que o campo devido a um dipolo magnético é descrito por:

$$
\boldsymbol{B}=\frac{\mu_{0}}{4 \pi} \frac{1}{r^{5}}\left[3(\boldsymbol{r} \cdot \boldsymbol{\mu}) \boldsymbol{r}-r^{2} \boldsymbol{\mu}\right]
$$

Em posse desse resultado (2.12), segue que a energia potencial de interação $\mathcal{E}$ entre dois momentos magnéticos $\boldsymbol{\mu}_{1}$ e $\boldsymbol{\mu}_{2}$ é dada por meio do produto escalar do campo

\footnotetext{
${ }^{4}$ Aqui empregou-se a seguinte identidade vetorial:
}

$$
\boldsymbol{\nabla} \times(\psi \boldsymbol{A})=\boldsymbol{\nabla} \psi \times \boldsymbol{A}+\psi \boldsymbol{\nabla} \times \boldsymbol{A}
$$


magnético gerado pelo primeiro $\left(\boldsymbol{B}_{\mathbf{1}}\right)$ para com o momento de dipolo do segundo $\left(\boldsymbol{\mu}_{2}\right)$, ou seja, $\mathcal{E}=-\boldsymbol{\mu}_{\mathbf{2}} \cdot \boldsymbol{B}_{1}$ - vide equação (2.7). Por consequência, concluímos que

$$
\mathcal{E}=\frac{\mu_{0}}{4 \pi}\left\{\frac{\boldsymbol{\mu}_{\mathbf{1}} \cdot \boldsymbol{\mu}_{\mathbf{2}}}{r^{3}}-\frac{3\left(\boldsymbol{\mu}_{\mathbf{1}} \cdot \boldsymbol{r}\right)\left(\boldsymbol{\mu}_{\mathbf{2}} \cdot \boldsymbol{r}\right)}{r^{5}}\right\}
$$

onde $\boldsymbol{r}$ corresponde ao vetor que conecta ambos os momentos de dipolo mencionados.

\subsubsection{A Interação Quântica Básica}

A equação (2.12) expressa o campo magnético gerado por uma partícula que possui momento magnético dipolar puro. Tal relação tem seu regime de validade restrito a regiões suficientemente distantes, nas quais os momentos magnéticos de ordem superior ao dipolar apresentam contribuições desprezíveis em relação a esse último. Sendo assim, segundo (2.13), a hamiltoniana que descreve a interação entre um núcleo atômico e um elétron, suficientemente afastados um do outro, é dada por:

$$
\mathcal{H}=\frac{\mu_{0}}{4 \pi}\left\{\frac{\boldsymbol{\mu}_{\boldsymbol{e}} \cdot \boldsymbol{\mu}_{\boldsymbol{n}}}{r^{3}}-\frac{3\left(\boldsymbol{\mu}_{\boldsymbol{e}} \cdot \boldsymbol{r}\right)\left(\boldsymbol{\mu}_{\boldsymbol{n}} \cdot \boldsymbol{r}\right)}{r^{5}}\right\}
$$

Onde $\boldsymbol{\mu}_{\boldsymbol{e}}=-g_{e} \mu_{B} \boldsymbol{I}_{\boldsymbol{e}} / \hbar$ é o momento magnético do elétron; $\boldsymbol{\mu}_{\boldsymbol{n}}=g_{n} \mu_{n} \boldsymbol{I}_{\boldsymbol{n}} / \hbar$ é o momento magnético do núcleo; $\mu_{B}$ é o magneton de Bohr; $\mu_{n}$ é o magneton nuclear; $\boldsymbol{r}$ corresponde ao vetor que conecta ambos os momentos de dipolo mencionados; $\boldsymbol{I}_{\boldsymbol{e}}$ representa o operador de spin do elétron; $\boldsymbol{I}_{\boldsymbol{n}}$ corresponde ao operador de spin nuclear; $g_{e}$ e $g_{n}$ são, nessa ordem, os fatores giromagnéticos eletrônico e nuclear [16].

Escrevendo-se $\boldsymbol{\mu}_{\boldsymbol{e}}$ e $\boldsymbol{\mu}_{\boldsymbol{n}}$ em componentes cartesianas, segue que a hamiltoniana (2.14) apresenta termos da forma:

$$
-g_{e} g_{n} \frac{\mu_{B} \mu_{n}}{\hbar^{2}} I_{e x} I_{n x} \frac{1}{r^{3}} \quad, \quad-g_{e} g_{n} \frac{\mu_{B} \mu_{n}}{\hbar^{2}} I_{e x} I_{n y} \frac{x y}{r^{3}}
$$

Se expressarmos $I_{e x}$ e $I_{e y}$ em termos dos operadores de abaixamento e levantamento, respectivamente $I_{e}^{-}$e $I_{e}^{+}$, descrevermos os operadores $I_{n x}$ e $I_{n y}$ em função de $I_{n}^{+} \mathrm{e}$ 
$I_{n}^{-}$, e escrevermos as coordenadas cartesianas $x, y$ e $z$ em termos das coordenadas esféricas, podemos desenvolver a hamiltoniana (2.14) em uma forma particularmente conveniente $^{5}$

$$
\mathcal{H}=-\frac{\mu_{0}}{4 \pi} \frac{g_{e} g_{n} \mu_{B} \mu_{n}}{\hbar^{2} r^{3}}(A+B+C+D+E+F)
$$

na qual

$$
\left\{\begin{array} { l } 
{ A = I _ { e z } I _ { n z } ( 1 - 3 \operatorname { c o s } ^ { 2 } \theta ) } \\
{ B = - \frac { 1 } { 4 } ( I _ { e } ^ { + } I _ { n } ^ { - } + I _ { e } ^ { - } I _ { n } ^ { + } ) ( 1 - 3 \operatorname { c o s } ^ { 2 } \theta ) } \\
{ C = - \frac { 3 } { 2 } ( I _ { e } ^ { + } I _ { n z } + I _ { e z } I _ { n } ^ { + } ) \operatorname { s e n } \theta \operatorname { c o s } \theta e ^ { - i \phi } }
\end{array} \left\{\begin{array}{l}
D=-\frac{3}{2}\left(I_{e}^{-} I_{n z}+I_{e z} I_{n}^{-}\right) \operatorname{sen} \theta \cos \theta e^{i \phi} \\
E=-\frac{3}{4} I_{e}^{+} I_{n}^{+} \operatorname{sen}^{2} \theta e^{-2 i \phi} \\
F=-\frac{3}{4} I_{e}^{-} I_{n}^{-} \operatorname{sen}^{2} \theta e^{2 i \phi}
\end{array}\right.\right.
$$

Desta maneira, calculemos a energia potencial de interação magnética considerandose um sistema elétron-núcleo, no qual (2.14) atua como uma perturbação sobre os orbitais atômicos. Nessa situação, enquanto a função de onda eletrônica for um estado tipo- $p$, tipo- $d$, ou qualquer outro estado de momento angular não nulo, esperamos que (2.14) seja uma boa aproximação no tratamento da interação, uma vez que nesses casos, as funções eletrônicas se anulam em $r=0$, e portanto, a probabilidade de o elétron ser encontrado na região próxima ao núcleo é bastante diminuta. Para estados tipo-s, entretanto, a função de onda eletrônica é não-nula na origem e nessa situação de curtas distâncias a aproximação dipolar torna-se suspeita.

Em particular, quando a distância entre as partículas for pequena a ponto de os momentos magnéticos de ordem superior tornarem-se relevantes, a interação dada por (2.14) torna-se inexata na descrição da interação magnética entre o núcleo atômico e o elétron. Uma observação mais cuidadosa desse fato evidencia a imprecisão de (2.14). Para tanto, suponhamos um valor médio de $\mathcal{H}$ sobre uma função eletrônica tipo- $s, u(r)$, como é usual no cálculo de teoria de perturbação em primeira-ordem. Processando-se

\footnotetext{
${ }^{5}$ Para maiores detalhes vide Apêndice A.
} 
desta maneira, há um número de termos de (2.14) similares aos termos $A, B, C, D$, $E$ e $F$ como apresentados na equação (2.16). Sendo assim, escolhamos o termo $A$, por exemplo, o qual depende dos parâmetros de ângulo e distância. Logo, a menos de constantes multiplicativas, o valor médio de tal termo é dado por:

$$
\int \frac{u^{2}(r)}{r^{3}}\left(1-3 \cos ^{2} \theta\right) r^{2} d r d \Omega
$$

onde $d \Omega$ é o elemento infinitesimal de ângulo sólido.

Nesse momento nos deparamos com uma situação singular. Se calcularmos a integração angular primeiro, o resultado final para (2.18) é zero, tendo em vista que

$$
\iint\left(1-3 \cos ^{2} \theta\right) \operatorname{sen} \theta d \theta d \phi=(2 \pi) \int_{0}^{\pi}\left(1-3 \cos ^{2} \theta\right) d \theta=(2 \pi) \int_{-1}^{1}\left(1-3 x^{2}\right) d x=0
$$

Por outro lado, se integrarmos primeiro sobre $r$, encontramos um problema na proximidade de $r \simeq 0$, onde $u^{2}(0) \neq 0$, tendo em vista que, nessa região, a integração resulta em função logarítmica e ocasiona em um resultado infinito para (2.18).

Desta maneira, como podemos obter zero ou infinito dependendo da ordem de integração empregada, torna-se claro que não podemos simplesmente ignorar as contribuições de quando $r$ é pequeno.

Portanto, torna-se evidente que a aproximação dipolar é inadequada para a descrição da interação magnética elétron-núcleo na situação em que a função eletrônica não se anula na posição nuclear. Nesse caso, a maneira de contornar tal inconsistência é apresentada na seção 2.4 .3 abaixo.

\subsubsection{A Interação de Contato de Fermi}

O fato do campo dipolar (2.12) ser indefinido na origem $(r=0)$ resulta em uma discrepância com relação ao resultado da integração (2.18). Como visto na seção 
2.4.2, a integral angular resulta em zero como solução, enquanto a parte radial tornase infinita mediante a integração.

Para encontrar o campo magnético associado ao spin nuclear numa região próxima ao centro do átomo, consideremos um núcleo com uma configuração arbitrária de correntes estacionárias, limitadas a uma região esférica de raio $R$. Em particular, nos passos seguintes, calcularemos o campo magnético clássico associado a esta distribuição de correntes, e usaremos o valor obtido no limite de $R \rightarrow 0$ como estimativa do campo magnético nuclear próximo ao centro atômico.

O campo magnético médio no interior do núcleo é, por definição, dado por:

$$
\boldsymbol{B}_{\text {medio }}=\frac{1}{\tau} \int \boldsymbol{B} d \tau^{\prime}
$$

Onde $\tau=\frac{4 \pi}{3} R^{3}$ (volume da esfera) e $d \tau^{\prime}$ o elemento infinitesimal de volume. Escrevendo-se $\boldsymbol{B}$ em termos do potencial vetor $\boldsymbol{A}$ e usando-se da identidade matemática algébrica ${ }^{6}$

$$
\int_{\text {Volume }}(\boldsymbol{\nabla} \times \boldsymbol{A}) d \tau^{\prime}=-\oint_{\text {Superficie }} \boldsymbol{A} \times d \boldsymbol{a}
$$

Segue que

$$
\boldsymbol{B}_{\text {medio }}=-\frac{1}{\tau} \int \boldsymbol{A} \times d \boldsymbol{a}
$$

Onde $d \boldsymbol{a}$ corresponde ao elemento infinitesimal de área na superfície da esfera, apontando na direção radial da mesma. Agora, como o potencial vetor $\boldsymbol{A}$ por si só consiste de uma integral sobre uma distribuição de correntes [14], segue que:

$$
\boldsymbol{A}=\frac{\mu_{0}}{4 \pi} \int \frac{\boldsymbol{J}\left(\boldsymbol{r}^{\prime}\right) d \tau^{\prime}}{\left|\boldsymbol{r}-\boldsymbol{r}^{\prime}\right|}
$$

Onde $\boldsymbol{r}^{\prime}$ define a distribuição das correntes na esfera e $\boldsymbol{r}$ define o ponto de observação na casca esférica $(|\boldsymbol{r}|=R)$, coforme a geometria apresentada na figura 2.4.

\footnotetext{
${ }^{6}$ A demonstração detalhada de tal relação encontra-se descrita no Apêndice B.
} 


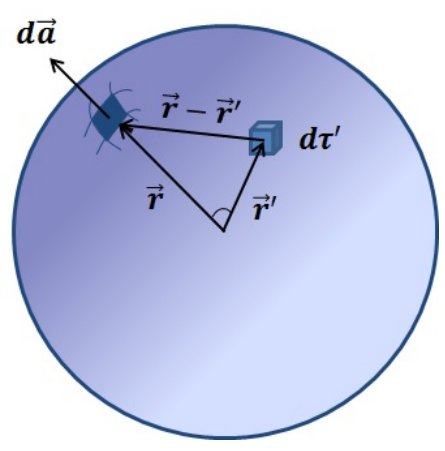

Figura 2.4: Geometria básica para equação (2.23), onde d $\boldsymbol{a}$ corresponde ao elemento infinitesimal de área na superfície da esfera; $d \tau^{\prime}$ corresponde ao elemento de volume interno à esfera; $\boldsymbol{r}^{\prime}$ define a distribuição de correntes na esfera e $\boldsymbol{r}$ define o ponto de observação. Adaptado de [17].

Consequentemente, segue que

$$
\begin{aligned}
\boldsymbol{B}_{\text {medio }} & =-\frac{1}{\tau} \frac{\mu_{0}}{4 \pi} \oint\left\{\int \frac{\boldsymbol{J}\left(\boldsymbol{r}^{\prime}\right) d \tau^{\prime}}{\left|\boldsymbol{r}-\boldsymbol{r}^{\prime}\right|}\right\} \times d \boldsymbol{a} \\
& =-\frac{1}{\tau} \frac{\mu_{0}}{4 \pi} \int \boldsymbol{J}\left(\boldsymbol{r}^{\prime}\right) \times\left\{\oint \frac{d \boldsymbol{a}}{\left|\boldsymbol{r}-\boldsymbol{r}^{\prime}\right|}\right\} d \tau^{\prime}
\end{aligned}
$$

uma vez que $\boldsymbol{J}$ depende apenas da localização das fontes (correntes) e, portanto, depende apenas de $\boldsymbol{r}^{\prime}$ e não da posição $\boldsymbol{r}$ de observação.

Para fazermos a integral de superfície, escolhemos primeiramente as coordenadas $(x, y, z)$ em termos das coordenadas esféricas $(r, \theta, \phi)$ e estabelecemos, sem perda de generalidade, o eixo polar junto a direção de $\boldsymbol{r}^{\prime}$. Assim, $\left|\boldsymbol{r}-\boldsymbol{r}^{\prime}\right|^{2}=R^{2}+z^{2}-2 R z^{\prime} \cos \theta$, enquanto que $d \boldsymbol{a}=R \operatorname{sen} \theta d \theta d \phi \hat{\boldsymbol{r}}$.

$$
\oint \frac{d \boldsymbol{a}}{\left|\boldsymbol{r}-\boldsymbol{r}^{\prime}\right|}=\oint \frac{(\cos \theta \hat{\boldsymbol{z}}+\operatorname{sen} \theta \cos \phi \hat{\boldsymbol{x}}+\operatorname{sen} \theta \operatorname{sen} \phi \hat{\boldsymbol{y}}) R^{2} \operatorname{sen} \theta d \theta d \phi}{\sqrt{R^{2}+z^{\prime 2}-2 R z^{\prime} \cos \theta}}
$$

As componentes $x$ e $y$ anulam-se mediante a integração em $\phi$, restando somente aquela ao longo da direção $\hat{z}$

$$
\oint \frac{d \boldsymbol{a}}{\left|\boldsymbol{r}-\boldsymbol{r}^{\prime}\right|}=2 \pi R^{2} \hat{\boldsymbol{z}} \int \frac{\operatorname{sen} \theta \cos \theta d \theta}{\sqrt{R^{2}+z^{\prime 2}-2 R z^{\prime} \cos \theta}}
$$

Por substituição da forma $u=\cos \theta \Rightarrow d u=-\operatorname{sen} \theta d \theta$ em (2.26)

$$
=2 \pi R^{2} \hat{z} \int \frac{u d u}{\sqrt{R^{2}+z^{\prime 2}-2 R z^{\prime} u}}
$$


Novamente por uma nova substituição $v=R^{2}+z^{\prime 2}-2 R z^{\prime} u \Rightarrow d v=-2 R z^{\prime} d u$ concluímos que

$$
\begin{gathered}
=2 \pi R^{2} \hat{\boldsymbol{z}}\left\{\frac{1}{\left(2 R z^{\prime}\right)^{2}} \int \frac{\left(v-R^{2}-z^{\prime 2}\right) d v}{\sqrt{v}}\right\} \\
=2 \pi R^{2} \hat{\boldsymbol{z}}\left\{\frac{1}{\left(2 R z^{\prime}\right)^{2}}\left[\frac{2}{3} v^{3 / 2}-2\left(R^{2}+z^{\prime 2}\right) v^{1 / 2}\right]\right\}=2 \pi R^{2} \hat{\boldsymbol{z}} \frac{1}{\left(2 R z^{\prime}\right)^{2}}\left\{\frac{2}{3} v^{1 / 2}\left[v-3\left(R^{2}+z^{\prime 2}\right)\right]\right\} \\
=2 \pi R^{2} \hat{\boldsymbol{z}} \frac{1}{\left(2 R z^{\prime}\right)^{2}}\left\{-\frac{2}{3}\left[2\left(R^{2}+z^{\prime 2}\right)+2 R z^{\prime} u\right] \sqrt{R^{2}+z^{\prime 2}-2 R z^{\prime} u}\right\}
\end{gathered}
$$

Aplicando-se os limites de integração, obtemos que:

$$
\begin{gathered}
=-\frac{2 \pi R^{2}}{3\left(R z^{\prime}\right)^{2}} \hat{z}\left\{\left[R^{2}+z^{\prime 2}+R z^{\prime}\right] \sqrt{R^{2}+z^{\prime 2}-2 R z^{\prime}}-\left[R^{2}+z^{\prime 2}-R z^{\prime}\right] \sqrt{R^{2}+z^{\prime 2}+2 R z^{\prime}}\right\} \\
=-\frac{2 \pi}{3 z^{\prime 2}} \hat{z}\left\{\left(R^{2}+z^{\prime 2}+R z^{\prime}\right)\left|R-z^{\prime}\right|-\left(R^{2}+z^{\prime 2}-R z^{\prime}\right)\left(R+z^{\prime}\right)\right\}
\end{gathered}
$$

Se $r^{\prime}<R$, ou seja, z' $<\mathrm{R}$, segue que

$$
\begin{aligned}
\oint \frac{d \boldsymbol{a}}{\left|\boldsymbol{r}-\boldsymbol{r}^{\prime}\right|} & =-\frac{2 \pi}{3 z^{\prime 2}} \hat{\boldsymbol{z}}\left[\left(R^{2}+z^{\prime 2}+R z^{\prime}\right)\left(R-z^{\prime}\right)-\left(R^{2}+z^{\prime 2}-R z^{\prime}\right)\left(R+z^{\prime}\right)\right] \\
& =-\frac{2 \pi}{3 z^{\prime 2}} \hat{\boldsymbol{z}}\left[-2 R^{2} z^{\prime}-2 z^{\prime 3}+2 R^{2} z^{\prime}\right]=\frac{4 \pi}{3} z^{\prime} \hat{\boldsymbol{z}}=\frac{4 \pi}{3} \boldsymbol{r}^{\prime}
\end{aligned}
$$

Finalmente, tomando-se esse resultado e aplicando-o na integração volumétrica de (2.24) concluímos que

$$
\boldsymbol{B}_{\text {medio }}=-\frac{3 \mu_{0}}{(4 \pi)^{2} R^{3}} \frac{4 \pi}{3} \int \boldsymbol{J}\left(\boldsymbol{r}^{\prime}\right) \times \boldsymbol{r}^{\prime} d \tau^{\prime}=\frac{\mu_{0}}{4 \pi} \frac{2 \boldsymbol{\mu}}{R^{3}}
$$

Onde $\boldsymbol{\mu}=\frac{1}{2} \int \boldsymbol{r}^{\prime} \times \boldsymbol{J}\left(\boldsymbol{r}^{\prime}\right) d \tau^{\prime}$ é o momento de dipolo total da esfera ${ }^{7}$.

O campo fora de tal esfera é dado precisamente pela equação (2.12), ou seja,

$$
\boldsymbol{B}_{\text {fora }}(\boldsymbol{r})=\frac{\mu_{0}}{4 \pi} \frac{1}{r^{3}}[3(\boldsymbol{\mu} \cdot \hat{\boldsymbol{r}}) \hat{\boldsymbol{r}}-\boldsymbol{\mu}] \quad \operatorname{para} r>R
$$

\footnotetext{
${ }^{7}$ Por exemplo, se considerarmos uma corrente $I$ através de uma espira de raio $R$, e definirmos a origem do sistema de coordenadas coincidente com o centro da espira, temos, em coordenadas cilíndricas, que a relação enunciada se reduz a $\boldsymbol{\mu}=1 / 2 \int \boldsymbol{r}^{\prime} \times\left(I d \boldsymbol{l}^{\prime}\right.$ ) (caso unidimensional ao longo de um fio $\left.\boldsymbol{J}\left(\boldsymbol{r}^{\prime}\right) d \tau^{\prime} \rightarrow I d \boldsymbol{l}^{\prime}\right)$, onde $\boldsymbol{r}^{\prime}=R \hat{\boldsymbol{r}}$ e $d \boldsymbol{l}^{\prime}=R d \theta \hat{\boldsymbol{\theta}}$. Isso implica em $\boldsymbol{\mu}=\frac{1}{2} \int(R \hat{\boldsymbol{r}}) \times(I R d \theta \hat{\boldsymbol{\theta}})=I\left(\pi R^{2}\right) \hat{\boldsymbol{z}}=I A \hat{\boldsymbol{z}}$, sendo A a área interna à espira. Tal exemplificação permite uma melhor compreensão da relação disposta, já que concorda com o resultado habitual de $\mu=I A$.
} 
Enquanto que o campo no interior da esfera é uniforme e dado por (2.31):

$$
\boldsymbol{B}_{\text {dentro }}(\boldsymbol{r})=\frac{\mu_{0}}{2 \pi} \frac{\boldsymbol{\mu}}{R^{3}} \quad \text { para } r<R
$$

No limite de um dipolo ideal $(R \rightarrow 0)$, a região interna a esfera contrai até zero, mas o campo magnético em si tende a infinito, de forma que a integral de $\boldsymbol{B}_{\text {dentro }}$ sobre a superfície esférica

$$
\int \boldsymbol{B}_{\text {dentro }} d \tau=\left(\frac{\mu_{0}}{2 \pi} \frac{\boldsymbol{\mu}}{R^{3}}\right)\left(\frac{4}{3} \pi R^{3}\right)=\frac{2}{3} \mu_{0} \boldsymbol{\mu}
$$

permaneça constante, não importando o quão pequeno o raio se torne. Quando $R \rightarrow 0$, portanto, o campo no interior da esfera pode ser descrito através de uma função delta de Dirac, na forma:

$$
\boldsymbol{B}_{\text {dentro }}(\boldsymbol{r})=\frac{2}{3} \mu_{0} \boldsymbol{\mu} \delta^{3}(\boldsymbol{r})
$$

tal termo é denominado Interação de Contato de Fermi e deve ser adicionado a (2.12) a fim de corrigir a indefinição do campo magnético na posição nuclear.

Portanto, o campo magnético de um dipolo ideal pode ser escrito como

$$
\boldsymbol{B}(\boldsymbol{r})=\frac{\mu_{o}}{4 \pi} \frac{1}{r^{3}}[3(\boldsymbol{\mu} \cdot \hat{\boldsymbol{r}}) \hat{\boldsymbol{r}}-\boldsymbol{\mu}]+\frac{2}{3} \mu_{0} \boldsymbol{\mu} \delta^{3}(\boldsymbol{r})
$$

com a compreensão de que o primeiro termo se aplica apenas a uma região externa a uma esfera infinitesimal centrada na origem.

Logo, a hamiltoniana de interação em um sistema elétron-núcleo pode ser descrita como:

$$
\begin{gathered}
\mathcal{H}=-\boldsymbol{\mu}_{\boldsymbol{e}} \cdot \boldsymbol{B}_{\boldsymbol{n}} \\
\mathcal{H}=\frac{\mu_{0}}{4 \pi} \frac{1}{r^{3}}\left[\boldsymbol{\mu}_{\boldsymbol{e}} \cdot \boldsymbol{\mu}_{\boldsymbol{n}}-3\left(\boldsymbol{\mu}_{\boldsymbol{e}} \cdot \hat{\boldsymbol{r}}\right)\left(\boldsymbol{\mu}_{\boldsymbol{n}} \cdot \hat{\boldsymbol{r}}\right)\right]-\frac{2}{3} \mu_{0} \boldsymbol{\mu}_{\boldsymbol{e}} \cdot \boldsymbol{\mu}_{\boldsymbol{n}} \delta^{3}(\boldsymbol{r})
\end{gathered}
$$




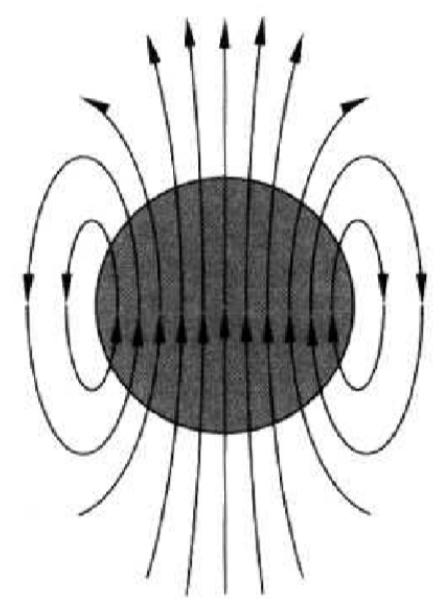

Figura 2.5: Diagrama esquemático da distribuição de campo magnético dentro e fora de um núcleo magnético (assumido esférico e sombreado no diagrama). A distribuição de campo magnético é causada por uma corrente que flui ao redor do equador da esfera mostrada em sombreado na figura. Fora da esfera, o campo magnético é devido a um dipolo puntiforme localizado no centro da esfera e cujo valor médio sobre um volume esfericamente simétrico resulta em zero. Dentro do núcleo, o campo está em média apontando para cima, de forma que o valor médio sobre um volume esfericamente simétrico não resulta em zero. Esta é a origem do termo de Interação de Contato de Fermi [18].

\subsubsection{A Interação Hiperfina de Contato de Fermi em Ilhas Quânticas}

Em contraste a um átomo convencional, um elétron confinado a uma ilha quântica interage com muitos sítios nucleares. Desta maneira, a hamiltoniana que descreve a Interação de Contato de Fermi - com todos os núcleos da rede - corresponde a uma contribuição conjunta da interação do spin eletrônico para com cada spin nuclear do sistema, ou seja,

$$
\mathcal{H}_{h f}=\frac{2 \mu_{0}}{3} g_{e} g_{n} \mu_{n} \mu_{B} \sum_{i} \boldsymbol{S} \cdot \boldsymbol{I}_{i} \delta^{3}\left(\boldsymbol{r}-\boldsymbol{r}_{i}\right)
$$

onde $\mu_{B}$ é o magneton de Bohr; $\mu_{n}$ é o magneton nuclear; $\boldsymbol{S}$ e $\boldsymbol{r}$ representam, respectivamente, o operador de spin e posição do elétron; $\boldsymbol{I}_{\boldsymbol{i}}$ e $\boldsymbol{r}_{\boldsymbol{i}}$ são os operadores de spin e posição associados ao i-ésimo núcleo do sistema; $g_{e}$ e $g_{n}$ são, nessa ordem, os fatores giromagnéticos eletrônico e nuclear [19]. 
O caso de maior relevância a ser abordado diz respeito a considerar um elétron no estado fundamental $\phi_{0}(\boldsymbol{r})$ de uma ilha quântica, cujo espaçamento entre os níveis de energia orbitais é muito maior que a escala típica de energia hiperfina, nos permitindo, portanto, o uso de teoria de perturbação. Nesta situação,

$$
\mathcal{H}_{I Q h f}=\left\langle\phi_{0}\left|\mathcal{H}_{h f}\right| \phi_{0}\right\rangle=\frac{4 \mu_{0}}{3} g_{n} \mu_{n} \mu_{B} \sum_{i}\left|\phi_{0}\left(\boldsymbol{r}_{\boldsymbol{i}}\right)\right|^{2} \boldsymbol{S} \cdot \boldsymbol{I}_{\boldsymbol{i}}
$$

no qual empregamos o valor de $g_{e}=2$ na equação (2.38).

A função de onda eletrônica no estado fundamental $\phi_{0}(\boldsymbol{r})$ pode ser escrita como a composição do produto de uma função de Bloch em $\boldsymbol{k}=0$, de amplitude $u_{0}$, por uma função envelope normalizada $\mathcal{F}(\boldsymbol{r})$, isto é, $\phi_{0}\left(\boldsymbol{r}_{\boldsymbol{i}}\right)=u_{0}\left(\boldsymbol{r}_{\boldsymbol{i}}\right) \mathcal{F}\left(\boldsymbol{r}_{\boldsymbol{i}}\right)$. ${ }^{8}$

Desta maneira, a hamiltoniana hiperfina efetiva pode ser escrita na forma:

$$
\mathcal{H}_{\text {IQhf }}=\boldsymbol{S} \cdot \boldsymbol{h}
$$

onde $\boldsymbol{h}=v_{0} \sum_{i} A_{i}\left|\mathcal{F}\left(\boldsymbol{r}_{\boldsymbol{i}}\right)\right|^{2} \boldsymbol{I}_{\boldsymbol{i}}$, sendo $A_{i}=\frac{4 \mu_{0}}{3} g_{n} \mu_{n} \mu_{B}\left|u_{0}\left(\boldsymbol{r}_{\boldsymbol{i}}\right)\right|^{2}$ e $v_{0}$ o volume da célula unitária cristalina.

O campo magnético hiperfino efetivo $\boldsymbol{B}_{\boldsymbol{n}}$, denominado Campo Overhauser, atuando sobre um spin eletrônico confinado a uma ilha quântica pode ser obtido de (2.40), tomando-se o valor esperado da hamiltoniana sobre o ensemble de funções de onda nucleares, isto é, a soma das contribuições de um grande número de núcleos.

$$
\begin{gathered}
\mu_{B} g_{e} \boldsymbol{S} \cdot \boldsymbol{B}_{\boldsymbol{n}}=\left\langle\mathcal{H}_{I Q h f}\right\rangle \\
\boldsymbol{B}_{\boldsymbol{n}}=\frac{v_{0}}{\mu_{B} g_{e}}\left\langle\sum_{i} A_{i}\left|\mathcal{F}\left(\boldsymbol{r}_{\boldsymbol{i}}\right)\right|^{2} \boldsymbol{I}_{\boldsymbol{i}}\right\rangle_{N}
\end{gathered}
$$

onde $\langle\ldots\rangle_{N}$ denota a média - em Mecânica Quântica - sobre o ensemble de funções de onda nucleares e $g_{e}$ é o fator giromagnético efetivo do elétron.

\footnotetext{
${ }^{8}$ Aqui assumimos as seguintes condições de normalização $\int_{v_{0}}\left|u\left(\boldsymbol{r}_{\boldsymbol{i}}\right)\right|^{2} d^{3} \boldsymbol{r}_{\boldsymbol{i}}=v_{0}$ e $\int\left|\mathcal{F}\left(\boldsymbol{r}_{\boldsymbol{i}}\right)\right|^{2} d^{3} \boldsymbol{r}_{\boldsymbol{i}}=$ 1, com entendimento de que a primeira integral é tomada sobre o volume $v_{0}$ da célula unitária, enquanto a segunda ocorre sobre o volume total do cristal.
} 
Para fins de quantificação, é interessante calcularmos os valores numéricos de campo efetivo em situações limites de polarização nuclear numa estrutura típica de GaAs. Por exemplo, do resultado (2.42), segue, de imediato, que na ausência de uma polarização nuclear ${ }^{9}$, o campo magnético efetivo $\boldsymbol{B}_{\boldsymbol{n}}$ é identicamente nulo, já que, nesse caso, a contribuição média dos spins nucleares em (2.42) é zero.

Entretanto, para a situação limite de total polarização dos núcleos atômicos, podemos estimar o campo efetivo máximo $\left(B_{n}^{\max }\right)$ em GaAs, ao considerarmos que, nessa estrutura: 1) Todos os spins nucleares são iguais (ou seja, $I_{i}=I=3 / 2$ ); 2) Em primeira ordem de aproximação, $\left.\left|\mathcal{F}\left(\boldsymbol{r}_{\boldsymbol{i}}\right)\right|^{2} \sim 1 / v_{0} ; \mathbf{3}\right)$ O fator giromagnético eletrônico efetivo é $\left.\left|g_{e}\right|=0,44[20] ; 4\right)$ A soma de $A_{i}$ sobre todos os núcleos em uma célula unitária é dada por $A=\sum_{i} A_{i} \approx 90 \mu \mathrm{eV}$ [21]. Desses resultados e da relação descrita em (2.42), temos que a estimativa para o campo efetivo máximo associado a saturação dos spins nucleares é da ordem de $B_{n}^{\max } \approx 5,3 \mathrm{~T}$ em GaAs.

\subsubsection{Defasagem do Spin Eletrônico devido às Flutuações do Campo Nuclear}

Do que já foi descrito anteriormente, temos que a polarização média do spin nuclear pode ser associada com um campo $\boldsymbol{B}_{\boldsymbol{n}}$, dito Campo Overhauser, atuando sobre um spin eletrônico. Para uma polarização nuclear claramente abaixo de 100\%, o campo nuclear flutua ao redor de um valor médio. A origem dessas flutuações estatísticas reside no fato de que há um número finito de núcleos internos à ilha quântica. Vários estudos teóricos previram que o mecanismo dominante no decaimento da polarização de spin em ilhas quânticas à baixas temperaturas, na ausência de campo magnético externo, é devido à interação para com essas flutuações de campo nuclear [22, 21].

Devemos notar, no entanto, que a frequência de precessão do eletron na presença do campo hiperfino nuclear é muito maior do que a frequência de precessão dos núcleos

\footnotetext{
${ }^{9} \mathrm{Ou}$ seja, na situação em que os spins dos núcleos apontam em direções completamente arbitrárias.
} 
devido à ação do campo hiperfino eletrônico ${ }^{10}$, ou seja, é como se a evolução temporal do spin do elétron observasse uma "imagem instantânea- uma configuração fixa - do campo nuclear. Nessa situação, cada spin eletrônico se move mediante uma flutuação congelada (do inglês, frozen fluctuation [10]) do campo magnético hiperfino nuclear $\boldsymbol{B}_{\boldsymbol{n}}$ - vide (2.42) - em sua própria ilha quântica. Esses campos, no entanto, estão aleatoriamente distribuídos dentre as ilhas do ensemble. Logo, mesmo que cada spin eletrônico precessione de uma forma coerente no campo hiperfino de sua própria ilha quântica, a polarização média de spin do ensemble decresce.

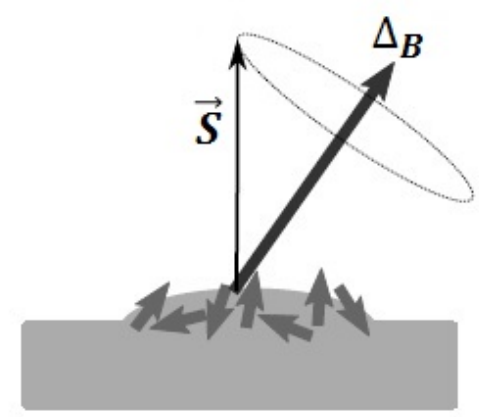

Figura 2.6: Ilustração da precessão do spin do elétron sobre as flutuações congeladas do campo hiperfino nuclear em um dado instante de tempo. $\Delta_{B}$ é construído mediante a soma sobre todos os spins nucleares individuais indicados pelas setas mais claras no interior do QD [23].

Em particular, a dispersão do campo nuclear em magnitude e direção, na ausência de uma dinâmica da polarização nuclear, é descrita por uma função de distribuição de probabilidades gaussiana em 3 dimensões [10, 21]

$$
\begin{gathered}
W\left(\boldsymbol{B}_{\boldsymbol{n}}\right)=W\left(B_{n x}\right) W\left(B_{n y}\right) W\left(B_{n z}\right) \\
W\left(\boldsymbol{B}_{\boldsymbol{n}}\right)=\frac{1}{\pi^{3 / 2} \Delta_{B}^{3}} \exp \left\{-\frac{B_{n x}^{2}}{\Delta_{B}^{2}}-\frac{B_{n y}^{2}}{\Delta_{B}^{2}}-\frac{B_{n z}^{2}}{\Delta_{B}^{2}}\right\}=\frac{1}{\pi^{3 / 2} \Delta_{B}^{3}} \exp \left\{-\frac{\boldsymbol{B}_{\boldsymbol{n}}^{2}}{\Delta_{B}^{2}}\right\}
\end{gathered}
$$

onde $\Delta_{B}$ é a dispersão da distribuição do campo hiperfino nuclear

$$
\Delta_{B}^{2}=2[\left\langle B_{n x}^{2}\right\rangle-\underbrace{\left\langle B_{n x}\right\rangle^{2}}_{=0}]=\frac{2}{3}\left\langle\boldsymbol{B}_{n}^{2}\right\rangle
$$

\footnotetext{
${ }^{10}$ Uma vez que os spins nucleares são isolados e não mudam sua orientação tão facilmente quanto os spins de origem eletrônica, o que pode ser interpretado como uma espécie de inércia dos núcleos ao movimento induzido pelo elétron.
} 
e consequentemente, $\Delta_{B}^{2}=\frac{2}{3} \sum_{i} I_{i}\left(I_{i}+1\right)\left(a_{i}\right)^{2}$, no qual $a_{i}=\frac{v_{0}}{\mu_{B} g_{e}} A_{i}\left|\mathcal{F}\left(\boldsymbol{r}_{\boldsymbol{i}}\right)\right|^{2}$ é o campo magnético de um único spin nuclear atuando sobre um elétron, assumindo-se as direções de spin nucleares como independentes uma das outras [21]. Em particular, no caso do GaAs, todos os spins nucleares são os mesmos, ou seja, $I_{i}=I=3 / 2$. Sendo assim, se substituirmos a soma sobre células unitárias por uma integração contínua em todo o espaço, obtemos que

$$
\Delta_{B}^{2}=\frac{2 I(I+1)}{3} \frac{\sum_{i}\left(A_{i}\right)^{2}}{\left(\mu_{B} g_{e}\right)^{2}} \frac{v_{0}}{V_{L}}=\frac{16 I(I+1)}{3 N_{L}} \frac{\sum_{i}\left(A_{i}\right)^{2}}{\left(\mu_{B} g_{e}\right)^{2}}
$$

onde a soma nesta equação se estende apenas sobre os núcleos compreendidos em uma célula unitária,

$$
V_{L}=\left(\int \psi^{4}(\boldsymbol{r}) d \tau\right)^{-1} \text { e } \quad N_{L}=\frac{8 V_{L}}{v_{0}}
$$

é o número de núcleos em um volume $V_{L}$, que efetivamente determinam a frequência de precessão do elétron. Particularmente, em GaAs, a somatória é aproximadamente $\Sigma_{i}\left(A_{i}\right)^{2} \approx 1.2 \times 10^{-3} \mathrm{meV}^{2}$ [21], o fator giromagnético eletrônico efetivo é $\left|g_{e}\right|=0,44$ [20] e o número típico de núcleos no interior do QD é da ordem de $N_{L}=10^{5}$ [21], o que resulta, portanto, em uma estimativa de $\Delta_{B} \simeq 20 \mathrm{mT}$ para a flutuação congelada do campo magnético nuclear.

\subsubsection{Defasagem de Spin Eletrônico na Flutuação Congelada do Campo Nuclear}

Vamos agora considerar um ensemble de ilhas quânticas, nas quais, através da incidência de luz circularmente polarizada, criamos (em $t=0)$ elétrons de mesma orientação de spin. Neste caso, os spins nucleares deste conjunto estão orientados aleatoriamente, de forma tal que os campos hiperfinos nas ilhas quânticas sejam distintos uns dos outros e, portanto, causem um efeito diferente na configuração inicial de spin do elétron $\boldsymbol{S}_{\mathbf{0}}$ em cada ilha. 
A precessão do spin $\boldsymbol{S}$ em um campo magnético fixo $\boldsymbol{B}$ pode ser formulada de uma maneira geral como:

$$
\boldsymbol{S}(t)=\left(\boldsymbol{S}_{\mathbf{0}} \cdot \boldsymbol{n}\right) \boldsymbol{n}+\left\{\boldsymbol{S}_{\mathbf{0}}-\left(\boldsymbol{S}_{\mathbf{0}} \cdot \boldsymbol{n}\right) \boldsymbol{n}\right\} \cos \omega t+\left[\left\{\boldsymbol{S}_{\mathbf{0}}-\left(\boldsymbol{S}_{\mathbf{0}} \cdot \boldsymbol{n}\right) \boldsymbol{n}\right\} \times \boldsymbol{n}\right] \operatorname{sen} \omega t
$$

onde $\boldsymbol{S}_{\mathbf{0}}$ é a configuração inicial de spin do elétron, $\boldsymbol{n}=\boldsymbol{B} / B$ é o vetor unitário na direção do campo magnético, e $\omega=\mu_{B} g_{e} B / \hbar$ é a frequência de precessão de Larmor do elétron ao redor do campo magnético [21], como representado na figura 2.7.

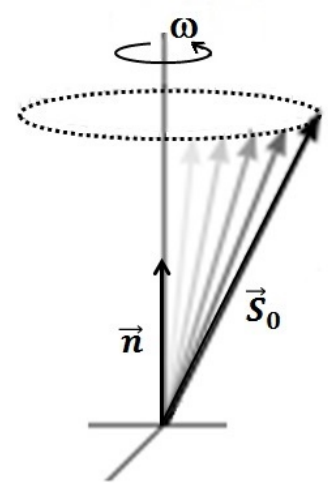

Figura 2.7: Representação esquemática da precessão do spin eletrônico inicial $\boldsymbol{S}_{\mathbf{0}}$ ao redor da direção $\boldsymbol{n}$;

A equação também descreve o movimento de precessão coerente do spin eletrônico em uma única ilha quântica devido ao campo magnético $\boldsymbol{B}_{\boldsymbol{n}}$ da flutuação congelada dos núcleos (onde $\boldsymbol{n}=\boldsymbol{B}_{\boldsymbol{n}} / B_{n}$ e $\left.\omega=\mu_{B} g_{e} B_{n} / \hbar\right)$. Calculando-se a média de (2.48) sobre a distribuição de campos magnéticos da equação (2.44), obtemos que a dependência temporal da polarização média dos spins eletrônicos de tal ensemble é descrita por:

$$
\langle\boldsymbol{S}(t)\rangle=\frac{\boldsymbol{S}_{\mathbf{0}}}{3}\left\{1+2\left[1-2\left(\frac{t}{T_{\Delta}}\right)^{2}\right] e^{-\left(t / T_{\Delta}\right)^{2}}\right\}
$$

Onde

$$
T_{\Delta}=\frac{\hbar}{\mu_{B} g_{e} \Delta_{B}}=\hbar \sqrt{\frac{3 N_{L}}{16 \sum_{i} I_{i}\left(I_{i}+1\right)\left(A_{i}\right)^{2}}}
$$

é o tempo de defasagem de spin do ensemble a campo nulo[21], proveniente das frequências de precessão eletrônica na flutuação congelada do campo hiperfino nuclear 
das ilhas quânticas. Em particular, esse tempo é da ordem de $T_{\Delta} \approx 1,4 \mathrm{~ns}$ em ilhas quânticas de GaAs, considerando-se a estimativa de $\Delta_{B} \simeq 20 \mathrm{mT}$ da seção anterior.

\subsection{Rotação de Faraday Resolvida no Tempo}

A construção de uma estrutura para dispositivos eletrônicos baseados na manipulação coerente de spin (denominada spintrônica) fornece uma grande motivação para o entendimento da evolução coerente de estados de spins em semicondutores [24, 25]. Neste caso, o objetivo fundamental é descobrir regimes nos quais estados quânticos de spin, cuidadosamente preparados, possam evoluir de forma coerente o bastante para viabilizar o armazenamento, manipulação e transporte de informação quântica.

Como resultado das regras de seleção de dipolo elétrico em semicondutores de gap direto, um fóton circularmente polarizado pode excitar um elétron de um estado de heavy hole de menor energia para o estado de mínima energia da banda de condução $[26,27]$. Desta maneira, se um campo magnético externo é aplicado perpendicular à direção de propagação do pulso circular, o elétron promovido pela interação passa a um estado de superposição coerente de estados (base) definidos pelo campo magnético. A evolução coerente dessa superposição resulta em uma diferença de fase entre os dois autoestados de energia envolvidos que varia linearmente no tempo como $\Delta E \cdot t / \hbar$. Uma forma semiclássica de se interpretar esse fenômeno diz respeito à precessão de Larmor do vetor de spin em um plano normal à direção do campo aplicado. Nesse modelo, a projeção do spin ao longo da direção inicial oscila como um cosseno, enquanto que, a evolução incoerente das autofunções de spin resulta em um decaimento da amplitude da oscilação. A "decoerência" de spin posterior pode se originar de diversos eventos possíveis, tais como espalhamento spin-spin devido à interação com buracos, impurezas magnéticas locais, espalhamento spin-órbita devido a impurezas ou fônons, ou efeitos de meia-vida finitos causados por recombinação com um buraco. Este último, 
em particular, faz parte do escopo de nosso estudo em questão.

Desta maneira, com o objetivo de obtermos informações a cerca da evolução temporal coerente de spin - que ocorre em escalas de tempo muito menores do que os tempos de recombinação radiativos - empregamos técnicas baseadas em medições dinâmicas do efeito Faraday, tal como a Rotação de Faraday Resolvida no Tempo (em inglês Time Resolved Faraday Rotation - TRFR).

As medidas se utilizando da técnica de TRFR são realizadas em uma geometria de transmissão nas quais dois feixes participantes - bombeio (pump) e sonda (probe) propagam-se ao longo da direção de um campo magnético externo aplicado ao sistema. Após a passagem pela amostra, mede-se o ângulo de rotação do pulso de sonda, em relação à sua direção original, em função do tempo de atraso entre os feixes pump-probe. Neste caso em particular, o pulso de bombeio é polarizado circularmente, enquanto que o de sonda/prova apresenta polarização linear.

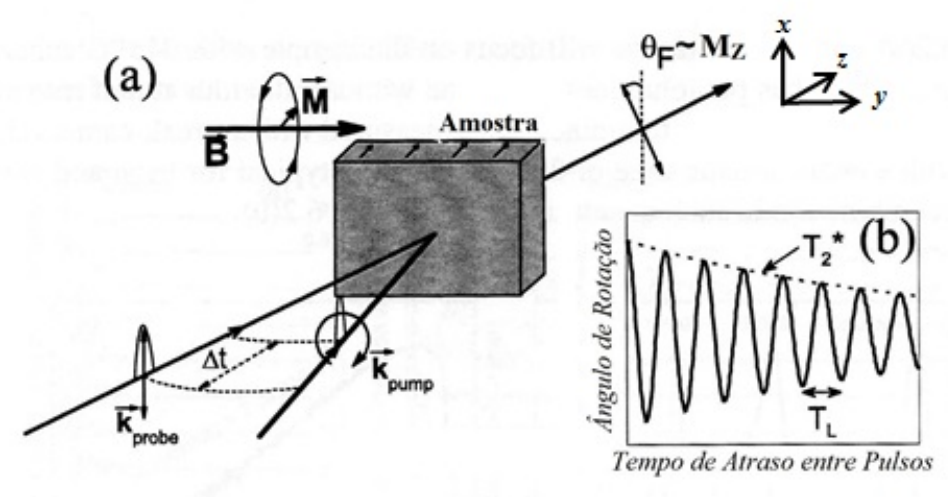

Figura 2.8: (a) Representação esquemática da técnica de rotação de Faraday resolvida no tempo; $\boldsymbol{B}$ é o campo magnético aplicado; $\boldsymbol{M}$ a magnetização; $\boldsymbol{k}_{\boldsymbol{p u m p}}$ o vetor de onda do feixe pump; $\boldsymbol{k}_{\text {probe }}$ o vetor de onda do probe e $\Delta t$ o atraso entre os pulsos. O ângulo de rotação $\theta_{F}$ é proporcional à componente $z$ da magnetização do conjunto de ilhas quânticas. (b) Sinal de TRFR devido a precessão de spin eletrônico ao redor do campo magnético transverso. $T_{L}=2 \pi / \Omega$ é o período de precessão do spin e $\Omega=g_{e} \frac{e B}{2 m_{0}}$ a frequência de precessão de Larmor. [28]

A figura 2.8, nos mostra um esquema simplificado da técnica TRFR em geometria Voigt. Nesse diagrama, as medidas de rotação de Faraday pump-probe são realizadas 
utilizando-se um pulso de bombeio, circularmente polarizado, com incidência normal ao plano no qual o campo magnético é aplicado. Nesse processo, o pulso de bombeio inicia o processo de transição de dipolo elétrico ao longo do gap do semicondutor, excitando, portanto, os pares de elétron-buraco que possuem spins polarizados na direção do caminho óptico, e os spins eletrônicos passam a precessionar ao redor do campo magnético a uma frequência característica denominada frequência de Larmor.

O resultado da rotação de Faraday $\left(\theta_{F}\right)$ é proporcional à oscilação da componente $z$ da magnetização $\left(M_{z}(t)\right)$ do conjunto de ilhas quânticas envolvidas e é bem descrita pela seguinte relação $[29,30,31]$

$$
M_{z}(t)=-M_{z 0} e^{-t / T_{2}^{*}} \cos (\Omega t+\phi)
$$

Onde $M_{z 0}$ é a amplitude da oscilação, $T_{2}^{*}$ é o tempo de defasagem de spin do conjunto de ilhas, $\Omega=g_{e} \frac{e B}{2 m_{0}}$ a frequência de Larmor, $g_{e}$ o fator giromagnético eletrônico e $\phi$ a fase da oscilação observada. Em particular, o sinal negativo na equação (2.51) refere-se a uma conveniência na definição da fase do sistema. Além disso, as magnitudes dos parâmetros $M_{z 0}$ e $\phi$ estão relacionadas ao mecanismo através do qual a luz gera magnetização no sistema de ilhas quânticas. 


\section{Capítulo 3}

\section{Material e Arranjo Experimental}

\subsection{Arranjo Experimental}

Devido ao longo tempo de vida dos estados de spin dos elétrons confinados, as ilhas quânticas carregadas com um único elétron são candidatas promissoras nas mais diversas aplicações em spintrônica e no desenvolvimento de dispositivos envolvendo processamento de informações quânticas.

De todos os métodos experimentais, o emprego de luz é o mais rápido possível na manipulação e leitura de estados confinados de spin, e portanto, é o que tem atraído maior atenção e enfoque por parte dos pesquisadores dessa área.

Um experimento padrão corresponde a iluminar uma amostra contendo um conjunto de ilhas quânticas carregadas usando-se um pulso de luz ultra-curto ressonante com o gap fundamental de energia das ilhas envolvidas, para em seguida, medirmos através da técnica de pump-probe - a magnetização do conjunto em função do tempo transcorrido [29, 32]. A esse método experimental dá-se o nome de Rotação de Faraday Resolvida no Tempo, descrita na seção 2.5 anterior.

Além disso, o processo de caracterização do conjunto de ilhas quânticas nas amostras se dá por meio da realização de medidas de fotoluminescência (em inglês, photoluminescence - PL). Tal técnica experimental é baseada no processo de recombinação dos elétrons fotoexcitados em semicondutores. Nesse modelo, os elétrons excitados 
por estímulo luminoso se recombinam com os buracos da banda de valência depois de certo tempo característico, e no instante de recombinação emitem um fóton cuja energia equivale a diferença energética dos estados quânticos envolvidos.

A seguir são especificados os sistemas utilizados para as medidas de Fotoluminescência e Rotação de Faraday empregados nesse trabalho.

\subsubsection{Montagem para Fotoluminescência}

O processo de fotoluminescência da amostra é realizado dispondo essa última em um criostato de baixíssima temperatura resfriado por Hélio líquido (LHe). Primeiramente, uma fibra é disposta de forma a conectar o laser monocromático de TitânioSafira $(\lambda=532 \mathrm{~nm})$ ao porta-amostra, atuando, portanto, como estímulo luminoso para fotoexcitação eletrônica. Uma segunda fibra é conectada de forma a guiar o sinal luminescente da amostra até o espectrômetro equipado com um dispositivo de carga acoplada (CCD), que por fim realiza a medida PL e envia o resultado a CPU. Uma figura esquemática de tal montagem encontra-se representada na figura 3.1 abaixo.

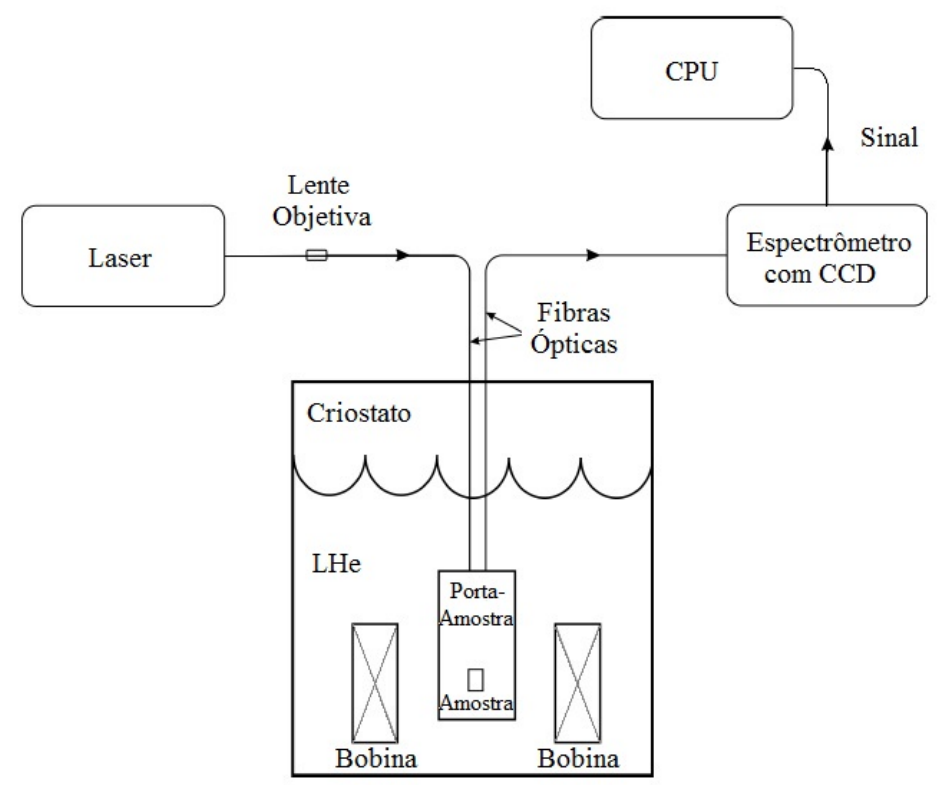

Figura 3.1: Arranjo experimental para medidas de fotoluminescência. [33] 


\subsubsection{Montagem para Rotação de Faraday}

O efeito medido por meio da técnica de pump-probe é dito Efeito Faraday que corresponde à rotação do plano de polarização da luz após a passagem por um meio previamente magnetizado. Nessa técnica, as ilhas quânticas são excitadas por um pulso de bombeio (pump) de $15 \mathrm{~mW}$ e medidas por um pulso sonda (probe) de $5 \mathrm{~mW}$ a uma temperatura amostral fixa de $\mathrm{T}=6 \mathrm{~K}$.

Um laser ajustável de Titânio-Safira é usado tanto no processo de excitação quanto na medição dos sinais provenientes das ilhas quânticas. Durante as medidas realizadas o laser é mantido no modo mode-locking emitindo pulsos de $1 \sim 2$ ps. Os pulsos são emitidos a uma frequência de 75,6 MHz. O feixe é dirigido a um beam splitter para geração dos feixes pump e probe. O feixe pump é atrasado em relação ao probe usando um espelho retrorrefletor, conforme apresentado na figura 3.2. A variação dos tempos de atraso entre os dois feixes é feita através da montagem do espelho retrorrefletor em uma delay line (ou linha de atraso). Finalmente, os feixes são focados na amostra presente no criostato de baixíssima temperatura, como esquematizado na figura 3.2.

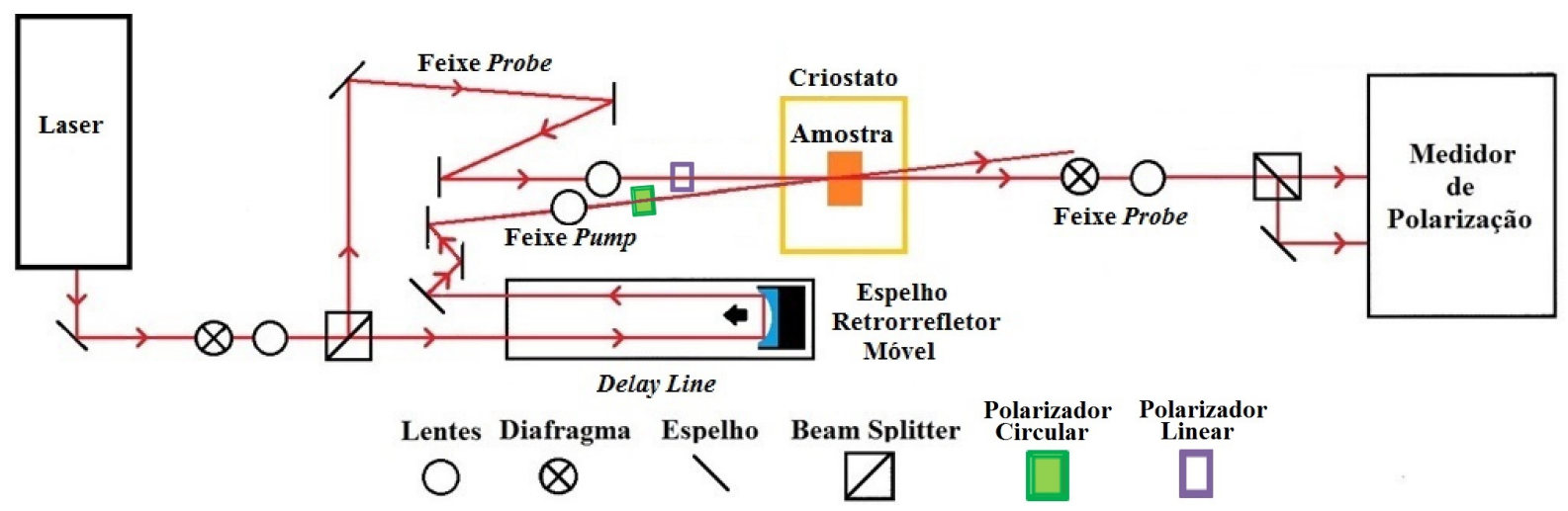

Figura 3.2: Figura esquemática do arranjo experimental empregado nas medições de rotação de Faraday resolvida no tempo (TRFR). O atraso (delay) entre os feixes participantes é realizado mediante o deslocamento do espelho retrorrefletor para esquerda, como indicado na ilustração. O feixe pump é disposto numa angulação tal que apenas o feixe probe atinja o medidor de polarização. Adaptado de [34] 


\subsection{Amostra}

A estrutura investigada nesse trabalho corresponde a uma amostra contendo ilhas quânticas carregadas de (In, Ga)As/GaAs com ocupação média de um elétron por QD. A amostra foi crescida por meio da técnica de Molecular Beam Epitaxy (MBE) na direção [100] do substrato de GaAs.

A amostra contendo 10 camadas de ilhas quânticas de GaAs carregadas negativamente foi termicamente tratada por 30s à temperatura de $950^{\circ} \mathrm{C}$, de forma a exibir uma emissão fotoluminescente ao redor de $1,35 \mathrm{eV}(\lambda \approx 915 \mathrm{~nm})$, conforme apresentado na figura 3.3 a seguir. A amostra foi investigada usando um criostato magneto-óptico de temperatura fixa em $T=6 K$.

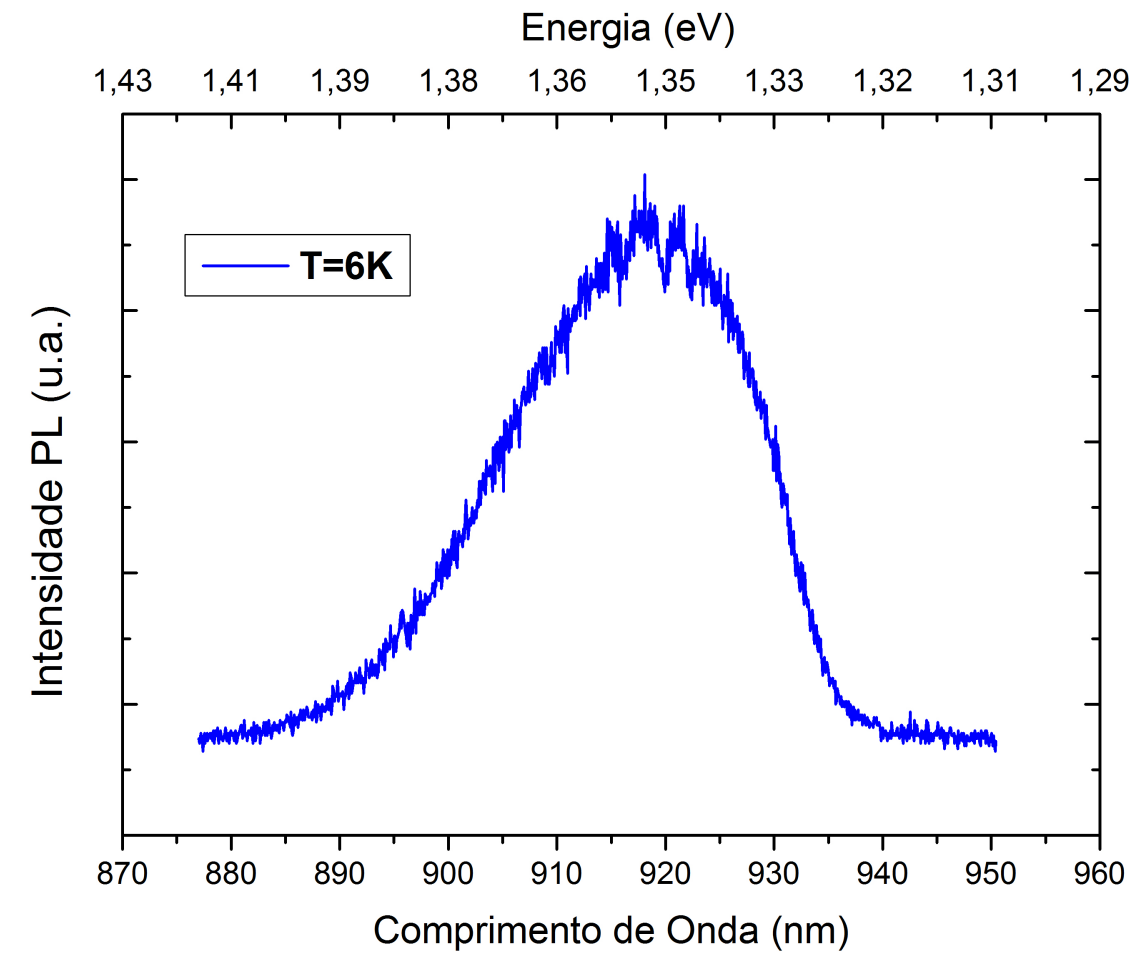

Figura 3.3: Espectro fotoluminescente à temperatura fixa de $T=6 K$ da amostra empregada.

O sinal de TRFR detectado nas medidas realizadas está correlacionado a uma excitação simultânea de um grande número de ilhas quânticas. Estima-se que esse 
número de QD's contribuintes ao sinal seja da ordem de algumas centenas de milhares de ilhas [30].

A curva mostrada na figura 3.3 corresponde ao espectro de fotoluminescência da amostra empregada. O espectro total do conjunto de ilhas quânticas observado consiste de uma superposição de linhas estreitas de PL associadas aos QD's iluminados pelo feixe laser. Desta maneira, a medida do espectro PL atua como um fator indicativo na distribuição de tamanhos das ilhas quânticas envolvidas.

O espectro PL do conjunto de QD's apresenta uma forma Lorentziana, na qual a energia de máxima intensidade denota a energia de transição mais presente na amostra, ao mesmo tempo que a largura à meia altura reflete a dispersão em energias e corresponde a uma medida da dispersão de tamanhos das ilhas quânticas iluminadas. 


\section{Capítulo 4}

\section{Resultados e Análises}

\subsection{Orientação Óptica de Spin em Semicondutores}

A coerência de spins em ilhas quânticas pode ser gerada pela interação de fótons ressonantes com a energia de transição dos QD's envolvidos, a esse fenômeno dá-se o nome de Orientação Óptica de Coerência de Spins.

Na seção que segue, o modelo vigente na descrição do processo de geração de coerência é descrito, qualitativamente, usando-se de um cenário introdutório para compreensão do conceito.

As demais seções desenvolvem uma descrição quantitativa do processo óptico de orientação de spins confinados às ilhas quânticas tanto para o modelo atualmente aceito quanto para o o modelo proposto.

\subsection{Descrição Qualitativa para Geração de Coerência de Spin}

Nos estados estacionários, os spins em diferentes ilhas quânticas do ensemble (ou conjunto) não se encontram correlacionados, ou seja, não apontam numa direção específica do espaço, o que resulta em uma magnetização total nula para o sistema. Para um ensemble de QD's, cada qual contendo um único elétron residente em seu interior, o cenário aceito na descrição do processo de geração de coerência de spin é entendido 
como segue:

Anteriormente a chegada do pulso de bombeio, o estado de spin do elétron residente em uma dada ilha quântica corresponde à superposição dos estados spin para cima e para baixo ${ }^{1}$, ou seja,

$$
\Psi(t<0)=\alpha|\uparrow\rangle+\beta|\downarrow\rangle, \text { onde }|\alpha|^{2}+|\beta|^{2}=1
$$

Devido às regras de seleção óptica, um pulso de luz circularmente polarizado para a direita (para a esquerda) acopla a banda de valência à componente de spin para cima (spin para baixo) da função de onda inicial (4.1). Logo, a absorção do fóton no instante de tempo $t=0$ altera a função de onda para ${ }^{2}$

$$
\Psi(t=0)=\alpha|\uparrow \downarrow \uparrow\rangle+\beta|\downarrow\rangle
$$

Na primeira componente de (4.2), os spins eletrônicos encontram-se acoplados e um estado de spin singleto - e portanto, de spin total nulo - que não precessiona mediante a aplicação de um campo magnético externo. No entanto, a segunda componente de (4.2), constituída pelo spin eletrônico isolado, precessiona ao redor desse campo e dá origem a um sinal oscilante no tempo.

Se assumirmos que no instante de recombinação do tríon, $t=t_{r}$, o buraco captura com igual probabilidade qualquer um dos dois elétrons participantes da primeira componente da função de onda [35, 36, 37], obtemos uma contribuição média da forma:

$$
\left.\left.\Psi\left(t>t_{r}\right)=\alpha \mid \text { Spin Aleatório }\right\rangle+\beta \mid \text { Spin Precessionante }\right\rangle
$$

Por consequência, segundo essa consideração, para $t>t_{r}$, a magnetização não-nula está conectada à componente de spin não emparelhada e não afetada pela excitação do pulso de luz. Além disso, o modelo prevê uma única fase de precessão de spin

\footnotetext{
${ }^{1}$ Definidos em relação ao vetor de onda do fóton incidente. Por simplicidade, essa direção é dada como o eixo $z$ do sistema;

2 个 denota o spin do buraco no exciton;
} 
constante, bem como uma amplitude de oscilação fixa e independente da intensidade do campo magnético aplicado.

No entanto, tal previsão do modelo encontra-se em discordância direta com os resultados experimentais, nos quais a fase indica ser dependente da energia do fóton e da intensidade do campo magnético, enquanto a amplitude indica ser variável com a intensidade do campo externo aplicado [35]. Sendo assim, visando a descrição de tais comportamentos experimentais, relatamos nas seções seguintes o desenvolvimento de um modelo teórico quantitativo de indução magnética pela luz levando-se em consideração o movimento de precessão do buraco constituinte do tríon.

\subsection{Modelo Vigente na Descrição de Coerência de Spin para Transições $e_{s} \rightarrow S_{s}$ e $e_{s} \rightarrow S_{p}$}

Particularmente, se considerarmos uma ilha quântica carregada com um único elétron submetida a um campo magnético ao longo do eixo $y$, segue que a hamiltoniana associada ao spin eletrônico é descrita como uma interação Zeeman e expressa por ${ }^{3}$ :

$$
\mathcal{H}=g \mu_{B} B \frac{S_{y}}{\hbar}=\frac{1}{2} g \mu_{B} B \sigma_{y}=\frac{1}{2} \hbar \Omega \sigma_{y}
$$

na qual $\sigma_{y}$ é a matriz de spin de Pauli, $\Omega=\frac{g \mu_{B} B}{\hbar}$ é a frequência de precessão de Larmor $^{4}$ e $\mu_{B}=\frac{e \hbar}{2 m_{0}}$ é o magneton de Bohr.

Figura 4.1: Figura esquemática do sistema de eixos referenciais adotados.

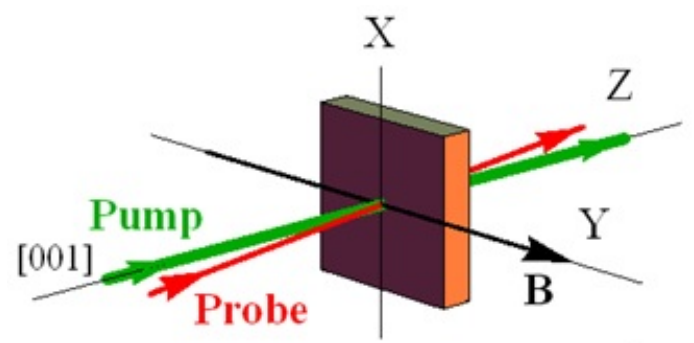

\footnotetext{
${ }^{3}$ Aqui estamos ignorando o campo Overhauser, dado pela equação (2.42), pois segundo a estimativa realizada na seção 2.4.5, o mesmo apresenta magnitude de algumas dezenas de $m T$ que é muito menor do que os campos empregados experimentalmente e que consideraremos no decorrer desta seção.

${ }^{4}$ Segundo a referência [38], sabe-se que, em ilhas de (In,Ga)As/GaAs, $g<0$, no entanto, por simplicidade e sem perda de generalidade, adotaremos nos passos seguintes $g>0$. Para os cálculos desta seção, o sinal do fator giromagnético não é relevante e não altera a solução física final.
} 
Desta maneira, os autoestados de $\mathcal{H}$ são os mesmos de $\sigma_{y}$, e podem ser obtidos por meio de:

$$
\left(\begin{array}{cc}
0 & -i \\
i & 0
\end{array}\right)\left(\begin{array}{l}
a \\
b
\end{array}\right)=\lambda\left(\begin{array}{l}
a \\
b
\end{array}\right) \Rightarrow \operatorname{det}\left(\begin{array}{cc}
-\lambda & -i \\
i & -\lambda
\end{array}\right)=0 \Rightarrow \lambda= \pm 1
$$

mediante a normalização $|a|^{2}+|b|^{2}=1$, segue que o conjunto de autovetores e autovalores correspondentes são dados por

$$
\chi_{+}^{(y)}=\frac{1}{\sqrt{2}}\left(\begin{array}{l}
1 \\
i
\end{array}\right) \text { de autovalor } \lambda=1 \quad \text { e } \quad \chi_{-}^{(y)}=\frac{1}{\sqrt{2}}\left(\begin{array}{c}
1 \\
-i
\end{array}\right) \text { de autovalor } \lambda=-1
$$

que podem ser reescritos em termos dos estados-base $|\uparrow\rangle$ e $|\downarrow\rangle$ apontando na mesma direção ("para cima") ou na direção oposta ("para baixo") ao eixo $z$ de polarização:

$$
\chi_{+}^{(y)}=\frac{1}{\sqrt{2}}\left(\begin{array}{l}
1 \\
i
\end{array}\right)=\frac{1}{\sqrt{2}}\left\{\left(\begin{array}{l}
1 \\
0
\end{array}\right)+i\left(\begin{array}{l}
0 \\
1
\end{array}\right)\right\}=\frac{1}{\sqrt{2}}\{|\uparrow\rangle+i|\downarrow\rangle\}=\frac{1}{\sqrt{2}}|\uparrow+i \downarrow\rangle
$$

Analogamente,

$$
\chi_{-}^{(y)}=\frac{1}{\sqrt{2}}\left(\begin{array}{c}
1 \\
-i
\end{array}\right)=\frac{1}{\sqrt{2}}\left\{\left(\begin{array}{l}
1 \\
0
\end{array}\right)-i\left(\begin{array}{l}
0 \\
1
\end{array}\right)\right\}=\frac{1}{\sqrt{2}}\{|\uparrow\rangle-i|\downarrow\rangle\}=\frac{1}{\sqrt{2}}|\uparrow-i \downarrow\rangle
$$

Desta forma, segue que os autoestados de $\mathcal{H}$ e respectivas energias associadas são dados por

$$
\left\{\begin{array}{l}
\chi_{+}^{(y)}, \text { com energia } E_{+}=\hbar \Omega / 2 \\
\chi_{-}^{(y)}, \text { com energia } E_{-}=-\hbar \Omega / 2
\end{array}\right.
$$

Se considerarmos que o sistema parte do equilíbrio (estado de mínima energia), temos que o spin eletrônico se inicia polarizado na direção - $\hat{y}$, e portanto, a função de spin da ilha quântica, em um dado instante anterior à chegada do pulso laser, é dada $\operatorname{por}^{5}$ :

$$
\Psi=\frac{1}{\sqrt{2}}|\uparrow-i \downarrow\rangle
$$

A evolução temporal do spin eletrônico - anterior à chegada do pulso - é determinada ao aplicarmos o operador $e^{-\frac{i t \mathcal{H}}{\hbar}}$ sobre a função de spin inicial (4.10).

$$
\Psi(t<0)=e^{-\frac{i \mathcal{H} \mathcal{H}}{\hbar}}\left\{\frac{1}{\sqrt{2}}|\uparrow-i \downarrow\rangle\right\}
$$

\footnotetext{
${ }^{5}$ Em particular, se $\mathrm{g}<0$ em (4.4), o estado de menor energia é dado por $\chi_{+}^{(y)}$, o que corresponde a uma separação energética $\Delta E=E_{+}-E_{-}=\hbar \Omega$;
} 
Para tanto, calculemos a seguinte expansão em série de potências

$$
e^{-\frac{i t \mathcal{H}}{\hbar}}=e^{-\frac{i}{2} \Omega t \sigma_{y}}=\sum_{n=0}^{\infty} \frac{\left(-\frac{i}{2} \Omega t \sigma_{y}\right)^{n}}{n !}
$$

Se levarmos em conta a propriedade de potenciação de $\sigma_{y}$ dada por

$$
\left(\sigma_{y}\right)^{n}= \begin{cases}\mathbb{I}, & \text { se } n \text { for par } \\ \sigma_{y}, & \text { se } n \text { for ímpar }\end{cases}
$$

onde I é a matriz identidade de ordem 2 .

Podemos retornar a (4.12) e dividir a soma em duas composições distintas

$$
\begin{aligned}
e^{-\frac{i t \mathcal{H}}{\hbar}} & =\sum_{n \text { par }} \frac{\left(-\frac{i}{2} \Omega t\right)^{n}}{n !}+\sigma_{y} \sum_{n \text { impar }} \frac{\left(-\frac{i}{2} \Omega t\right)^{n}}{n !} \\
& =\sum_{n=0}^{\infty}(-1)^{n} \frac{\left(\frac{\Omega t}{2}\right)^{2 n}}{2 n !}+\sigma_{y} \cdot(-i) \sum_{n=0}^{\infty}(-1)^{n} \frac{\left(\frac{\Omega t}{2}\right)^{2 n+1}}{(2 n+1) !} \\
& =\mathbb{I} \cos \frac{\Omega t}{2}-i \sigma_{y} \operatorname{sen} \frac{\Omega t}{2}
\end{aligned}
$$

Por consequência, concluímos que

$$
\begin{aligned}
e^{\frac{-i t \mathcal{H}}{\hbar}}|\uparrow\rangle & =\cos \frac{\Omega t}{2} \mathbb{I}|\uparrow\rangle-i \operatorname{sen} \frac{\Omega t}{2}\left(\begin{array}{cc}
0 & -i \\
i & 0
\end{array}\right)|\uparrow\rangle \\
& =\cos \frac{\Omega t}{2}|\uparrow\rangle-i \operatorname{sen} \frac{\Omega t}{2}\left(\begin{array}{cc}
0 & -i \\
i & 0
\end{array}\right)\left(\begin{array}{l}
1 \\
0
\end{array}\right) \\
& =\cos \frac{\Omega t}{2}|\uparrow\rangle+\operatorname{sen} \frac{\Omega t}{2}|\downarrow\rangle
\end{aligned}
$$

Analogamente,

$$
e^{\frac{-i t \mathcal{H}}{\hbar}}|\downarrow\rangle=\cos \frac{\Omega t}{2}|\downarrow\rangle-\operatorname{sen} \frac{\Omega t}{2}|\uparrow\rangle
$$

Finalmente, a partir desses resultados, podemos obter a evolução temporal da função de onda de spin através de (4.11)

$$
\begin{gathered}
\Psi(t<0)=e^{-\frac{i t \mathcal{H}}{\hbar}}\left\{\frac{1}{\sqrt{2}}|\uparrow-i \downarrow\rangle\right\} \\
=\frac{1}{\sqrt{2}}\left[\left(\cos \frac{\Omega t}{2}|\uparrow\rangle+\operatorname{sen} \frac{\Omega t}{2}|\downarrow\rangle\right)-i\left(\cos \frac{\Omega t}{2}|\downarrow\rangle-\operatorname{sen} \frac{\Omega t}{2}|\uparrow\rangle\right)\right]
\end{gathered}
$$


$$
=\frac{1}{\sqrt{2}}\left[\left(\cos \frac{\Omega t}{2}+i \operatorname{sen} \frac{\Omega t}{2}\right)|\uparrow\rangle-i\left(\cos \frac{\Omega t}{2}+i \operatorname{sen} \frac{\Omega t}{2}\right)|\downarrow\rangle\right]
$$

$$
\Psi(t<0)=e^{\frac{i \Omega t}{2}}\left\{\frac{1}{\sqrt{2}}|\uparrow-i \downarrow\rangle\right\}
$$

que corresponde a apenas uma mudança de fase (4.10), e portanto, não há precessão para instantes anteriores à chegada do pulso $(\mathrm{t}<0)$.

De forma a compreender como um elétron em uma dada ilha quântica pode ter seu spin orientado, partamos das seguintes considerações:

Seja o estado fundamental, $e_{s}$, de uma ilha quântica dado pela presença de um elétron residente numa camada $s$. O estado de menor energia do tríon fotoexcitado é composto por um elétron adicional na mesma camada $s$ e de spin oposto aquele do primeiro elétron, mais um buraco localizado na camada $s$ da banda de valência. Por simplicidade, vamos denotar essa configuração do tríon como $S_{s}$, para simbolizar o estado de spin total zero (S) - dito singleto - e o subescrito para representar a natureza tipo- $s$ das partículas fotoexcitadas. Vide figura 4.2.

A outra transição óptica permitida para o estado de spin do tríon é composta pela presença do elétron e do buraco fotoexcitado dispostos em suas respectivas camadas tipo- $p$; Tal tríon pode ser excitado com um spin eletrônico total zero ou um, correspondentes, respectivamente, aos estados singleto $\left(S_{p}\right)$ e tripleto $\left(T_{p}\right)$, conforme apresentado na figura 4.2 .

Suponhamos que no instante de tempo $\mathrm{t}=0$, um pulso laser, se propagando ao longo da direção $\hat{z}$ e ressonante com a transição $e_{s} \rightarrow S_{s}$, atinja uma ilha quântica e altere instantaneamente seu estado (4.17) para $^{6}$

$$
\Psi(t=0)=\frac{1}{\sqrt{2}}|\uparrow \downarrow \uparrow-i \downarrow\rangle
$$

\footnotetext{
${ }^{6}$ Esta mesma função de onda de spin pode ser aplicada a qualquer excitação singleto, a exemplo da transição $e_{s} \rightarrow S_{p}$.
} 
Figura 4.2: Esquema dos níveis de energia dos tríons $\left(S_{s}, T_{p}\right.$ e $\left.S_{p}\right)$ fotoexcitados provenientes da sintonização do laser de bombeio ( $p u m p$ ) - circularmente polarizado para direita - para com as transições envolvendo as camadas $s$ e $p$; As setas representam o acoplamento do estado fundamental - equivalente a uma ilha quântica de um único elétron, cuja função de spin é composta por uma componente para cima (esquerda) e outra para baixo (direita) - para com os estados excitados; As funções de spin dos elétrons associados aos estados do tríon são exibidas à direita. [35]

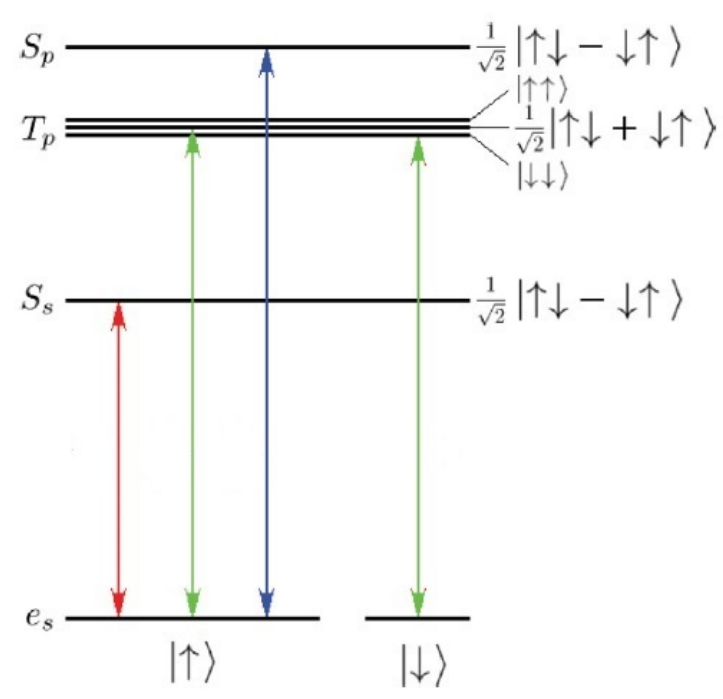

Com o passar do tempo, os elétrons emparelhados na primeira componente da função de spin (4.18) não precessionam, tendo em vista que a interação de exchange elétron-elétron faz com que tal componente permaneça num estado singleto com spin total zero. Por outro lado, tanto o buraco quanto o elétron da segunda componente da função de spin precessionam, já que nesses casos não há uma compensação como na primeira situação citada. Dessa maneira, a função de onda evolui no tempo, até o instante de recombinação $\left(t_{r}\right)$ do tríon, na forma

$$
\Psi\left(0<t<t_{r}\right)=\frac{1}{\sqrt{2}}\left|\uparrow \downarrow \chi_{h}-i \chi_{e}\right\rangle
$$

Onde $\chi_{h}$ é a função que descreve o movimento de precessão do spin do buraco, e $\chi_{e}$ a função de precessão do elétron. Vale notar, que podemos obter a função $\chi_{e}$ por meio da aplicação do operador de evolução temporal sobre a segunda componente de

$$
\chi_{e}=e^{\frac{-i t \mathcal{H}}{\hbar}}|\downarrow\rangle=|\cos (\Omega t / 2) \downarrow-\operatorname{sen}(\Omega t / 2) \uparrow\rangle
$$




\subsection{Modelo Vigente na Descrição de Coerência de Spin para Transições}

$$
\underline{e_{s} \rightarrow S_{s} \text { e } e_{s} \rightarrow S_{p}}
$$

Desta maneira, o momento magnético do $\mathrm{QD}$, no instante anterior à recombinação do tríon, é proporcional ao valor médio de $\boldsymbol{\sigma}=\sigma_{x} \hat{x}+\sigma_{y} \hat{y}+\sigma_{z} \hat{z}$ e encontra-se associado à componente de spin não-excitada da função de onda. Portanto, segue que

$$
\left\langle\boldsymbol{\sigma}\left(t<t_{r}\right)\right\rangle=\int \Psi^{*}\left(0<t<t_{r}\right) \boldsymbol{\sigma} \Psi\left(0<t<t_{r}\right) d \tau=\frac{1}{2} \int \chi_{e}^{*} \boldsymbol{\sigma} \chi_{e} d \tau
$$

Em particular, para a componente $x$ temos que ${ }^{7}$

$$
\begin{gathered}
\left\langle\sigma_{x}\right\rangle=\left\langle\chi_{e}\left|\sigma_{x}\right| \chi_{e}\right\rangle=\left\langle\chi_{e}\left|\sigma_{x}\right| \cos (\Omega t / 2) \downarrow-\operatorname{sen}(\Omega t / 2) \uparrow\right\rangle \\
=\left\langle\chi_{e} \mid \cos (\Omega t / 2) \uparrow-\operatorname{sen}(\Omega t / 2) \downarrow\right\rangle=-2 \operatorname{sen}(\Omega t / 2) \cos (\Omega t / 2) \\
\therefore\left\langle\sigma_{x}\right\rangle=-\operatorname{sen} \Omega t
\end{gathered}
$$

Ao efetuarmos o mesmo procedimento para as demais componentes, concluímos que

$$
\left\langle\boldsymbol{\sigma}\left(t<t_{r}\right)\right\rangle=-\hat{x} \frac{1}{2} \operatorname{sen} \Omega t-\hat{z} \frac{1}{2} \cos \Omega t
$$

que corresponde a um movimento de precessão causado pela componente de spin não emparelhada.

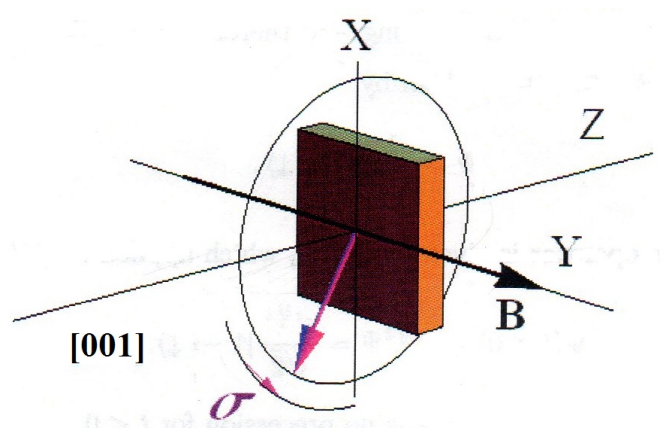

Figura 4.3: Precessão da magnetização devido à componente de spin nãoexcitada da função eletrônica.

No instante da recombinação do tríon, $t=t_{r}$ segue, das equações (4.18) e (4.20), que caso o buraco venha a capturar o elétron de spin para cima, a função de onda eletrônica obedece

$$
\Psi_{A}\left(t=t_{r}\right)=\frac{1}{\sqrt{2}}\left|\downarrow-i \cos \frac{\Omega}{2} t_{r} \downarrow+i \operatorname{sen} \frac{\Omega}{2} t_{r} \uparrow\right\rangle
$$

\footnotetext{
${ }^{7}$ Lembrando que $\sigma_{x}|\uparrow\rangle=|\downarrow\rangle$ e $\sigma_{x}|\downarrow\rangle=|\uparrow\rangle$
} 
Por outro lado, caso o buraco venha a capturar o elétron de spin apontando da direção oposta, a função de onda eletrônica segue

$$
\Psi_{B}\left(t=t_{r}\right)=\frac{1}{\sqrt{2}}\left|\uparrow-i \cos \frac{\Omega}{2} t_{r} \downarrow+i \operatorname{sen} \frac{\Omega}{2} t_{r} \uparrow\right\rangle
$$

Novamente, a evolução desses estados no tempo é determinada mediante a aplicação do operador de evolução temporal, ou seja,

$$
\Psi\left(t>t_{r}\right)=e^{-\frac{i\left(t-t_{r}\right)}{\hbar} \mathcal{H}} \Psi\left(t_{r}\right)
$$

Desta maneira, ao aplicarmos os resultados de (4.15) e (4.16) sobre $\Psi_{A}$, obtemos que

$$
\begin{aligned}
\Psi_{A}\left(t>t_{r}\right) & =\frac{1}{\sqrt{2}}\left\{\left(1-i \cos \frac{\Omega}{2} t_{r}\right)\left[\cos \frac{\Omega}{2}\left(t-t_{r}\right)|\downarrow\rangle-\operatorname{sen} \frac{\Omega}{2}\left(t-t_{r}\right)|\uparrow\rangle\right]+\right. \\
& \left.+i \operatorname{sen} \frac{\Omega}{2} t_{r}\left(\cos \frac{\Omega}{2}\left(t-t_{r}\right)|\uparrow\rangle+\operatorname{sen} \frac{\Omega}{2}\left(t-t_{r}\right)|\downarrow\rangle\right)\right\}
\end{aligned}
$$

ou ainda

$$
\Psi_{A}\left(t>t_{r}\right)=\frac{1}{\sqrt{2}}\left\{\left[i \operatorname{sen} \frac{\Omega}{2} t-\operatorname{sen} \frac{\Omega}{2}\left(t-t_{r}\right)\right]|\uparrow\rangle+\left[-i \cos \frac{\Omega}{2} t+\cos \frac{\Omega}{2}\left(t-t_{r}\right)\right]|\downarrow\rangle\right\}
$$

Analogamente, aplicando-se o mesmo procedimento a $\Psi_{B}$ :

$$
\Psi_{B}\left(t>t_{r}\right)=\frac{1}{\sqrt{2}}\left\{\left[i \operatorname{sen} \frac{\Omega}{2} t+\cos \frac{\Omega}{2}\left(t-t_{r}\right)\right]|\uparrow\rangle+\left[-i \cos \frac{\Omega}{2} t+\operatorname{sen} \frac{\Omega}{2}\left(t-t_{r}\right)\right]|\downarrow\rangle\right\}
$$

Para sabermos se esses resultados encontram-se relacionados à movimentos de precessão, precisamos calcular os valores médios de $\boldsymbol{\sigma}$ para cada um dos casos anteriores.

Assim, usando-se das matrizes de Pauli, o valor esperado do momento magnético para ilhas quânticas no estado A é dado por:

$$
\left\langle\boldsymbol{\sigma}_{A}\left(t>t_{r}\right)\right\rangle=-\hat{x} \operatorname{sen}\left(\Omega t-\frac{\Omega}{2} t_{r}\right) \cos \frac{\Omega}{2} t_{r}-\hat{y} \operatorname{sen} \frac{\Omega}{2} t_{r}-\hat{z} \cos \left(\Omega t-\frac{\Omega}{2} t_{r}\right) \cos \frac{\Omega}{2} t_{r}
$$


Enquanto que para ilhas quânticas no estado B é dado por:

$$
\left\langle\boldsymbol{\sigma}_{B}\left(t>t_{r}\right)\right\rangle=\hat{x} \cos \left(\Omega t-\frac{\Omega}{2} t_{r}\right) \operatorname{sen} \frac{\Omega}{2} t_{r}-\hat{y} \cos \frac{\Omega}{2} t_{r}+\hat{z} \operatorname{sen}\left(\Omega t-\frac{\Omega}{2} t_{r}\right) \operatorname{sen} \frac{\Omega}{2} t_{r}
$$

Se considerarmos que o buraco pode se recombinar com igual probabilidade a qualquer um dos elétrons, seja aquele de spin para cima ou aquele de spin para baixo, segue, por exemplo, que a componente $z$ do momento magnético é dada pela seguinte ponderação:

$$
\left\langle\sigma_{z}\left(t>t_{r}\right)\right\rangle=\frac{\left\langle\sigma_{A z}\left(t>t_{r}\right)\right\rangle+\left\langle\sigma_{B z}\left(t>t_{r}\right)\right\rangle}{2}=-\frac{1}{2} \cos \Omega t
$$

a qual é independente de $t_{r}$, e idêntica ao resultado obtido em (4.23). Isso quer dizer que, após a recombinação, o movimento observado é apenas uma continuação da dinâmica de spin da componente não-excitada da função de onda eletrônica ${ }^{8}$.

Tal resultado pode ser interpretado fisicamente se considerarmos que para $t>t_{r}$, $\left\langle\sigma_{z}(t)\right\rangle$ continua a oscilar com a mesma fase da componente de spin não-excitada, uma vez que a contribuição do spin adicional - gerado pela interação com o pulso laser torna-se aleatória após a recombinação do tríon, acarretando numa contribuição nula para a média.

Tal suposição de aleatoriedade no processo de recombinação da parte excitada implica que apenas a componente não-excitada pelo pulso de luz contribui para magnetização, e considerando-se que o movimento de precessão se inicia de um estado de spin para baixo $(\downarrow)$, segue como previsão do modelo a existência de uma única fase constante e igual a zero $(\phi=0)$ independente da intensidade do campo aplicado, fator giromagnético do elétron (ou buraco), gap de energia das ilhas quânticas e tempo de vida do tríon. No entanto, tal previsão contradiz os resultados obtidos experimentalmente, conforme verificado por Henriques et al[35].

\footnotetext{
${ }^{8}$ Basta comparar (4.31) com a componente $z$ de (4.23)
} 
Tendo isso em vista, um modelo mais robusto e coeso é proposto na seção seguinte de forma a contornar a discrepância existente entre o modelo teórico e as observações experimentais.

\subsection{Efeito de Precessão do Buraco na Polarização do Spin Eletrônico}

Da seção 4.3 anterior, assumimos que o buraco pode vir a capturar com igual probabilidade qualquer um dos dois elétrons participantes do tríon. No entanto, se considerarmos que o buraco precessiona coerentemente, segue que no instante de recombinação, $t=t_{r}$, o spin do buraco apresentará uma orientação específica, e por consequência as probabilidades de captura deixam de ser as mesmas e tornam-se dependentes da dinâmica de precessão do buraco.

De maneira a incorporar tal observação ao modelo, partamos da hamiltoniana para o buraco no trion [39]:

$$
\mathcal{H}_{h}=\frac{\mu_{B}}{2} \boldsymbol{\sigma} \cdot \boldsymbol{g}_{\boldsymbol{h}} \cdot \boldsymbol{B}
$$

na qual $\boldsymbol{\sigma}=\sigma_{x} \hat{\boldsymbol{x}}+\sigma_{y} \hat{\boldsymbol{y}}+\sigma_{z} \hat{\boldsymbol{z}}$ e $\sigma_{i}$ são as matrizes de Pauli para um buraco com pseudo- $\operatorname{spin} 1 / 2$.

Se tomarmos o eixo $x$ ao longo da direção [110], e o eixo $y$ ao longo de [1̄o], segue que, nesta base, o tensor $\boldsymbol{g}_{\boldsymbol{h}}$ adquire uma forma diagonalizada, ou seja, $\boldsymbol{g}_{\boldsymbol{h}}=$ $\operatorname{diag}\left(g_{h x}, g_{h y}, g_{h z}\right)$. Em particular, se considerarmos um campo magnético $\boldsymbol{B}$ aplicado sobre o plano $x y$, com um ângulo $\theta$ em relação à direção $x$, temos que a hamiltoniana de spin do buraco pode ser reescrita na forma:

$$
\begin{gathered}
\mathcal{H}_{h}=\frac{\mu_{B}}{2} \boldsymbol{\sigma} \cdot\left(\begin{array}{ccc}
g_{h x} & 0 & 0 \\
0 & g_{h y} & 0 \\
0 & 0 & g_{h z}
\end{array}\right) \cdot\left(\begin{array}{c}
B \cos \theta \\
B \operatorname{sen} \theta \\
0
\end{array}\right) \\
\mathcal{H}_{h}=\frac{\mu_{B} B}{2} g_{h x} \cos \theta \sigma_{x}+\frac{\mu_{B} B}{2} g_{h y} \operatorname{sen} \theta \sigma_{y}
\end{gathered}
$$




$$
\mathcal{H}_{h}=\frac{\hbar}{2} \Omega_{h x} \cos \theta \sigma_{x}+\frac{\hbar}{2} \Omega_{h y} \operatorname{sen} \theta \sigma_{y}
$$

onde $\Omega_{h x, y}=g_{h x, y} \frac{e B}{2 m_{0}}$ são as frequências de precessão do buraco para cada uma das direções envolvidas.

Por conseguinte, o operador de evolução temporal do spin do buraco torna-se

$$
\exp \left(-\frac{i t}{\hbar} \mathcal{H}_{h}\right)=\exp \left(-\frac{i t}{2} \Omega_{h x} \cos \theta \sigma_{x}-\frac{i t}{2} \Omega_{h y} \operatorname{sen} \theta \sigma_{y}\right)
$$

e usando-se do mesmo procedimento efetuado em (4.12) bem como fazendo-se uso das propriedade de potenciação (4.13), concluímos que

$$
e^{-\frac{i t}{\hbar} \mathcal{H}_{h}}=\cos A_{x} \cos A_{y} \mathbb{I}-i \operatorname{sen} A_{x} \cos A_{y} \sigma_{x}-i \cos A_{x} \operatorname{sen} A_{y} \sigma_{y}-i \operatorname{sen} A_{x} \operatorname{sen} A_{y} \sigma_{z}
$$

onde $A_{x}=A_{x}(t)=\frac{1}{2} \Omega_{h x} t \cos \theta$ e $A_{y}=A_{y}(t)=\frac{1}{2} \Omega_{h y} t \operatorname{sen} \theta$. Desta maneira, ao aplicarmos esse operador de evolução sobre a função de spin do buraco em $\mathrm{t}=0$ (4.18), obtemos que

$\chi_{h}(t>0)=\cos A_{x} \cos A_{y}|\Uparrow\rangle-i \operatorname{sen} A_{x} \cos A_{y}|\Downarrow\rangle-i \cdot(i) \cos A_{x} \operatorname{sen} A_{y}|\Downarrow\rangle-i \operatorname{sen} A_{x} \operatorname{sen} A_{y}|\Uparrow\rangle \quad{ }^{9} ;$

Sendo assim, no instante de recombinação do exciton, $t=t_{r}$, a função de precessão de spin do buraco assume a forma

$$
\chi_{h}\left(t=t_{r}\right)=a_{h}|\Uparrow\rangle+b_{h}|\Downarrow\rangle
$$

onde os coeficientes são dados por $a_{h}=a_{h}\left(t_{r}\right)=\cos A_{x} \cos A_{y}-i \operatorname{sen} A_{x} \operatorname{sen} A_{y} \mathrm{e}$ $b_{h}=b_{h}\left(t_{r}\right)=\cos A_{x} \operatorname{sen} A_{y}-i \operatorname{sen} A_{x} \cos A_{y} \cdot{ }^{10}$

Da relação (4.37) anterior, concluímos que a probabilidade de o buraco ser encontrado com spin para cima em $t_{r}$ (ou seja, a probabilidade de captura do elétron com spin para baixo) é dada por $\left|a_{h}\right|^{2}=\left(\cos A_{x} \cos A_{y}\right)^{2}+\left(\operatorname{sen} A_{x} \operatorname{sen} A_{y}\right)^{2}$, enquanto que a

\footnotetext{
${ }^{9}$ Uma vez que $\sigma_{x}|\Uparrow\rangle=|\Downarrow\rangle, \sigma_{y}|\Uparrow\rangle=i|\Downarrow\rangle$ e $\sigma_{z}|\Uparrow\rangle=|\Uparrow\rangle$

${ }^{10}$ Aqui $A_{x}$ e $A_{y}$ estão avaliados no instante de recombinação do trion $t_{r}$, ou seja, $A_{x}=A_{x}\left(t_{r}\right)$ e $A_{y}=A_{y}\left(t_{r}\right)$
} 
probabilidade de o buraco apontar para baixo em $t_{r}$ (isto é, a probabilidade de captura do elétron com spin para cima) é dada por $\left|b_{h}\right|^{2}=\left(\cos A_{x} \operatorname{sen} A_{y}\right)^{2}+\left(\operatorname{sen} A_{x} \cos A_{y}\right)^{2}$.

Portanto, a magnetização média do QD ao longo do eixo de polarização do pulso laser (eixo $z$ ) após a recombinação do tríon é dada por:

$$
\left\langle\sigma_{z}\left(t>t_{r}\right)\right\rangle=\left|b_{h}\right|^{2}\left\langle\sigma_{A z}\right\rangle+\left|a_{h}\right|^{2}\left\langle\sigma_{B z}\right\rangle \quad 11
$$

onde $\left\langle\sigma_{A z}\right\rangle$ e $\left\langle\sigma_{B z}\right\rangle$ são os mesmos usados no cálculo de (4.31).

Por conveniência, podemos reescrever $\left|a_{h}\right|^{2}$ e $\left|b_{h}\right|^{2}$ de forma conveniente através dos seguintes procedimentos

$$
\begin{aligned}
\left|a_{h}\right|^{2} & =\left(\cos A_{x} \cos A_{y}\right)^{2}+\left(\operatorname{sen} A_{x} \operatorname{sen} A_{y}\right)^{2} \\
& =\left[\frac{\cos \left(A_{x}+A_{y}\right)+\cos \left(A_{x}-A_{y}\right)}{2}\right]^{2}+\left[\frac{\cos \left(A_{x}-A_{y}\right)-\cos \left(A_{x}+A_{y}\right)}{2}\right]^{2} \\
& =\frac{1}{2}\left[\cos ^{2}\left(A_{x}+A_{y}\right)+\cos ^{2}\left(A_{x}-A_{y}\right)\right] \\
& =\frac{1}{2}\left[\frac{1+\cos \left[2\left(A_{x}+A_{y}\right)\right]}{2}+\frac{1+\cos \left[2\left(A_{x}-A_{y}\right)\right]}{2}\right] \\
& =\frac{1}{2}\left[1+\frac{\cos \left[2\left(A_{x}+A_{y}\right)\right]+\cos \left[2\left(A_{x}-A_{y}\right)\right]}{2}\right]
\end{aligned}
$$

Analogamente, para $\left|b_{h}\right|^{2}$ temos

$$
\left|b_{h}\right|^{2}=\frac{1}{2}\left[1-\frac{\cos \left[2\left(A_{x}+A_{y}\right)\right]+\cos \left[2\left(A_{x}-A_{y}\right)\right]}{2}\right]
$$

Por consequência, segue que

$$
\left\langle\sigma_{z}\left(t>t_{r}\right)\right\rangle=\frac{\left\langle\sigma_{A z}\right\rangle+\left\langle\sigma_{B z}\right\rangle}{2}+\frac{\cos \left[2\left(A_{x}+A_{y}\right)\right]+\cos \left[2\left(A_{x}-A_{y}\right)\right]}{4}\left[\left\langle\sigma_{B z}\right\rangle-\left\langle\sigma_{A z}\right\rangle\right]
$$

Dos resultados obtidos em (4.29), (4.30) e (4.31) concluímos que

$$
\left\langle\sigma_{z}\left(t>t_{r}\right)\right\rangle=-\frac{1}{2} \cos \Omega t+\frac{\cos \left[2\left(A_{x}+A_{y}\right)\right]+\cos \left[2\left(A_{x}-A_{y}\right)\right]}{4} \cos \Omega\left(t-t_{r}\right)
$$

\footnotetext{
${ }^{11}$ Note que os coeficientes $\left|a_{h}\right|^{2}$ e $\left|b_{h}\right|^{2}$ atuam como fatores de "peso"no cálculo para a média. Na seção 4.4, ambos os termos citados eram equivalentes, tendo em vista a igual probabilidade de captura dos elétrons participantes do tríon.
} 
Definindo-se $\Omega_{1}=2 \frac{A+B}{t_{r}}=\Omega_{h x} \cos \theta+\Omega_{h y} \operatorname{sen} \theta$ e $\Omega_{2}=2 \frac{A-B}{t_{r}}=\Omega_{h x} \cos \theta-\Omega_{h y} \operatorname{sen} \theta$ chegamos a

$$
\begin{array}{r}
\left\langle\sigma_{z}\left(t>t_{r}\right)\right\rangle=-\frac{1}{2} \cos \Omega t+\frac{\cos \Omega_{1} t_{r}+\cos \Omega_{2} t_{r}}{4} \cos \Omega\left(t-t_{r}\right) \\
=-\frac{1}{2} \cos \Omega t+\sum_{i=1}^{2} \frac{\cos \left[\Omega t-\left(\Omega-\Omega_{1}\right) t_{r}\right]+\cos \left[\Omega t-\left(\Omega+\Omega_{1}\right) t_{r}\right]}{8}
\end{array}
$$

O tempo de recombinação do par elétron-buraco apresenta um comportamento estocástico, ou seja, de origem em eventos aleatórios, e portanto, a solução final deve ser a média estatística sobre uma distribuição de $t_{r}$. Diferentemente da equação (4.31), a nova expressão para $\left\langle\sigma_{z}(t)\right\rangle$ depende do instante de recombinação $t_{r}$. Desta maneira, esse último resultado deve ser ponderado sobre a distribuição de probabilidades de $t_{r}$.

A probabilidade de que uma recombinação ocorra no intervalo $\left[t_{r}, t_{r}+d t_{r}\right]$ é descrita por

$$
d P\left(t_{r}\right)=\frac{e^{-t_{r} / \tau} d t_{r}}{\tau}
$$

Onde $\tau$ é o tempo de recombinação médio do complexo tríon, da ordem de $500 \mathrm{ps}$ segundo Varwig [36] ou 360 ps segundo Greilich [40]. A resposta média é definida, portanto, como

$$
\overline{\left\langle\sigma_{z}(t)\right\rangle}=\int_{0}^{\infty}\left\langle\sigma_{z}(t)\right\rangle d P\left(t_{r}\right)
$$

No entanto, a função $\left\langle\sigma_{z}(t)\right\rangle$ é definida diferentemente para os intervalos $t<t_{r}$ e $t>t_{r}, \log 0$

$$
\begin{array}{r}
\overline{\left\langle\sigma_{z}(t)\right\rangle}=\int_{0}^{t}\left(\sigma_{z} \text { para } t_{r}<t\right) d P\left(t_{r}\right)+\int_{t}^{\infty}\left(\sigma_{z} \text { para } t_{r}>t\right) d P\left(t_{r}\right) \\
=\int_{0}^{t} \sigma_{z}\left(t>t_{r}\right) \frac{e^{-t_{r} / \tau} d t_{r}}{\tau}+\int_{t}^{\infty} \sigma_{z}\left(t<t_{r}\right) \frac{e^{-t_{r} / \tau} d t_{r}}{\tau}
\end{array}
$$

Em particular, se quisermos $\overline{\left\langle\sigma_{z}(t)\right\rangle}$ no limite de $t \gg t_{r}$, podemos supor que a exponencial $e^{-t_{r} / \tau}$ seja efetivamente nula para $t=t_{r}$, e consequentemente podemos 
expandir o limite da primeira integral de (4.46) para infinito e omitir a contribuição referente a segunda integral da mesma equação:

$$
\overline{\left\langle\sigma_{z}(t \gg \tau)\right\rangle}=\int_{0}^{\infty}\left\langle\sigma_{z}\left(t>t_{r}\right)\right\rangle \frac{e^{-t_{r} / \tau} d t_{r}}{\tau}
$$

Substituindo (4.43) na equação anterior, segue que

$$
\begin{aligned}
\overline{\left\langle\sigma_{z}(t \gg \tau)\right\rangle} & =-\frac{1}{2} \cos \Omega t \int_{0}^{\infty} d P\left(t_{r}\right)+\sum_{i=1}^{2} \int_{0}^{\infty} \frac{\cos \left[\Omega t-\left(\Omega-\Omega_{i}\right) t_{r}\right]+\left[\cos \Omega t-\left(\Omega+\Omega_{i}\right) t_{r}\right]}{8} d P\left(t_{r}\right) \\
& =-\frac{1}{2} \cos \Omega t+\sum_{i=1}^{2} \int_{0}^{\infty} \frac{\cos \left[\Omega t-\left(\Omega-\Omega_{i}\right) t_{r}\right]+\left[\cos \Omega t-\left(\Omega+\Omega_{i}\right) t_{r}\right]}{8} d P\left(t_{r}\right)
\end{aligned}
$$

Após alguns procedimentos algébricos chegamos ao seguinte resultado final:

$$
\overline{\left\langle\sigma_{z}(t \gg \tau)\right\rangle}=-R \cos (\Omega t+\phi)
$$

no qual

$$
\left\{\begin{array}{l}
R=\frac{1}{2} \sqrt{\sigma_{1}^{2}+\sigma_{2}^{2}+2 \sigma_{1} \sigma_{2} \cos \left(\phi_{2}-\phi_{1}\right)} ; \\
\sigma_{i}=\frac{1}{4} \sqrt{\operatorname{sen}^{2} \theta_{i+}+\operatorname{sen}^{2} \theta_{i-}+2 \operatorname{sen} \theta_{i+} \operatorname{sen} \theta_{i-} \cos \left(\theta_{i+}-\theta_{i-}\right)} \\
\phi_{i}=\frac{\pi}{2}-\theta_{i+}+\operatorname{arctg} \frac{\operatorname{sen} \theta_{i-} \operatorname{sen}\left(\theta_{i+}-\theta_{i-}\right)}{\operatorname{sen} \theta_{i+}+\operatorname{sen} \theta_{i-} \cos \left(\theta_{i+}-\theta_{i-}\right)} ; \\
\theta_{i \pm}=\operatorname{arctg}\left(\Omega \pm \Omega_{i}\right) \tau ; \quad \Omega=g \frac{e B}{2 m_{0}} ; \\
\Omega_{1}=\Omega_{h x} \cos \theta+\Omega_{h y} \operatorname{sen} \theta ; \quad \Omega_{2}=\Omega_{h x} \cos \theta-\Omega_{h y} \operatorname{sen} \theta \\
\Omega_{h x}=g_{h x} \frac{e}{2 m_{0}} B ; \quad \Omega_{h y}=g_{h y} \frac{e}{2 m_{0}} B \\
\phi=\phi_{1}+\operatorname{arctg} \frac{\sigma_{2} \operatorname{sen}\left(\phi_{2}-\phi_{1}\right)}{\sigma_{1}+\sigma_{2} \cos \left(\phi_{2}-\phi_{1}\right)}
\end{array}\right.
$$

Em particular, os resultados aqui presentes referem-se a modelagem para uma única ilha quântica, a passagem para um ensemble é demonstrada no Apêndice C, no qual denota-se o aparecimento do termo de amortecimento exponencial conforme apresentado em (2.51). 
Os gráficos da amplitude e fase referentes ao termo não-amortecido das oscilações de $\overline{\left\langle\sigma_{z}(t)\right\rangle}$ - equação (4.49) - são apresentados em função da intensidade do campo magnético na figura 4.4 .
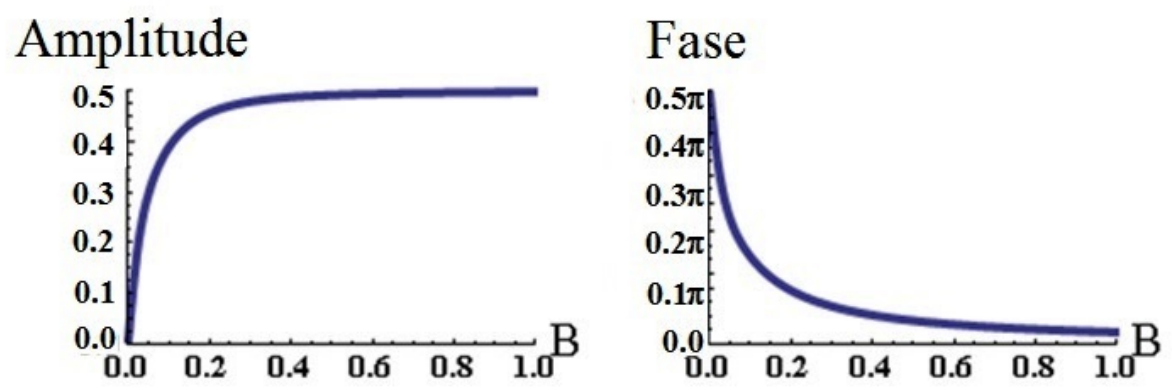

Figura 4.4: Amplitude e fase das oscilações referentes à equação (4.49). Os parâmetros empregados foram obtidos segundo a referência [30] e são dados por $g_{e}=0,53, g_{h x}=$ 0,08 e $g_{h y}=0,23$. Em particular, o eixo horizontal é dado em unidades de Tesla para o caso de um campo magnético na direção [1]̄0];

Como podemos perceber, a fase tende a 0 quando $B \rightarrow \infty$ - ou simplesmente para quando $\Omega \tau \rightarrow \infty$ - tendo em vista que, nesse caso, o buraco completa um número suficientemente grande de ciclos/voltas de precessão durante o tempo de vida $\tau$ do tríon, o que significa que no instante de recombinação, o buraco tem igual probabilidade de apontar em qualquer direção, e portanto, igual probabilidade de captura de qualquer um dos dois elétrons constituintes do tríon, retornando ao resultado descrito na seção 4.3.

Contudo, para baixa intensidade de campo, a precessão torna-se quase ausente, e portanto, nenhuma magnetização ao longo da direção $\hat{z}$ é gerada. Por consequência, a amplitude da oscilação tende à zero e a fase tende a $\phi=\pi / 2$, o que corresponde a uma direção perpendicular ao eixo $\hat{z}$. 


\subsection{Modelo do Buraco Estático}

Do exposto anteriormente, segue que uma descrição detalhada da fase da magnetização de uma dada ilha requer um tratamento quântico completo da evolução temporal de spin do buraco e dos elétrons participantes do sistema. Esses últimos, em particular, podem ser obtidos por meio da resolução da equação de Schrödinger dependente do tempo, como realizado no modelo proposto ${ }^{12}$ da seção 4.4 .

No entanto, vamos mostrar que um Modelo Simplificado - no qual apenas os elétrons precessionam, enquanto o buraco não (Modelo do Buraco Estático) - é capaz de concentrar toda a física essencial e fornecer uma excelente aproximação para com os dados experimentais e resultados do Modelo Completo.

Além disso, o Modelo Simplificado tem a enorme vantagem de acarretar em expressões concisas e de melhor interpretação física, diferentemente das relações derivadas segundo o Modelo Completo, que considera a contribuição da precessão do buraco e da anisotropia do fator- $g[30,41,42]$.

Figura 4.5: Balança esquemática voltada a comparar os modelos Simplificado e Completo. Observe que o equilíbrio pende para o lado esquerdo salien-

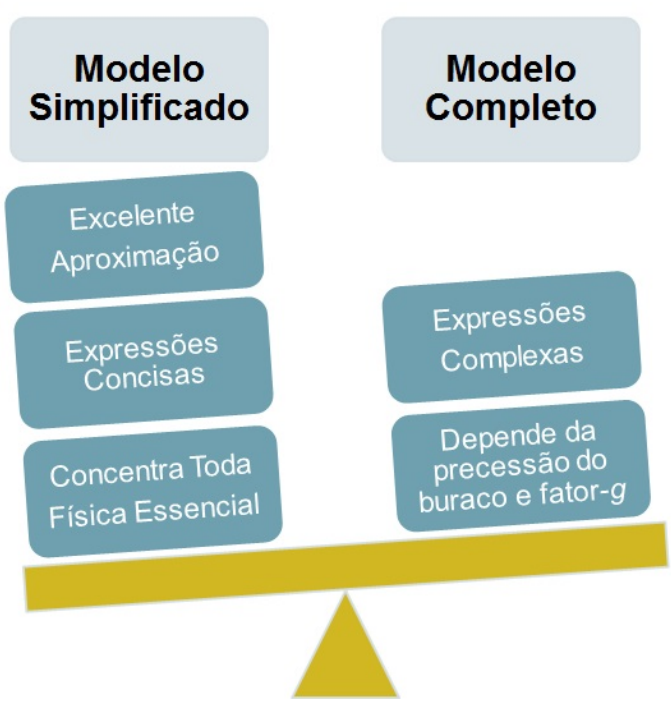
tando a vantagem desse modelo em relação ao outro.

O Modelo do Buraco Estático em si consiste em retornar à construção teórica

\footnotetext{
${ }^{12}$ Pelo fato desse modelo considerar o efeito de precessão do buraco na dinâmica de spin, o mesmo será referido como Modelo Completo na Geração de Coerência de Spin.
} 
descrita na seção 4.3 e incorporar a mesma a distribuição de tempos de recombinação da equação (4.44).

Assim sendo, vamos considerar que no instante $t=t_{r}$ o buraco estático captura o mesmo elétron fotoexcitado que o originou, isto é, o elétron com spin para baixo, conforme descrito na equação (4.25):

$$
\Psi_{B}\left(t=t_{r}\right)=\frac{1}{\sqrt{2}}\left|\uparrow-i \cos \frac{\Omega}{2} t_{r} \downarrow+i \operatorname{sen} \frac{\Omega}{2} t_{r} \uparrow\right\rangle
$$

Após a recombinação do tríon, a evolução do estado é definida segundo a equação (4.28), ou seja,

$$
\Psi_{B}\left(t>t_{r}\right)=\frac{1}{\sqrt{2}}\left\{\left[i \operatorname{sen} \frac{\Omega}{2} t+\cos \frac{\Omega}{2}\left(t-t_{r}\right)\right]|\uparrow\rangle+\left[-i \cos \frac{\Omega}{2} t+\operatorname{sen} \frac{\Omega}{2}\left(t-t_{r}\right)\right]|\downarrow\rangle\right\}
$$

O valor esperado do momento magnético para ilhas quânticas nesse estado é dado, portanto, conforme (4.30), isto é:

$$
\left\langle\boldsymbol{\sigma}_{B}\left(t>t_{r}\right)\right\rangle=\hat{x} \cos \left(\Omega t-\frac{\Omega}{2} t_{r}\right) \operatorname{sen} \frac{\Omega}{2} t_{r}-\hat{y} \cos \frac{\Omega}{2} t_{r}+\hat{z} \operatorname{sen}\left(\Omega t-\frac{\Omega}{2} t_{r}\right) \operatorname{sen} \frac{\Omega}{2} t_{r}
$$

Nesse momento, o Modelo do Buraco Estático se diferencia daquele apresentado na seção 4.3 ao incluirmos a média sobre a distribuição de tempos de recombinação, ou seja:

$$
\overline{\left\langle\sigma_{B z}(t \gg \tau)\right\rangle}=\int_{0}^{\infty}\left\langle\sigma_{B z}\left(t, t_{r}\right)\right\rangle d P\left(t_{r}\right)
$$

onde $\left\langle\sigma_{B z}\left(t, t_{r}\right)\right\rangle$ é a componente $z$ da relação (4.30) e $d P\left(t_{r}\right)$ é a distribuição descrita em (4.44).

Logo, segue que

$$
\begin{array}{r}
\overline{\left\langle\sigma_{B z}(t \gg \tau)\right\rangle}=\int_{0}^{\infty} \operatorname{sen}\left(\Omega t-\frac{\Omega t_{r}}{2}\right) \operatorname{sen} \frac{\Omega t_{r}}{2} \frac{e^{-t_{r} / \tau}}{\tau} d t_{r} \\
=\int_{0}^{\infty} \operatorname{sen} \Omega t \cos \frac{\Omega t_{r}}{2} \operatorname{sen} \frac{\Omega t_{r}}{2} \frac{e^{-t_{r} / \tau}}{\tau} d t_{r}-\int_{0}^{\infty} \cos \Omega t\left(\operatorname{sen} \frac{\Omega t_{r}}{2}\right)^{2} \frac{e^{-t_{r} / \tau}}{\tau} d t_{r} \\
=\frac{\operatorname{sen} \Omega t}{2} \int_{0}^{\infty} \operatorname{sen} \Omega t_{r} \frac{e^{-t_{r} / \tau}}{\tau} d t_{r}-\frac{\cos \Omega t}{2} \int_{0}^{\infty}\left(1-\cos \Omega t_{r}\right) \frac{e^{-t_{r} / \tau}}{\tau} d t_{r}
\end{array}
$$


Ao resolvermos as integrais e aplicarmos os limites de integração obtemos o seguinte resultado:

$$
\begin{gathered}
\overline{\left\langle\sigma_{B z}(t \gg \tau)\right\rangle}=-\frac{1}{2} \cos \Omega t+\frac{1}{2} \frac{\Omega \tau}{\left(1+\Omega^{2} \tau^{2}\right)} \operatorname{sen} \Omega t+\frac{1}{2} \frac{1}{\left(1+\Omega^{2} \tau^{2}\right)} \cos \Omega t \\
\overline{\left\langle\sigma_{B z}(t \gg \tau)\right\rangle}=\frac{1}{2} \frac{\Omega \tau}{\left(1+\Omega^{2} \tau^{2}\right)} \operatorname{sen} \Omega t-\frac{1}{2} \frac{\Omega^{2} \tau^{2}}{\left(1+\Omega^{2} \tau^{2}\right)} \cos \Omega t
\end{gathered}
$$

Tal resultado pode ser reescrito adotando-se um ângulo $\alpha$ tal qual denotado na figura 4.6 abaixo:

Figura 4.6: Geometria triangular usada na simplificação do resultado (4.52)

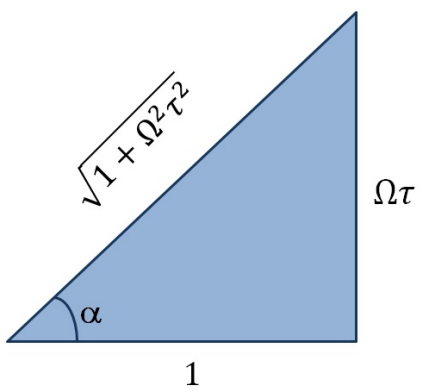

Através desse procedimento, obtemos a seguinte simplificação para a equação (4.52):

$$
\begin{aligned}
& \overline{\left\langle\sigma_{B z}(t \gg \tau)\right\rangle}=\frac{1}{2} \operatorname{sen} \alpha \cos \alpha \operatorname{sen} \Omega t-\frac{1}{2} \operatorname{sen}^{2} \alpha \cos \Omega t \\
= & \frac{1}{2} \operatorname{sen} \alpha \operatorname{sen}(\Omega t-\alpha)=-\frac{1}{2} \operatorname{sen} \alpha \cos (\Omega t-\alpha+\pi / 2) \\
\therefore & \overline{\left\langle\sigma_{B z}(t \gg \tau)\right\rangle}=-\frac{1}{2} \frac{\Omega \tau}{\sqrt{1+\Omega^{2} \tau^{2}}} \cos (\Omega t+\phi)
\end{aligned}
$$

Onde a fase da oscilação é dada por $\phi=\pi / 2-\alpha$, ou seja,

$$
\phi=\frac{\pi}{2}-\operatorname{arctg} \Omega \tau
$$

Vale salientar que o termo oscilante na equação (4.53) descreve o movimento de precessão do momento magnético eletrônico associado à superposição das partes fotoexcitada e não-excitada da função de onda de $\operatorname{spin}^{13}$.

\footnotetext{
${ }^{13} \mathrm{~A}$ expansão do resultado (4.53) de uma ilha isolada para um ensemble de QD é descrito no Apêndice C, no qual denota-se o aparecimento do termo de amortecimento exponencial conforme apresentado em (2.51).
} 


\subsection{Resultados Experimentais e Comparação com Mo- delos Teóricos}

O gráfico da fase da magnetização em função do campo magnético aplicado é apresentado na figura 4.7, a seguir, juntamente com os dados obtidos experimentalmente:

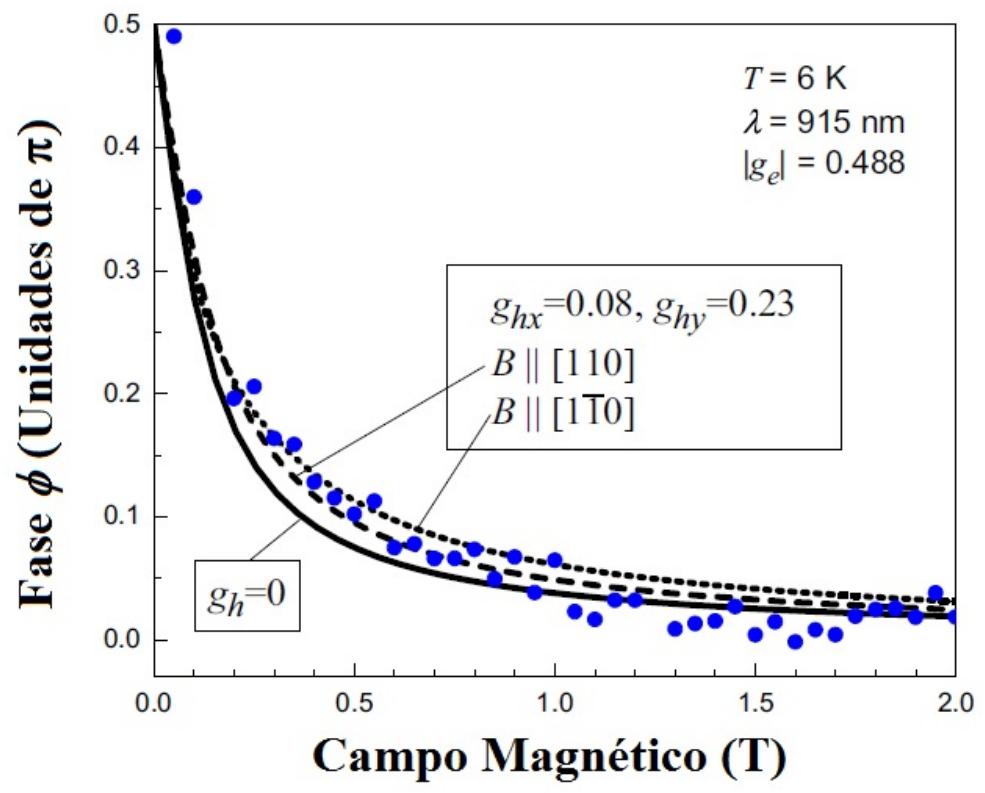

Figura 4.7: Fase da magnetização em função da intensidade do campo magnético B. Os pontos azuis representam os dados obtidos experimentalmente para uma amostra com $\left|g_{e}\right|=0$, 488; A linha contínua representa a curva teórica de $g_{h}=0$ (Buraco Estático) para $\tau=0,2 \mathrm{~ns}$; As linhas tracejada e pontilhada representam, respectivamente, as curvas teóricas do Modelo Completo para um campo magnético aplicado na direção [110] e [110], considerando-se $g_{h x}=0,08, g_{h y}=0,23$ e $\tau=0,2$ ns.

Como podemos perceber, a equação (4.54) reproduz consideravelmente bem o comportamento do conjunto de pontos experimentais tanto no limite de campos altos, isto é, $\phi \rightarrow 0$ quando $B \rightarrow \infty$, quanto no limite de campos baixos, ou seja, $\phi \rightarrow \frac{\pi}{2}$ quando $B \rightarrow 0$.

Além disso, podemos notar o efeito da precessão do buraco na fase da oscilação da magnetização, principalmente na região intermediária de campos magnéticos, salientando o efeito anisotrópico do buraco na descrição dos pontos experimentais.

Os ajustes da fase em função do campo magnético são realizados usando um único parâmetro variável, no caso, o tempo de recombinação do tríon $(\tau)$. Para pequenos 
valores de campo, tais como utilizados na figura 4.7, as funções de onda do buraco e dos elétrons confinados às ilhas quânticas fotoexcitadas são essencialmente inalteradas de seus valores em $B=0$, uma vez que as forças de Lorentz que atuam sobre as partículas confinadas são muito menores do que as forças Coulombianas associadas ao potencial do QD. Portanto, o valor de $\tau=0,2$ ns na figura 4.7 pode ser seguramente tomado como uma precisa estimativa do tempo de recombinação do exciton, viabilizando o emprego dos modelos propostos como novas técnicas na determinação dos tempos de recombinação.

O tempo de vida estimado por esses métodos corresponde a um tríon que se recombina com a mesma energia da luz de excitação, ou seja, um tríon ressonante. Isso contrasta com o típico tempo de recombinação obtido a partir da fotoluminescência resolvida no tempo [40], em que a excitação ressonante é dificultada pelo espalhamento de luz, e se emprega, portanto, uma excitação não-ressonante, em que o tempo de vida do tríon é fortemente influenciado pela relaxação e possível formação de dark excitons ${ }^{14}$. Particularmente, de acordo com Kurtze et al. [44], medidas de transmissão diferencial estimam que o tempo de vida de um tríon não-ressonante seja duas vezes maior que um ressonante, o que então levaria o valor de $\tau=0,2$ ns obtido nesse trabalho para cerca de $\tau \approx 0,4 \mathrm{~ns}$, em concordância com os valores típicos para ilhas de (In, Ga)As/GaAs [45]. Tal passagem valida novamente a importância dos modelos teóricos desenvolvidos como técnicas para determinação do tempo de recombinação do trion.

\footnotetext{
${ }^{14} \mathrm{Um}$ exciton no qual os spins do elétron e heavy-hole (buraco com componente de $\operatorname{spin} J_{z}= \pm 3 / 2$ ) constituintes são orientados paralelamente um em relação outro é denominado "dark" exciton. Tal par de elétron-buraco não pode ser excitado (recombinado) por absorção (emissão) de um fóton, e portanto, é dito opticamente inativo. Tal como um "bright"exciton (par de elétron-buraco de spins anti-paralelos, opticamente ativo), um dark exciton é neutro, e portanto, protegido de variações elestrostáticas do ambiente que o cerca. Como resultado da forma de interação de exchange entre o elétron e o buraco, um dark exciton apresenta energia inferior a um bright exciton, o que o protege de um spin-flip (isto é, uma repentina mudança na orientação de spin) de um de seus constituintes. Por essas razões, o tempo de vida dos dark excitons é muito longo e prolonga o tempo de recombinação do tríon medido via transmissão diferencial.[43]
} 


\subsection{Interpretação Física}

O comportamento da fase $\phi$ para o Modelo do Buraco Estático pode ser interpretado por meio do seguinte diagrama de spin apresentado na figura 4.8. A descrição detalhada dessa imagem esquemática é abordada nos próximos parágrafos.

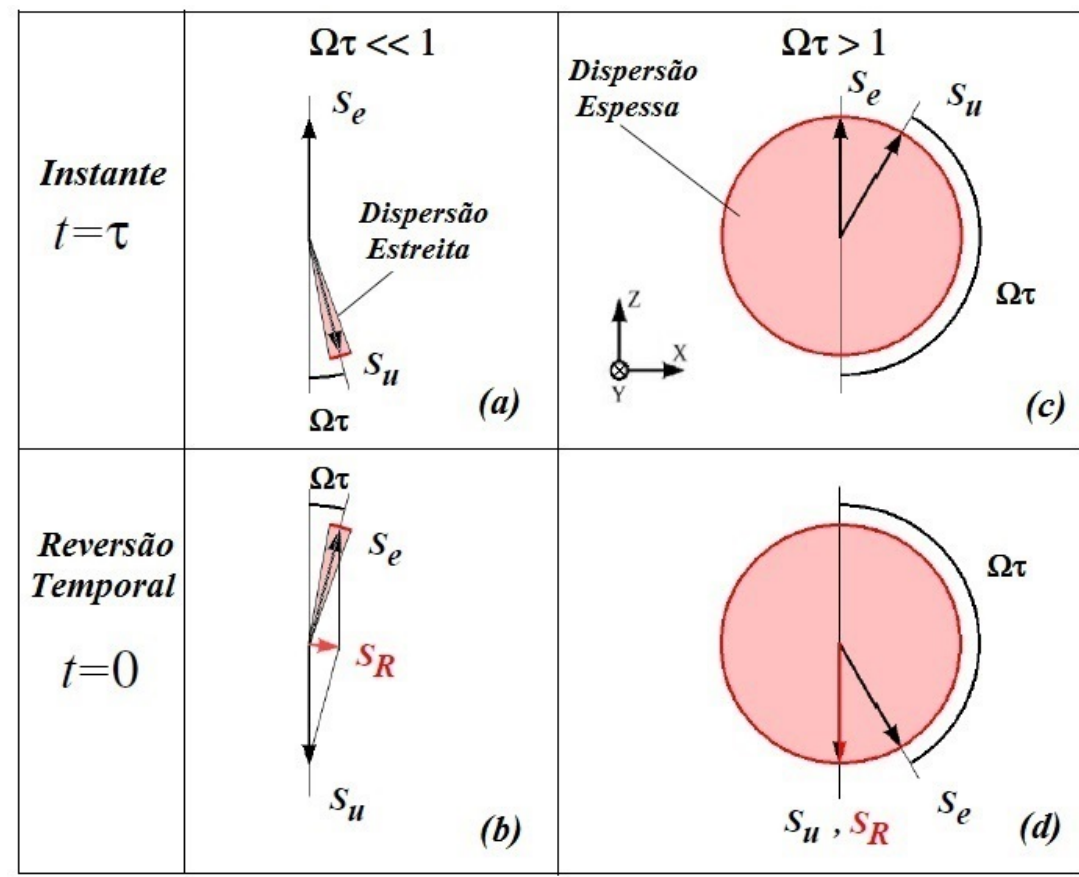

Figura 4.8: Diagrama esquemático do valor esperado dos vetores de spin no instante de recombinação médio do tríon $(t=\tau)$ - painéis superiores - e no instante $t=0$ (Reversão Temporal) - painéis inferiores. Os quadros (a) e (b) representam os vetores no limite de campo baixo, enquanto os quadros (c) e (d) representam os mesmos no limite de campo alto.

Para baixas intensidades de campo, quando $\Omega \tau \ll 1$, o movimento de precessão é suficientemente lento, portanto, no instante de recombinação do tríon, a componente de spin não-excitada da função de onda dá origem a um vetor $\boldsymbol{S}_{\boldsymbol{u}}$ rotacionado de um pequeno ângulo com relação à sua direção inicial, no caso, para baixo. Além disso, vale ressaltar que a dispersão ${ }^{15}$ no ângulo de rotação de $\boldsymbol{S}_{\boldsymbol{u}}$ entre as ilhas é pequena, tendo em vista a lenta precessão do sistema (Vide painel (a) da figura 4.8).

A fase da oscilação da magnetização é determinada pela orientação média do vetor

\footnotetext{
${ }^{15}$ Devido à distribuição de tempos de recombinação.
} 
de spin das ilhas quânticas no instante $t=0$. Tal orientação pode ser obtida por meio de uma reversão temporal dos vetores associados às componentes de spin excitada $\left(\boldsymbol{S}_{\boldsymbol{e}}\right)$ e não-excitada $\left(\boldsymbol{S}_{\boldsymbol{u}}\right)$ da função de onda, e calculando-se o vetor resultante em $t=0$.

No caso em particular de $\Omega \tau \ll 1$, o vetor $\boldsymbol{S}_{\boldsymbol{u}}$ revertido para $t=0$, de todas as ilhas, aponta para baixo, enquanto que o vetor $\boldsymbol{S}_{\boldsymbol{e}}$ aponta na direção para cima rotacionado de um pequeno ângulo com a vertical e distribuído sobre uma pequena faixa de valores ao redor. Como podemos observar no painel (b) da figura 4.8, o spin resultante $\boldsymbol{S}_{\boldsymbol{R}}=\boldsymbol{S}_{\boldsymbol{u}}+\boldsymbol{S}_{\boldsymbol{e}}$ apresenta módulo bastante reduzido e aproximadamente perpendicular a $z$, significando uma baixa amplitude de oscilação associada a uma fase $\phi=\frac{\pi}{2}$.

O mesmo procedimento pode ser aplicado para análise da fase de oscilação para altas intensidades de campo, isto é, para quando $\Omega \tau>1$. Neste caso, a precessão é suficientemente rápida, de forma tal que, na recombinação, $\boldsymbol{S}_{\boldsymbol{u}}$ esteja rotacionado de um ângulo grande no que diz respeito à sua direção inicial, além de apresentar uma larga dispersão angular (Vide painel (c) da figura 4.8). Ao realizarmos uma reversão temporal para $t=0$, segue que o vetor $\boldsymbol{S}_{\boldsymbol{u}}$, de todas as ilhas, aponta para baixo, sendo sua dispersão angular transferida para $\boldsymbol{S}_{\boldsymbol{e}}$, o qual, portanto, não contribui na $\underline{\text { média }}^{16}$ para o vetor resultante $\boldsymbol{S}_{\boldsymbol{R}}$ (Painel (d) da figura 4.8). Logo, para altos valores de campo magnético, o spin resultante do processo de reversão temporal aponta na mesma direção de $\boldsymbol{S}_{\boldsymbol{u}}$, que corresponde a uma oscilação de fase $\phi=0$ e de máxima amplitude.

Portanto, a descrição do Modelo do Buraco Estático implica em uma magnetização de amplitude constante e fase $\phi=0$ no limite de $B \rightarrow \infty$, e em uma amplitude

\footnotetext{
${ }^{16}$ Uma vez que as componentes associadas ao vetor $\boldsymbol{S}_{\boldsymbol{e}}$ anulam-se aos pares tendo em vista a grande dispersão de valores angulares que tal vetor pode apresentar.
} 
tendendo a zero e fase $\phi=\frac{\pi}{2}$ na situação de $B=0$.

Se no entanto, considerarmos o movimento de precessão do buraco (Modelo Completo), segue que o ângulo revertido no tempo associado à parte excitada da função de spin é, em média, modificado pelo fator $\Omega_{h} \tau$, onde $\Omega_{h}=g_{h} \mu_{B} B / \hbar$ é a frequência de precessão e $g_{h}$ o fator giromagnético do buraco. Logo, nos casos limites de intensidade de campo magnético, o efeito da inserção da precessão do buraco não modifica os comportamentos de fase e amplitude previstos segundo o Modelo Estático. Entretanto, para valores intermediários de campo, o movimento do buraco causa um pequeno efeito na descrição da fase de oscilação, conforme mostrado na figura 4.7. 


\section{Capítulo 5}

\section{Conclusões}

Em suma, desenvolvemos uma modelagem quântica da magnetização induzida pela luz em um conjunto de ilhas quânticas (QD) carregadas, que leva em consideração a coerência de spin dos buracos fotoexcitados.

O primeiro modelo proposto, dito Modelo Completo, teve em vista o efeito de precessão do buraco na polarização média do spin eletrônico, o que requereu um total tratamento quântico da evolução temporal de spin do buraco e dos elétrons participantes do sistema. Tal modelo resultou numa ótima descrição da fase de magnetização com expressões dependentes da anisotropia de fator- $g$. No entanto, apesar da excelente concordância com os dados experimentais, os resultados obtidos por essa via apresentaram uma complexa interpretação física devido aos diversos parâmetros envolvidos nessa abordagem.

Dessa maneira, uma modelagem alternativa foi proposta, na qual apenas os elétrons precessionavam, enquanto os buracos mantinham-se estáticos (Modelo simplificado). Nesse desenvolvimento, uma expressão concisa para a fase da oscilação da magnetização na presença de campo magnético foi obtida. Particularmente, nessa abordagem, a fase apresentou uma evolução gradual de $\phi=\frac{\pi}{2}$ na ausência de campo $(B=0)$ para $\phi=0$ no limite de altas intensidades $(B \rightarrow \infty)$. Demonstramos, ainda, a validação da teoria desenvolvida ao confrontamos e compararmos os resultados previstos pelo 
modelo com aqueles provenientes de dados experimentais. Além disso, os fundamentos teóricos descritos podem ser expandidos para ilhas quânticas com buracos armadilhados (ilhas tipo- $p$ ), o que torna o modelo uma peça chave no entendimento generalizado do processo de coerência de spin em ilhas quânticas carregadas.

Finalmente, sugerimos que o método empregado em nossa teoria fornece uma nova técnica na medição do tempo de vida médio (ou tempo de recombinação médio) do tríon em um conjunto de ilhas quânticas, que combinado às medidas de fase em função do campo magnético, pode ser utilizado na medição da dependência do tempo de recombinação para com a energia de emissão das ilhas quânticas. 


\section{Capítulo 6}

\section{Perspectivas Futuras}

As perspectivas futuras quanto ao prosseguimento desse trabalho incluem os seguintes tópicos:

- Expansão dos resultados obtidos considerando-se excitações do estado fundamental $\left(e_{s}\right)$ para o estado tripleto $\left(T_{p}\right)$;

- Realização de experimentos envolvendo amostras com buracos armadilhados em ilhas quânticas (ilhas tipo- $p$ );

- Obtenção experimental da dependência do tempo de recombinação $(\tau)$ para com a energia do gap $\left(E_{g}\right)$ das ilhas quânticas; 


\section{Apêndice A}

\section{Hamiltoniana Dipolar}

Partindo-se da hamiltoniana de interação dipolar dada por:

$$
\mathcal{H}=\frac{\mu_{0}}{4 \pi}\left\{\frac{\boldsymbol{\mu}_{\boldsymbol{e}} \cdot \boldsymbol{\mu}_{\boldsymbol{n}}}{r^{3}}-\frac{3\left(\boldsymbol{\mu}_{\boldsymbol{e}} \cdot \boldsymbol{r}\right)\left(\boldsymbol{\mu}_{\boldsymbol{n}} \cdot \boldsymbol{r}\right)}{r^{5}}\right\}
$$

Onde $\boldsymbol{\mu}_{\boldsymbol{e}}=-g_{e} \mu_{B} \boldsymbol{I}_{\boldsymbol{e}} / \hbar$ é o momento magnético do elétron; $\boldsymbol{\mu}_{\boldsymbol{n}}=g_{n} \mu_{n} \boldsymbol{I}_{\boldsymbol{n}} / \hbar$ é o momento magnético do núcleo; $\mu_{B}$ é o magneton de Bohr; $\mu_{n}$ é o magneton nuclear; $\boldsymbol{r}$ corresponde ao vetor que conecta ambos os momentos de dipolo mencionados; $\boldsymbol{I}_{\boldsymbol{e}}$ representa o operador de spin do elétron; $\boldsymbol{I}_{\boldsymbol{n}}$ corresponde ao operador de spin nuclear; $g_{e}$ e $g_{n}$ são, nessa ordem, os fatores giromagnéticos eletrônico e nuclear.

Escrevendo-se $\boldsymbol{\mu}_{\boldsymbol{e}}$ e $\boldsymbol{\mu}_{\boldsymbol{n}}$ em componentes cartesianas, segue que a hamiltoniana enunciada apresenta a forma:

$\mathcal{H}=-\frac{\mu_{0}}{4 \pi} \frac{\mu_{e} \mu_{n} g_{e} g_{n}}{\hbar^{2} r^{3}}\left\{\left(I_{e x} I_{n x}+I_{e y} I_{n y}+I_{e z} I_{n z}\right)-3 \frac{\left(I_{e x} x+I_{e y} y+I_{e z} z\right)}{r^{2}}\left(I_{n x} x+I_{n y} y+I_{n z} z\right)\right\}$

Em particular, podemos expressar $I_{e x}$ e $I_{e y}$ em termos dos operadores de abaixamento e levantamento - respectivamente, $I_{e}^{-}$e $I_{e}^{+}$- através da seguinte relação:

$$
I_{e}^{ \pm}=I_{e x} \pm i I_{e y}
$$

De maneira completamente análoga, podemos utilizar o mesmo procedimento para as componentes de spin de origem nuclear, e escrever:

$$
I_{n}^{ \pm}=I_{n x} \pm i I_{n y}
$$


Destas relações decorrem as seguintes expressões:

$$
\left\{\begin{array} { l } 
{ I _ { e x } = \frac { I _ { e } ^ { + } + I _ { e } ^ { - } } { 2 } } \\
{ I _ { e y } = \frac { I _ { e } ^ { + } - I _ { e } ^ { - } } { 2 i } }
\end{array} \quad \left\{\begin{array}{l}
I_{n x}=\frac{I_{n}^{+}+I_{n}^{-}}{2} \\
I_{n y}=\frac{I_{n}^{+}-I_{n}^{-}}{2 i}
\end{array}\right.\right.
$$

Ao substituirmos (A.5) em (A.2), e escrevermos as coordenadas cartesianas $x, y$ e $z$ em termos das coordenadas esféricas

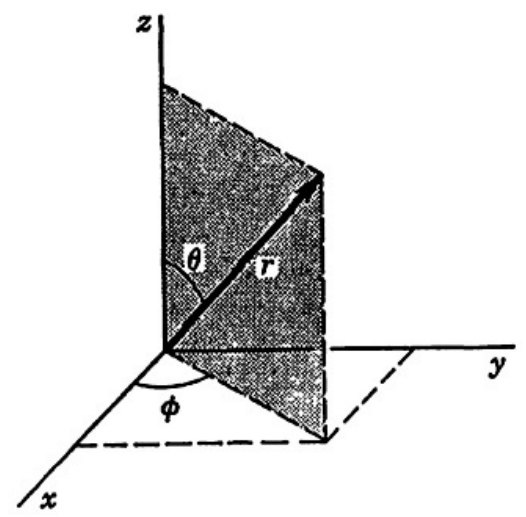

$$
\left\{\begin{array}{l}
x=r \operatorname{sen} \theta \cos \phi \\
y=r \operatorname{sen} \theta \operatorname{sen} \phi \\
z=r \cos \theta
\end{array}\right.
$$

Figura A.1: Relação entre as coordenadas retangulares $x, y$ e $z$ - que descrevem a posição eletrônica em relação ao núcleo - e as coordenadas esféricas $r, \theta$ e $\phi$. [16]

Podemos desenvolver uma hamiltoniana particularmente conveniente através das seguintes passagens

$$
\begin{aligned}
& \mathcal{H}=-\frac{\mu_{0}}{4 \pi} \frac{\mu_{e} \mu_{n} g_{e} g_{n}}{\hbar^{2} r^{3}}\left[I_{e x} I_{n x}\left(1-3 \frac{x^{2}}{r^{2}}\right)+I_{e y} I_{n y}\left(1-3 \frac{y^{2}}{r^{2}}\right)+I_{e z} I_{n z}\left(1-3 \frac{z^{2}}{r^{2}}\right)-\right. \\
&\left.-3 I_{e x} I_{n y} \frac{x y}{r^{2}}-3 I_{e x} I_{n z} \frac{x z}{r^{2}}-3 I_{e y} I_{n x} \frac{y x}{r^{2}}-3 I_{e y} I_{n z} \frac{y z}{r^{2}}-3 I_{e z} I_{n x} \frac{z x}{r^{2}}-3 I_{e z} I_{n y} \frac{z y}{r^{2}}\right] \\
& \mathcal{H}=- \frac{\mu_{0}}{4 \pi} \frac{\mu_{e} \mu_{n} g_{e} g_{n}}{\hbar^{2} r^{3}}\left[\frac{\left(I_{e}^{+}+I_{e}^{-}\right)\left(I_{n}^{+}+I_{n}^{-}\right)}{4}\left(1-3 \operatorname{sen}^{2} \theta \cos ^{2} \phi\right)-\frac{\left(I_{e}^{+}-I_{e}^{-}\right)\left(I_{n}^{+}-I_{n}^{-}\right)}{4}(1-\right. \\
&\left.-3 \operatorname{sen}^{2} \theta \operatorname{sen}^{2} \phi\right)+I_{e z} I_{n z}\left(1-3 \cos ^{2} \theta\right)+3 i \frac{\left(I_{e}^{+}+I_{e}^{-}\right)\left(I_{n}^{+}-I_{n}^{-}\right)}{4} \operatorname{sen}^{2} \theta \operatorname{sen} \phi \cos \phi- \\
&-3 \frac{\left(I_{e}^{+}+I_{e}^{-}\right)}{2} I_{n z} \operatorname{sen} \theta \cos \theta \cos \phi+3 i \frac{\left(I_{e}^{+}-I_{e}^{-}\right)\left(I_{n}^{+}+I_{n}^{-}\right)}{4} \operatorname{sen}^{2} \theta \operatorname{sen} \phi \cos \phi+ \\
&+3 i \frac{\left(I_{e}^{+}-I_{e}^{-}\right)}{2} I_{n z} \operatorname{sen} \theta \cos \theta \operatorname{sen} \phi-3 I_{e z} \frac{\left(I_{n}^{+}+I_{n}^{-}\right)}{2} \operatorname{sen} \theta \cos \theta \cos \phi+ \\
&\left.+3 i I_{e z} \frac{\left(I_{n}^{+}-I_{n}^{-}\right)}{2} \operatorname{sen} \theta \cos \theta \operatorname{sen} \phi\right]
\end{aligned}
$$




$$
\begin{array}{r}
\mathcal{H}=-\frac{\mu_{0}}{4 \pi} \frac{\mu_{e} \mu_{n} g_{e} g_{n}}{\hbar^{2} r^{3}}\left[I_{e z} I_{n z}\left(1-3 \cos ^{2} \theta\right)+\frac{\left(I_{e}^{+} I_{n}^{-}+I_{e}^{-} I_{n}^{+}\right)}{4}\left(1-3 \operatorname{sen}^{2} \theta \cos ^{2} \phi+1-\right.\right. \\
\left.-3 \operatorname{sen}^{2} \theta \operatorname{sen}^{2} \phi\right)-\frac{3}{2} I_{e}^{+} I_{n z} \operatorname{sen} \theta \cos \theta(\cos \phi-i \operatorname{sen} \phi)-\frac{3}{2} I_{e z} I_{n}^{+} \operatorname{sen} \theta \cos \theta(\cos \phi-i \operatorname{sen} \phi)- \\
-\frac{3}{2} I_{e}^{-} I_{n z} \operatorname{sen} \theta \cos \theta(\cos \phi+i \operatorname{sen} \phi)-\frac{3}{2} I_{e z} I_{n}^{-} \operatorname{sen} \theta \cos \theta(\cos \phi+i \operatorname{sen} \theta)+ \\
+\frac{I_{e}^{+} I_{n}^{+}}{4}\left(-3 \operatorname{sen}^{2} \theta \cos ^{2} \phi+3 \operatorname{sen}^{2} \theta \operatorname{sen}^{2} \phi+6 i \operatorname{sen}^{2} \theta \operatorname{sen} \phi \cos \phi\right)+ \\
\left.+\frac{I_{e}^{-} I_{n}^{-}}{4}\left(-3 \operatorname{sen}^{2} \theta \cos ^{2} \phi+3 \operatorname{sen}^{2} \theta \operatorname{sen}^{2} \phi-6 i \operatorname{sen}^{2} \theta \operatorname{sen} \phi \cos \phi\right)\right]
\end{array}
$$

Ao agruparmos os termos de forma adequada, segue que

$$
\begin{array}{r}
\mathcal{H}=-\frac{\mu_{0}}{4 \pi} \frac{\mu_{e} \mu_{n} g_{e} g_{n}}{\hbar^{2} r^{3}}\left[I_{e z} I_{n z}\left(1-3 \cos ^{2} \theta\right)+\frac{\left(I_{e}^{+} I_{n}^{-}+I_{e}^{-} I_{n}^{+}\right)}{4}\left(2-3 \operatorname{sen}^{2} \theta\right)-\right. \\
-\frac{3}{2}\left(I_{e}^{+} I_{n z}+I_{e z} I_{n}^{+}\right) \operatorname{sen} \theta \cos \theta e^{-i \phi}-\frac{3}{2}\left(I_{e}^{-} I_{n z}+I_{e z} I_{n}^{-}\right) \operatorname{sen} \theta \cos \theta e^{i \phi}+ \\
\left.+\frac{I_{e}^{+} I_{n}^{+}}{4}\left(-3 \operatorname{sen}^{2} \theta \cos 2 \phi+3 i \operatorname{sen}^{2} \theta \operatorname{sen} 2 \phi\right)+\frac{I_{e}^{-} I_{n}^{-}}{4}\left(-3 \operatorname{sen}^{2} \theta \cos 2 \phi-3 i \operatorname{sen}^{2} \theta \operatorname{sen} 2 \phi\right)\right]
\end{array}
$$

Finalmente, concluímos que

$$
\begin{array}{r}
\mathcal{H}=-\frac{\mu_{0}}{4 \pi} \frac{\mu_{e} \mu_{n} g_{e} g_{n}}{\hbar^{2} r^{3}}\left[I_{e z} I_{n z}\left(1-3 \cos ^{2} \theta\right)-\frac{\left(I_{e}^{+} I_{n}^{-}+I_{e}^{-} I_{n}^{+}\right)}{4}\left(1-3 \cos ^{2} \theta\right)-\right. \\
-\frac{3}{2}\left(I_{e}^{+} I_{n z}+I_{e z} I_{n}^{+}\right) \operatorname{sen} \theta \cos \theta e^{-i \phi}-\frac{3}{2}\left(I_{e}^{-} I_{n z}+I_{e z} I_{n}^{-}\right) \operatorname{sen} \theta \cos \theta e^{i \phi}+ \\
\left.-\frac{3}{4} I_{e}^{+} I_{n}^{+} \operatorname{sen}^{2} \theta e^{2 i \phi}-\frac{3}{4} I_{e}^{-} I_{n}^{-} \operatorname{sen}^{2} \theta e^{-2 i \phi}\right]
\end{array}
$$

Ou seja, de forma simplificada, temos que a hamiltoniana dipolar apresenta a seguinte estrutura

$$
\mathcal{H}=-\frac{\mu_{0}}{4 \pi} \frac{g_{e} g_{n} \mu_{B} \mu_{n}}{\hbar^{2} r^{3}}(A+B+C+D+E+F)
$$

onde os coeficientes correspondem a:

$$
\left\{\begin{array} { l } 
{ A = I _ { e z } I _ { n z } ( 1 - 3 \operatorname { c o s } ^ { 2 } \theta ) } \\
{ B = - \frac { 1 } { 4 } ( I _ { e } ^ { + } I _ { n } ^ { - } + I _ { e } ^ { - } I _ { n } ^ { + } ) ( 1 - 3 \operatorname { c o s } ^ { 2 } \theta ) } \\
{ C = - \frac { 3 } { 2 } ( I _ { e } ^ { + } I _ { n z } + I _ { e z } I _ { n } ^ { + } ) \operatorname { s e n } \theta \operatorname { c o s } \theta e ^ { - i \phi } }
\end{array} \quad \left\{\begin{array}{l}
D=-\frac{3}{2}\left(I_{e}^{-} I_{n z}+I_{e z} I_{n}^{-}\right) \operatorname{sen} \theta \cos \theta e^{i \phi} \\
E=-\frac{3}{4} I_{e}^{+} I_{n}^{+} \operatorname{sen}^{2} \theta e^{-2 i \phi} \\
F=-\frac{3}{4} I_{e}^{-} I_{n}^{-} \operatorname{sen}^{2} \theta e^{2 i \phi}
\end{array}\right.\right.
$$

Como queríamos determinar . 


\section{Apêndice B}

\section{Identidade Algébrica}

Para provarmos a identidade vetorial enunciada em (2.21) para um vetor tridimensional $\boldsymbol{v}$ arbitrário:

$$
\int_{\text {Volume }}(\boldsymbol{\nabla} \times \boldsymbol{v}) d \tau=-\oint_{\text {Superficie }} \boldsymbol{v} \times d \boldsymbol{a}
$$

partamos, inicialmente, da substituição de $\boldsymbol{v} \rightarrow \boldsymbol{v} \times \boldsymbol{c}$ aplicado ao Teorema da Divergência, onde $\boldsymbol{c}$ corresponde a um vetor constante qualquer.

Desta maneira, segue do Teorema da Divergência que

$$
\int \boldsymbol{\nabla} \cdot(\boldsymbol{v} \times \boldsymbol{c}) d \tau=\oint(\boldsymbol{v} \times \boldsymbol{c}) \cdot d \boldsymbol{a}
$$

Empregando-se a relação vetorial

$$
\boldsymbol{\nabla} \cdot(\boldsymbol{v} \times \boldsymbol{c})=-\boldsymbol{v} \cdot(\underbrace{\boldsymbol{\nabla} \times \boldsymbol{c}}_{=0})+(\boldsymbol{\nabla} \times \boldsymbol{v}) \cdot \boldsymbol{c}=\boldsymbol{c} \cdot(\boldsymbol{\nabla} \times \boldsymbol{v})
$$

no lado esquerdo da equação (B.1), e aplicando-se a permutação cíclica $(\boldsymbol{v} \times \boldsymbol{c}) \cdot d \boldsymbol{a}=$ $\boldsymbol{c} \cdot(d \boldsymbol{a} \times \boldsymbol{v})=-\boldsymbol{c} \cdot(\boldsymbol{v} \times d \boldsymbol{a})$ no lado direito da mesma, concluímos que

$$
\int \boldsymbol{c} \cdot(\boldsymbol{\nabla} \times \boldsymbol{v})=\oint-\boldsymbol{c} \cdot(\boldsymbol{v} \times d \boldsymbol{a})
$$

Consequentemente, como $\boldsymbol{c}$ é constante, obtemos que

$$
\int(\boldsymbol{\nabla} \times \boldsymbol{v}) d \tau=-\oint \boldsymbol{v} \times d \boldsymbol{a}
$$

Como queríamos demonstrar . 


\section{Apêndice C}

\section{Sinal TRFR de um Conjunto de Ilhas Quânticas}

Os resultados obtidos no capítulo 4 referem-se a modelagem do processo de geração de coerência de spin associada a uma única ilha quântica isolada. Em nossas medições, no entanto, o que detetamos são sinais de TRFR referentes a um ensemble (ou conjunto) de ilhas quânticas que apresentam uma dispersão de tamanhos e composições químicas.

Portanto, a transição do modelo de um única ilha para um conjunto delas, se faz mediante a inclusão de uma distribuição estatística de fatores giromagnéticos $\left(g_{e}\right)$ capaz de descrever as heterogeneidades das ilhas quânticas envolvidas.

Se assumirmos uma distribuição Lorentziana ${ }^{1}$ de fatores giromagnéticos eletrônicos $g_{e}$ com valor médio $g_{e 0}$ e largura à meia altura $\Delta g_{e}$, segue que a probabilidade de uma dada ilha quântica apresentar um valor de $g_{e}$ no intervalo $\left[g_{e}, g_{e}+d g_{e}\right]$ é dada por:

$$
d P\left(g_{e}\right)=\frac{\Delta g_{e}}{\pi} \frac{1}{\left(g_{e}-g_{e 0}\right)^{2}+\left(\Delta g_{e}\right)^{2}} d g_{e}
$$

Uma vez que o fator $g_{e}$ determina a frequência de precessão $\Omega=g_{e} \frac{e B}{2 m_{0}}$, segue que a probabilidade de encontrarmos a frequência de Larmor de uma dada ilha quântica

\footnotetext{
${ }^{1} \mathrm{~A}$ interpretação da magnetização em um conjunto de ilhas quânticas é praticamente insensível à formulação matemática exata da função de dispersão, portanto, tanto uma distribuição Gaussiana quanto Lorentziana descrevem igualmente bem o conjunto de dados experimentais [30].
} 
no intervalo $[\Omega, \Omega+d \Omega]$ é:

$$
d P(\Omega)=\frac{\Delta \Omega}{\pi} \frac{1}{\left(\Omega-\Omega_{0}\right)^{2}+(\Delta \Omega)^{2}} d \Omega
$$

na qual $\Omega_{0}=\frac{e B}{2 m_{0}} g_{e 0}$ e $\Delta \Omega=\frac{e B}{2 m_{0}} \Delta g_{e}$.

Desta maneira, segue que a média eletrônica sobre o conjunto de ilhas quânticas obedece a seguinte relação:

$$
{\overline{\left\langle\sigma_{z}(t)\right\rangle_{\text {ensemble }}}} \int_{-\infty}^{+\infty} \overline{\left\langle\sigma_{z}(t)\right\rangle} d P(\Omega)
$$

onde $\overline{\left\langle\sigma_{z}(t)\right\rangle}$ refere-se aos resultados obtidos para o modelo de uma única ilha quântica, enquanto ${\overline{\left\langle\sigma_{z}(t)\right\rangle}}_{\text {ensemble }}$ refere-se ao sinal TRFR do conjunto delas.

Por simplicidade se considerarmos que todas as ilhas respondem com fase e amplitude médias definidas por $\Omega_{0}$ e descrevermos a magnetização $\overline{\left\langle\sigma_{z}(t)\right\rangle}$ como proporcional à função oscilante $\cos (\Omega t+\phi)$ (conforme os resultados obtidos no capítulo 4), basta resolvermos a seguinte integração:

$$
I=\int_{-\infty}^{+\infty} \cos (\Omega t+\phi) d P(\Omega)=\frac{\Delta \Omega}{\pi} \int_{-\infty}^{+\infty} \frac{\cos (\Omega t+\phi) d \Omega}{\left(\Omega-\Omega_{0}\right)^{2}+(\Delta \Omega)^{2}}
$$

Para resolvermos (C.4), partamos da seguinte integral no plano complexo:

$$
J=\oint_{C} \frac{e^{i(z t+\phi)} d z}{\left(z-\Omega_{0}\right)^{2}+(\Delta \Omega)^{2}}=\int_{-R}^{+R} \frac{e^{i(z t+\phi)} d z}{\left(z-\Omega_{0}\right)^{2}+(\Delta \Omega)^{2}}+\int_{C_{R}} \frac{e^{i(z t+\phi)} d z}{\left(z-\Omega_{0}\right)^{2}+(\Delta \Omega)^{2}}
$$

calculada sobre o contorno mostrado na figura C.1.

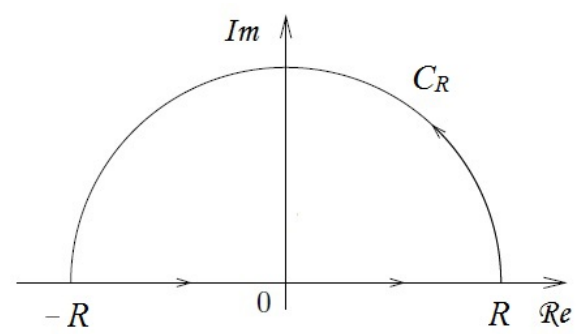

Figura C.1: Contorno empregado no cálculo da integral de uma variável complexa. [46] 
A primeira integral de $J$ pode ser reescrita de forma conveniente através de uma substituição da forma $z^{\prime}=z-\Omega_{0}$, como segue

$$
J=\oint_{C} \frac{e^{i(z t+\phi)} d z}{\left(z-\Omega_{0}\right)^{2}+(\Delta \Omega)^{2}}=\oint_{C} \frac{e^{i\left[\left(z^{\prime}+\Omega_{0}\right) t+\phi\right]} d z^{\prime}}{z^{\prime 2}+(\Delta \Omega)^{2}}=e^{i\left(\Omega_{0} t+\phi\right)} \oint_{C} \frac{e^{i z^{\prime} t} d z^{\prime}}{\left(z^{\prime}+i \Delta \Omega\right)\left(z^{\prime}-i \Delta \Omega\right)}
$$

Tal integração apresenta uma única singularidade $\left(z^{\prime}=i . \Delta \Omega\right)$ dentro da área delimitada pelo semicírculo $C$, logo, a mesma pode ser calculada pelo Teorema do Resíduo[47]

$$
\begin{gathered}
J=2 \pi i \operatorname{Res} f(i . \Delta \Omega)=2 \pi i e^{i\left(\Omega_{0} t+\phi\right)} \frac{e^{-\Delta \Omega t}}{2 i \Delta \Omega} \\
J=\frac{\pi}{\Delta \Omega} e^{-\Delta \Omega t} e^{i\left(\Omega_{0} t+\phi\right)}
\end{gathered}
$$

Notemos que a função $e^{i z}$ é limitada no semiplano superior, pois $e^{i z}=e^{i(x+i y)}=$ $e^{-y} e^{i x}$ e $\left|e^{i x}\right|=1$ (para todo $\mathrm{x}$ real), enquanto que $\left|e^{-y}\right| \leqslant 1$ (para todos os y nãonegativos). Daí obtemos a seguinte estimativa para a integral em $C_{R}$ :

$$
\left|\int_{C_{R}} \frac{e^{i(z t+\phi)} d z}{\left(z-\Omega_{0}\right)^{2}+(\Delta \Omega)^{2}}\right| \leqslant(\pi R) \frac{1}{R^{2}} \approx \frac{1}{R}
$$

Portanto, no limite de $R$ tendendo a infinito, segue que

$$
\lim _{R \rightarrow \infty} \int_{C_{R}} \frac{e^{i(z t+\phi)} d z}{\left(z-\Omega_{0}\right)^{2}+(\Delta \Omega)^{2}}=0
$$

Consequentemente, quando $R \rightarrow \infty$ em (C.5), temos que

$$
\int_{-\infty}^{+\infty} \frac{e^{i(z t+\phi)} d z}{\left(z-\Omega_{0}\right)^{2}+(\Delta \Omega)^{2}}=\frac{\pi}{\Delta \Omega} e^{-\Delta \Omega t} e^{i\left(\Omega_{0} t+\phi\right)}
$$

Como a integração anterior ocorre sobre o eixo real $(z \in \mathbb{R})$, segue da expansão em seno e cosseno da exponencial complexa, que a parte real de (C.10) é dada por

$$
\int_{-\infty}^{+\infty} \frac{\cos (z t+\phi) d z}{\left(z-\Omega_{0}\right)^{2}+(\Delta \Omega)^{2}}=\frac{\pi}{\Delta \Omega} e^{-\Delta \Omega t} \cos \left(\Omega_{0} t+\phi\right)
$$

Como $z$ é uma variável muda, podemos trocá-la por $\Omega$ e substituir tal resultado em (C.4), resultando em:

$$
I=\int_{-\infty}^{+\infty} \cos (\Omega t+\phi) d P(\Omega)=e^{-\Delta \Omega t} \cos \left(\Omega_{0} t+\phi\right)=e^{-t / T_{2}^{*}} \cos \left(\Omega_{0} t+\phi\right)
$$


onde $T_{2}^{*}=\frac{\hbar}{\mu_{B} B \Delta g_{e}}$ é o tempo de defasagem de spin do conjunto de ilhas.

Portanto, para obtermos a resposta do conjunto de ilhas quânticas no sinal de TRFR basta efetuarmos a substituição de $\cos (\Omega t+\phi)$ por $e^{-t / T_{2}^{*}} \cos \left(\Omega_{0} t+\phi\right)$ e $\Omega$ por $\Omega_{0}$ nos resultados do capítulo 4 . . 


\section{Apêndice D}

\section{Artigo Publicado e Apresentações em Conferências}

Nas páginas seguintes são apresentados, conforme a legenda abaixo, os trabalhos realizados durante o período de vigência do Mestrado.

Página 66-69 - Artigo publicado em Physical Review B; Título: Model for the light-induced magnetization in singly charged quantum dots; Publicado em 23 de Fevereiro de 2015; São Paulo - Brasil.

Página 70 - Apresentação de Pôster em International Conference on Nonlinear Optics and Excitation Kinetics in Semiconductors (NOEKS); 22-25 de Setembro de 2014, Bremen - Alemanha.

Página 71 - Apresentação Oral em XXXVII Encontro Nacional de Física da Matéria Condensada; 12-16 de Maio de 2014, Costa do Sauípe-BA, Brasil.

Página 72 - Apresentação Oral em IV Encontro Mario Schenberg, Departamento de Física dos Materiais e Mecânica; Instituto de Física - Universidade de São Paulo; São Paulo - Brasil.

Página 73 - Apresentação Oral em 17th Brazilian Workshop on Semiconductor Physics; 3-8 de Maio de 2015, Uberlândia-MG, Brasil. 


\title{
Model for the light-induced magnetization in singly charged quantum dots
}

\author{
A. B. Henriques and R. C. Cordeiro \\ Instituto de Física, Universidade de São Paulo, Caixa Postal 66318, 05314-970 São Paulo, Brazil \\ P. M. Koenraad and F. W. M. Otten \\ Department of Applied Physics, Eindhoven University of Technology, P.O. Box 513, NL-5600 MB Eindhoven, The Netherlands

$$
\text { M. Bayer }
$$ \\ Experimentelle Physik II, Technische Universität Dortmund, D-44221 Dortmund, Germany \\ and Ioffe Physical-Technical Institute, Russian Academy of Sciences, 194021 St. Petersburg, Russia \\ (Received 6 December 2014; revised manuscript received 4 February 2015; published 23 February 2015)
}

\begin{abstract}
Magnetization is induced in an ensemble of quantum dots, each charged with a single electron, when it is illuminated with a short circularly polarized light pulse that is resonant with the fundamental energy gap of the quantum dots. In this investigation, a quantum-mechanical model for the light-induced magnetization is presented. The phase of the magnetization precession as a function of the strength of the magnetic field in a Voigt geometry is in excellent agreement with experimental data measured on (In, Ga)As singly charged quantum dot ensembles. It is demonstrated that the precession of the hole in the trion plays a vital role because it determines the amplitude and phase of the magnetization precession. The model could also be easily extended to describe positively charged quantum dots. We also suggest that our theory, combined with measurements of the phase as a function of magnetic field, can be used as a technique to measure the resonant trion lifetime as a function of QD emission energy.
\end{abstract}

DOI: 10.1103/PhysRevB.91.081303

PACS number(s): 78.67.Hc, 78.20.Ek, 78.20.Jq, 78.20.Ls

Due to the long lifetime of the confined electron spin state, quantum dots (QDs) charged with a single electron are promising candidates for spin-based functional devices (see, for example, Ref. [1]). Of all methods, the manipulation and readout of the confined electron spin state using light is the fastest possible, and therefore has attracted the wide attention of researchers. Understanding the physics behind light control of magnetism is essential to advance this field and device applications based on it. A standard experiment that can be performed is to illuminate an ensemble of charged QDs with a short light pulse resonant with the fundamental energy gap of the QDs and measure, using the pump-probe technique, the magnetization of the ensemble as a function of time [2,3]. In this case a circularly polarized $\pi$ pulse induces a magnetization that precesses around a magnetic field [3,4] (see Fig. 1 for an example). The long-term magnetization that remains after the recombination of photoexcited particles is associated with the electrons that are permanently resident in the QD. The long-term oscillation of the $z$ component of the magnetization is described by $[2,5,6]$

$$
M_{z}(t)=-M_{z 0} e^{-t / T_{2}^{*}} \cos (\Omega t+\phi),
$$

where $M_{z 0}$ is the amplitude of the oscillation, $T_{2}^{*}$ the ensemble dephasing time [6], $\Omega=g_{e} \frac{e B}{2 m_{0}}$ the Larmor frequency, $g_{e}$ the electron $g$ factor, and $\phi$ the phase of the oscillation. The minus sign in (1) has been added for convenience of the phase definition. In this Rapid Communication we associate the magnitude of parameters $M_{0}$ and $\phi$ to the mechanism by which light creates magnetization in a QD ensemble. We present a quantum-mechanical theory for the magnetization process and compare its predictions with our pump-probe experimental data obtained on (In,Ga)As QDs. Our analysis shows that the coherence of the spin of the photoexcited holes plays a vital role in light-induced magnetization and spin coherence generation of QD ensembles.

When a magnetic field is applied along the $y$ direction, there will be a thermal distribution in the ensemble of both spin orientations parallel and antiparallel to the field. Taking the quantization axis along the photon wave vector (the $z$ direction), the spin wave function of the resident electron in a given QD will be given by

$$
\Psi\left(t=0_{-}\right)=\frac{1}{\sqrt{2}}|\uparrow \pm i \downarrow\rangle,
$$

where the plus (minus) sign corresponds to a spin parallel (antiparallel) to $B$. However, because the $z$ component of the precessing spin is independent of whether the initial state is parallel, or antiparallel, to $B$, for simplicity we shall henceforward consider only the plus sign in (2).

In the case when the lowest energy QD hole has a pure heavy hole character, optical selection rules in III-V semiconductors dictate that right- (left-) hand circularly polarized photons generate an electron-hole pair in which the electron spin is down (up). On the other hand, for resonant excitation, the photoexcited state must comply with the Pauli exclusion principle. For fundamental gap excitation, both the resident and the photoexcited electron will be in the same orbital state, therefore their spin states must differ, hence the photoexcited electron must have its spin antiparallel to the spin of the resident electron. Thus, immediately after the absorption of a right-hand circularly polarized photon, the wave function becomes

$$
\Psi\left(t=0_{+}\right)=\frac{1}{\sqrt{2}}|\Uparrow \downarrow \uparrow+i \downarrow\rangle,
$$




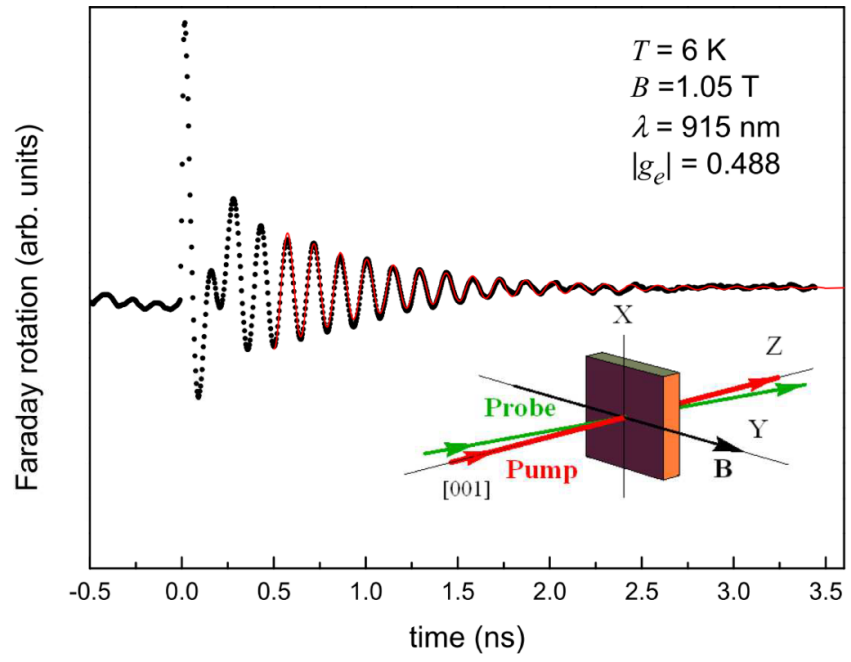

FIG. 1. (Color online) Pump-probe measurement of the magnetization oscillation. The Faraday rotation angle at any given instant is proportional to the magnetization of the QD ensemble along the optical axis. The continuous line was obtained by fitting the data for $t>0.5 \mathrm{~ns}$, using Eq. (1). The oscillation detected at negative delays is due to the mode-locking effect of spin coherence [4].

where the thick arrow represents the hole spin. The first (second) term in (3) will be addressed as the excited (unexcited) part of the spin wave function.

It was soon recognized that the electron spin returned from the trion at the instant of its recombination, $t=t_{r}$, should contribute to the long-term spin orientation of a quantum dot, however, for simplicity, mostly the strong dephasing limit has been hitherto analyzed theoretically, whereby the spin polarization is treated semiclassically $[2,4,7-10]$. There has been a report on a quantum-mechanical treatment of optical spin coherence generation in charged quantum dots, however, the model showed only limited agreement with the experimental data [11].

In the strong dephasing limit analyzed so far, the Larmor precession period $2 \pi / \Omega$ is much shorter than the average trion lifetime $\tau$, hence for typical (In,Ga)As QDs $\left(g_{e} \sim 0.5\right.$ [6] and $\tau \sim 0.4 \mathrm{~ns}$ [12]) one requires $B \gg 0.14 \mathrm{~T}$. For such fast dephasing, at the instant of trion recombination $t=t_{r}$, the excited part of the spin wave function becomes randomized. This implies that only the unexcited part of the spin wave function can contribute to the long-term magnetization, and because it started from a down state at $t=0$, the phase of the magnetization oscillation in (1) is zero, $\phi=0$. Thus, in the fast dephasing approximation, $\phi$ is always zero, irrespective of the strength of the applied magnetic field, electron or hole $g$ factor, trion lifetime, the specific QD singlet photoexcitation, and the QD band gap. This contradicts a previous investigation that has shown that the phase depends on the QD band gap [13].

Figure 2 shows the phase extracted by fitting the longterm $(t>0.5 \mathrm{~ns})$ magnetization oscillation, using (1), for a sample containing ten layers of negatively charged (In,Ga)As QDs, whose fundamental energy gap is resonant with a laser wavelength of $915 \mathrm{~nm}$. The average $g$ factor of the resident electron in the probed dots is $\left|g_{e}\right|=0.488$. The magnetization oscillation was probed both by time-resolved Faraday rotation

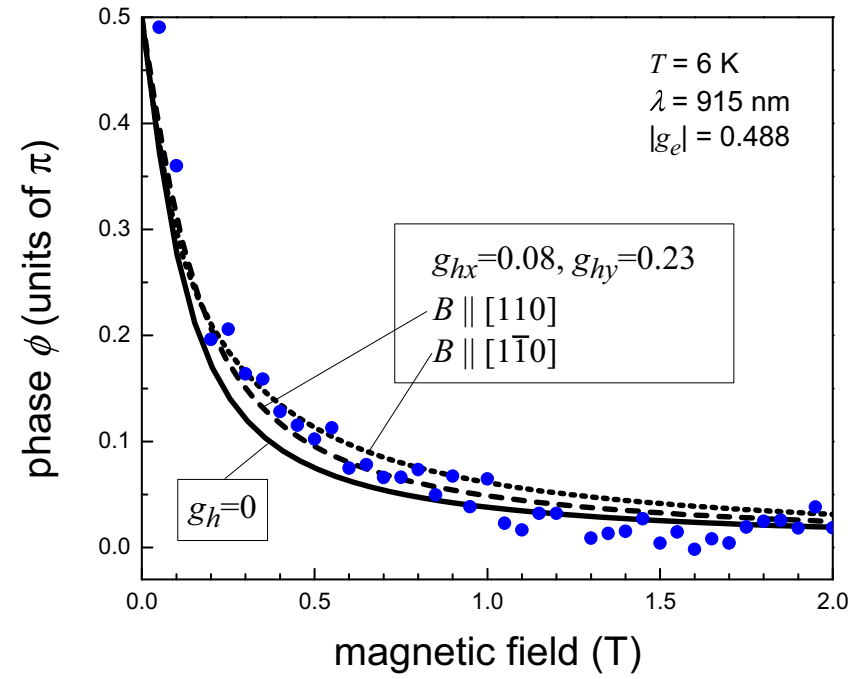

FIG. 2. (Color online) Phase $\phi$ of the long-term magnetization oscillations as a function of $B$, for a sample with $\left|g_{e}\right|=0.488$. Dots represent the experimental data. Lines represent theory for $\tau=0.2 \mathrm{~ns}$. Theory for $g_{h}=0$ (static hole) is shown by the solid line. Theory for $g_{h x}=0.08$ and $g_{h y}=0.23$ (taken from Ref. [15]) is shown by the dashed and dotted lines (corresponding to a magnetic field applied along [110] or [1ํㅣㄹ, respectively).

and ellipticity [8], yielding the same phase values. A second sample with $\left|g_{e}\right|=0.55$ was also measured, and showed a similar phase dependence on $B$.

It is obvious from Fig. 2 that the phase $\phi$ is not constant, as would be expected from the simplified picture, but changes from zero in the high field limit to $\phi=\pi / 2$ when the magnetic field intensity $B$ approaches zero, which is precisely when the fast dephasing approximation fails. Notice also that recently it was demonstrated that the hole spin coherence lifetime is typically 800 ns [14], which exceeds by three orders of magnitude the characteristic trion recombination time of $\tau$, which is in the nanosecond range [12]. Therefore, an accurate description of the phase of the long-term magnetization oscillation requires modeling the precession of both electrons and holes, which can be obtained by resolving the time-dependent Schrödinger equation. However, we will show that a simplified model, in which only the electrons precess while the hole does not (the static hole model, or the zero hole $g$-factor model), captures all the essential physics, and provides an excellent approximation. The static hole model has the enormous advantage of leading to concise expressions. In contrast, the expressions we derived using the complete model are cumbersome, because they depend on the precession of the hole and its anisotropic $g$ factor [15-17].

Let us then discuss the static hole model. The spin state of the resident electron at a given instant is determined by the time evolution operator $e^{-\frac{i}{\hbar} \mathcal{H} t}$, where $\mathcal{H}=\frac{1}{2} \hbar \Omega \sigma_{y}$ is the Zeeman energy for a magnetic field applied along the $y$ axis, and $\sigma_{y}$ is a Pauli matrix. Applying the time evolution operator to the initial spin state of (3), the spin function of the QD before trion recombination is found to be

$$
\Psi\left(t<t_{r}\right)=\frac{1}{\sqrt{2}}|\Uparrow \downarrow \uparrow+a \uparrow+b \downarrow\rangle,
$$


where $a=1-i \sin \frac{\Omega}{2} t$ and $b=i \cos \frac{\Omega}{2} t$. Taking into account that at $t=t_{r}$ the static hole captures a spin-down electron, for $t>t_{r}$ the time evolution operator gives

$$
\Psi\left(t>t_{r}\right)=\frac{1}{\sqrt{2}}|c \uparrow+d \downarrow\rangle,
$$

where $c=\cos \frac{\Omega}{2}\left(t-t_{r}\right)-i \sin \frac{\Omega}{2} t$ and $d=\sin \frac{\Omega}{2}\left(t-t_{r}\right)+$ $i \cos \frac{\Omega}{2} t$.

The $z$ component of the electronic magnetic moment in the QD is proportional to the expectation value of the Pauli matrix $\sigma_{z}$,

$$
\left\langle\sigma_{z}\left(t, t_{r}\right)\right\rangle=\left\langle\Psi\left|\sigma_{z}\right| \Psi\right\rangle,
$$

which can be evaluated for any $t$ and $t_{r}$, using spin wave functions (4) or (5). The average of (6) over $t_{r}$ is obtained using the decay law, whereby the probability that trion recombination occurs in the interval $\left[t_{r}, t_{r}+d t_{r}\right]$ equals $d P\left(t_{r}\right)=$ $e^{-t_{r} / \tau} d t_{r} / \tau$, where $\tau$ is the trion recombination time:

$$
\overline{\left\langle\sigma_{z}(t)\right\rangle}=\int_{0}^{\infty}\left\langle\sigma_{z}\left(t, t_{r}\right)\right\rangle e^{-t_{r} / \tau} \frac{d t_{r}}{\tau} .
$$

Finally, account must be taken of the $g$-factor inhomogeneity of the electrons in the QDs that contribute to the magnetization oscillation. The interpretation of the magnetization oscillation of a QD ensemble is almost insensitive to the exact mathematical formulation of the $g$-factor broadening function, as it has been demonstrated that either a Gaussian and a Lorentzian can be used to describe the data equally well, and yield essentially the same fitting values for the amplitude, frequency, phase, and dephasing [6]. Assuming a Lorentzian distribution of electron $g$ factors, with half width $\Delta g_{e} \ll g_{e}$, then for the inhomogeneous ensemble the result is

$$
\begin{aligned}
\overline{\left\langle\sigma_{z}(t)\right\rangle}= & -\frac{1}{2} \frac{e^{-t / \tau}}{1+\Omega^{2} \tau^{2}} \\
& -\frac{1}{2} \frac{\Omega \tau}{\sqrt{1+\Omega^{2} \tau^{2}}} e^{-t / T_{2}^{*}} \cos (\Omega t+\phi),
\end{aligned}
$$

where $T_{2}^{*}=1 /\left(\Omega g_{e} \Delta g_{e}\right)$ and

$$
\phi=\frac{\pi}{2}-\arctan \Omega \tau \text {. }
$$

The first term in (8) describes the $B \rightarrow 0$ limit in which a negative nonprecessing electronic magnetic moment is generated when a trion is created. It is associated with the unexcited part of the spin wave function, and vanishes when the trion recombines. The second term in (8) describes the precessing electronic magnetic moment associated with the superposition of the photoexcited and unexcited parts of the spin wave function. It is coincident with (1) and sets the amplitude $M_{z 0} \sim \frac{1}{2} \frac{\Omega \tau}{\sqrt{1+\Omega^{2} \tau^{2}}}$.

Equation (9) nicely reproduces the observed behavior, i.e., $\phi=0$ when $B$ is large and $\phi \rightarrow \frac{\pi}{2}$ when $B \rightarrow 0$. For intermediate values of $B$, (9) also reproduces the experimental results very well, as Fig. 2 shows.

The phase for the two limiting $B$ values can be understood from the spin vector diagram shown in Fig. 3. At small fields, when $\Omega \tau \ll 1$, the precession is very slow, hence at the instant of recombination, the unexcited precessing spin wave function gives rise to a vector, $\boldsymbol{S}_{u}$, that is rotated by a small angle with

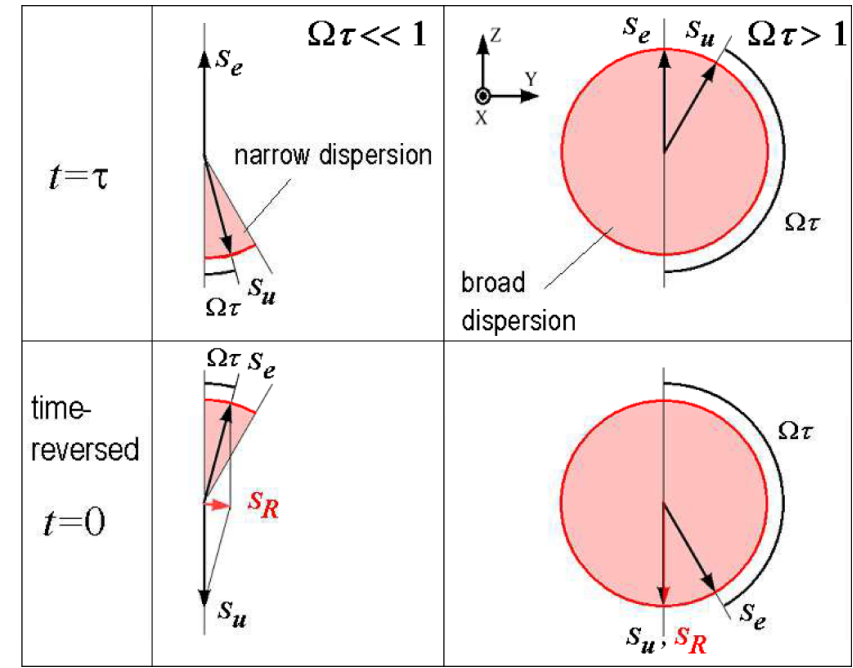

FIG. 3. (Color online) Schematic diagram of the expectation value of the spin vectors at the average instant of recombination, $t=\tau$ (top panels), and time reversed to $t=0$ (bottom panels). Panels on the left and on the right represent the vectors in the low and high magnetic field limits, respectively.

respect to its initial down direction. Moreover, the dispersion in the $S_{u}$ rotation angle among the dots, due to the random recombination times, will be small, because the precession is slow. The phase of the long-term spin oscillation is determined by the orientation of the average QD spin vector at $t=0$, which can be obtained by time reversing $\boldsymbol{S}_{u}$ and $\boldsymbol{S}_{e}$, and calculating their resultant at $t=0$. The time-reversed $S_{u}$ points down for all dots, whereas $\boldsymbol{S}_{e}$ will be rotated from the up direction by a small angle, and distributed over a small range of angles. As shown in Fig. 3, the resultant spin $\boldsymbol{S}_{R}$ is nearly perpendicular to $z$, meaning a phase of $\phi=\pi / 2$, and its modulus is small.

The same procedure can be applied to analyze the phase of the long-term magnetization oscillation for large $B$, when $\Omega \tau>1$. In this case, the precession is fast, and at the instant of recombination $S_{u}$ will be rotated by a large angle with respect to the down direction, and the dispersion in its rotation angle will also be large. Performing a time reversal to $t=0$, the $\boldsymbol{S}_{u}$ will still point down for all dots, and the large dispersion of rotation angles will be transferred to $S_{e}$, which then averages out to zero. Consequently, at large fields the time-reversed resultant magnetization of the QD points down, which gives a phase $\phi=0$, and the oscillation amplitude attains the maximum possible value. Therefore, the static hole model yields a vanishing amplitude and a phase of $\phi=\pi / 2$ when $B \rightarrow 0$, and a steady amplitude and a phase of $\phi=0$ when $B$ is large, which fully agrees with our experimental observations.

If we now allow for the hole to precess, then the timereversed precession angle of the excited part will be on average changed by $\Omega_{h} \tau$, where $\Omega_{h}=g_{h} \mu_{B} B$ is the hole precession and $g_{h}$ the hole $g$ factor. Hence, in the limiting cases of magnetic field strength, an account of the hole precession does not modify the predicted phase and amplitude of the magnetization oscillation obtained using the static hole model. The hole precession, however, does have a small effect on the phase in the intermediate range of magnetic fields, as Fig. 2 shows. The small differences between the theoretical curves 
shown in Fig. 2 will probably be washed out if ensemble inhomogeneity in the hole $g$ factor is taken into account.

The fitting of the long-term oscillation phase versus magnetic field is done using a single parameter, $\tau$. In our deduction of $\tau$, all external parameters were fixed, except for the magnetic field strength, which was varied in the range $0-1.5 \mathrm{~T}$. For such small fields, the wave function of the confined electrons and hole in the photoexcited QDs are essentially unchanged from their $B=0$ values, because the Lorentz forces acting on the confined particles are much smaller than the Coulomb forces associated with the QD potential, as evidenced by a negligible diamagnetic shift of the exciton photoluminescence (PL). Differential transmission studies on similar quantum dots have shown that the decay time of the ground state population does not changes for the weak magnetic fields applied here. Therefore, $\tau$ can be safely taken as an accurate estimate of the trion lifetime.

The lifetime estimated in such a way corresponds to a trion that recombines at the same energy as the excitation light (i.e., a resonant trion). This contrasts to the typical trion lifetime obtained from conventional time-resolved photoluminescence, whereby resonant excitation is difficult due to scattered light, hence nonresonant excitation is used, in which case the lifetime is strongly influenced by relaxation and possibly dark exciton formation. Differential transmission measurements [12] have estimated the nonresonant trion lifetime to be twice longer than the fully resonant one, which would then bring the value of $\tau=0.2 \mathrm{~ns}$ obtained in this work to about $\tau \sim 0.4 \mathrm{~ns}$, which agrees with typical values for (In, Ga)As/GaAs QDs [18].

In conclusion, we have developed a quantum-mechanical model of light-induced magnetization in a charged QD ensemble that takes into account the spin coherence of the photoexcited holes. The model is based on an intuitive and transparent approach, i.e., a solution of the time-dependent Schrödinger equation for the spin wave functions and subsequently averaging over the QD ensemble, and leads to a concise analytical expression for the magnetization oscillation in the presence of a magnetic field. The phase of the magnetization oscillation gradually evolves from $\phi=\pi / 2$ at $B=0$ to $\phi=0$ when $B$ is large. The theory is in excellent agreement with experimental data obtained on a self-assembled singly negatively charged (In,Ga)As/GaAs QD ensemble. The theory given here gives insight into spin coherence generation, it is relevant not only to self-assembled but also to QD systems in general, including colloidal QDs, and it can be easily extended to $p$-doped dots using the approach and building blocks given in our model.

Furthermore, the method used by us offers a technique to measure the lifetime of a trion in a QD under resonant excitation. Our technique could be employed to obtain an accurate estimate of the resonant trion lifetime as a function of QD emission energy.

A.B.H. acknowledges financial support provided by $\mathrm{CNPq}$ (Projects No. 401694/2012-7 and No. 304685/2010-0), FAPESP (Project No. 2012/23406-0), and Laboratório de Microfabricação (LMF)/Laboratório Nacional de Nanotecnologia (LNNano). M.B. acknowledges the support by the Deutsche Forschungsgemeinschaft (ICRC-TRR160) and the Russian Ministry of Education and Science (Project No. 14.Z50.31.0021)
[1] Semiconductor Quantum Bits, edited by F. Henneberger and O. Benson (Pan Stanford, Singapore, 2008).

[2] S. G. Carter, S. C. Badescu, and A. S. Bracker, Phys. Rev. B 81, 045305 (2010).

[3] A. Greilich, R. Oulton, E. A. Zhukov, I. A. Yugova, D. R. Yakovlev, M. Bayer, A. Shabaev, A. L. Efros, I. A. Merkulov, V. Stavarache, D. Reuter, and A. Wieck, Phys. Rev. Lett. 96, 227401 (2006).

[4] A. Greilich, D. R. Yakovlev, A. Shabaev, Al. L. Efros, I. A. Yugova, R. Oulton, V. Stavarache, D. Reuter, A. Wieck, and M. Bayer, Science 313, 341 (2006).

[5] E. A. Zhukov, D. R. Yakovlev, M. Bayer, M. M. Glazov, E. L. Ivchenko, G. Karczewski, T. Wojtowicz, and J. Kossut, Phys. Rev. B 76, 205310 (2007).

[6] A. Schwan, B.-M. Meiners, A. B. Henriques, A. D. B. Maia, A. A. Quivy, S. Spatzek, S. Varwig, D. R. Yakovlev, and M. Bayer, Appl. Phys. Lett. 98, 233102 (2011).

[7] S. Varwig1, A. Greilich, D. R. Yakovlev, and M. Bayer, Phys. Status Solidi B 251, 1892 (2014).

[8] I. A. Yugova, M. M. Glazov, E. L. Ivchenko, and Al. L. Efros, Phys. Rev. B 80, 104436 (2009).

[9] M. M. Glazov, I. A. Yugova, S. Spatzek, A. Schwan, S. Varwig, D. R. Yakovlev, D. Reuter, A. D. Wieck, and M. Bayer, Phys. Rev. B 82, 155325 (2010).
[10] A. Shabaev, Al. L. Efros, D. Gammon, and I. A. Merkulov, Phys. Rev. B 68, 201305 (2003).

[11] M. V. Gurudev Dutt et al., Phys. Rev. Lett. 94, 227403 (2005).

[12] H. Kurtze, J. Seebeck, P. Gartner, D. R. Yakovlev, D. Reuter, A. D. Wieck, M. Bayer, and F. Jahnke, Phys. Rev. B 80, 235319 (2009).

[13] A. B. Henriques, A. Schwan, S. Varwig, A. D. B. Maia, A. A. Quivy, D. R. Yakovlev, and M. Bayer, Phys. Rev. B 86, 115333 (2012).

[14] F. Fras, B. Eble, B. Siarry, F. Bernardot, A. Miard, A. Lemaître, C. Testelin, and M. Chamarro, Phys. Rev. B 86, 161303(R) (2012).

[15] A. Schwan, B.-M. Meiners, A. Greilich, D. R. Yakovlev, M. Bayer, A. D. B. Maia, A. A. Quivy, and A. B. Henriques, Appl. Phys. Lett. 99, 221914 (2011).

[16] R. Roloff, T. Eissfeller, P. Vogl, and W. Pötz, New J. Phys. 12, 093012 (2010).

[17] C. Testelin, F. Bernardot, B. Eble, and M. Chamarro, Phys. Rev. B 79, 195440 (2009).

[18] T. Berstermann, T. Auer, H. Kurtze, M. Schwab, D. R. Yakovlev, M. Bayer, J. Wiersig, C. Gies, F. Jahnke, D. Reuter, and A. D. Wieck, Phys. Rev. B 76, 165318 (2007). 


\title{
Light-Induced Coherent Spin Precession in Charged Quantum Dots
}

\author{
A.B. Henriques', R.C. Cordeiro', F.W.M. van Otten'2, P.M. Koenraad² and M. Bayer ${ }^{3}$
}

'Instituto de Fisica, Universidade de Sijo Paulo, Sijo Paulo, Brazil

2Department of ApplledPhysics, Eindhoven University of Technology, Eindhoven, The Netherlands

"Experimentelle Physik II, Technische Universität Dortmund, Dortmund, Germany

\section{Why is spin coherence needed?}

- An ensemble of self-assembled QDs, each with a resident electron has zero magnetic moment, because spins in different $\mathrm{QD}$ s are uncorrelated;

- Optical excitation allows to generate a coherent spin-polarized ensemble with a robust measureable magnetic moment

- A measurable quantity allows opens access to probing the spin physics of quantum dots, as well as bo possible practical applications in spintronic devices.

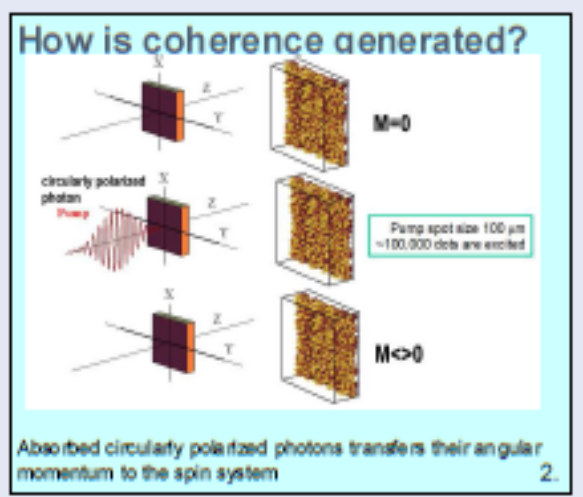

\section{Consequences of random electron} capture

- Only the unexcited spin function component contributes to the magnetic moment of the ensemble

- All excited dots contribute to the ensemble spin with a spin that pointed down (i.e., in the $Z$ directon) at $\mathrm{t}=0$;

- The phase of the precessing magnetzation is $\pi$, irrespective of magnetic field intensity, $Q D$ energy gap, etc

- This is in conflict with experiments! [2] 4 .

\section{Simple result for $g_{h}=0$}

$$
\begin{gathered}
\left\langle\sigma_{2}(t)\right\rangle=-\frac{1}{2} e^{-t / \tau} \frac{1}{1+\Omega^{2} \tau^{2}}-\frac{1}{2} \frac{\Omega \tau}{\sqrt{1+\Omega^{2} T^{2}}} \cos (\Omega t+\phi) \\
\phi=\frac{\pi}{2}-\arctan \Omega \tau
\end{gathered}
$$

- First term: transient. Hole captures same spin that was generated in the trion.

Consider $\mathrm{B}=0$ then second term is zero. The exponential decay is because magnefzation retums to zero once excited spin is captured.

- Second term: see next slide

\section{General predictions}

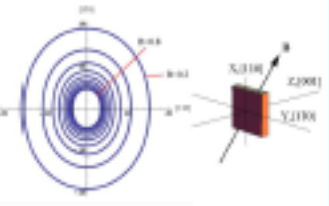

- Magnetic field anisotropy of the phase

- Appearance of beating trequencies

- Pumping into excited (triplet) states generates multiple harmonics

\section{But does the hole really capture a} random electron?

One would expect the hole to capture a random electron under one of these conditions:

(1) the hole spin is a poor quantum number

(2) the hole is a good quantum number, but precesses fast (meaning many precession periods within the trion lifetme).

But the hole spin is a good quantum number [1], and (2) is rarely sat sfed because $\tau$ is short. Reexamination is therefore needed.

5.

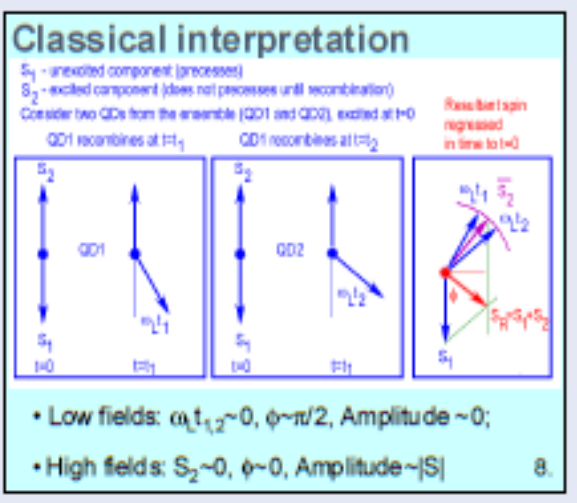

Conclusions
-At low magnetic fields the phase of
ensemble magnetzation precession differs
by $\pi 2$ from the high feld limit
- Amplitude of magnetization is strongly
dependent on $B$
- Theory was extended to calculate the phase,
amplitude and beatings for pumping
resonant with triplet states of $Q D$, which
still remains to be tested.

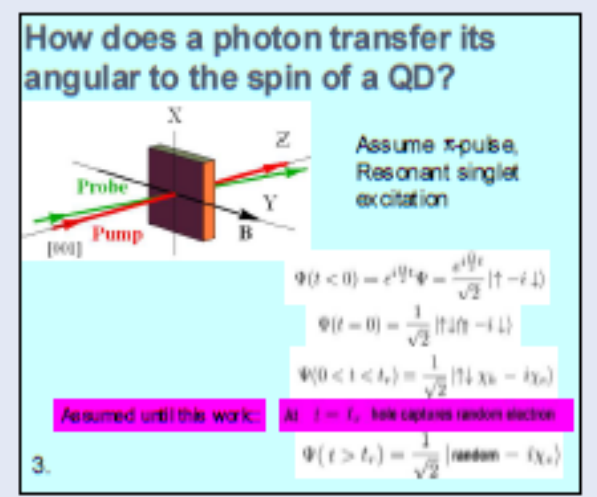

Full quantum mechanical treatment

$$
\begin{aligned}
& x=\operatorname{swn}_{3} \theta \frac{3}{\frac{3}{2}} \\
& x \in(t)=e^{-\frac{\mu \alpha}{\gamma}} x \in(0)
\end{aligned}
$$

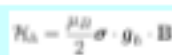

$$
\begin{aligned}
& \left.x_{n}(t)-e^{-\frac{1}{3} x_{n}} \mid t\right\} \\
& \langle\sigma(t)\rangle=\int x_{i}^{*} \sigma x_{e} d t \\
& d P\left(t_{r}\right)=\frac{e^{-t, r i r} \text { ift, }}{r} \\
& \overline{\left\langle\sigma_{s}(T)\right\rangle}=\int\left(\sigma_{t}(t)\right) d P\left(t_{\varphi}\right)
\end{aligned}
$$

6.

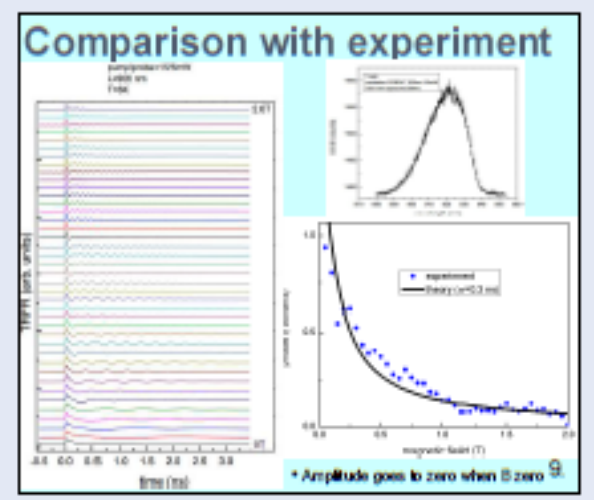

\section{References}

[1]. S. Varwig, A. Greilich, D. R. Yakovlev, M. Bayer, Phys. Stat. Solidi B 20, 1 (2014)

[2]. A. B. Henriques, A. Schwan, S. Varwig, A. D. B. Maia, A. A. Quivy, D. R. Yakovlev, and M. Bayer, Phys. Rev. B 86, 115333 (2012)

\section{Acknowledgements}

Financial support by Brazl lian Agencies FAPESP and CNPq is gratefully acknowledged 


\title{
Optical spin orientation and coherent Larmor precession of electrons and holes in charged quantum dot ensembles
}

\author{
A. B. Henriques, Renan Carlos Cordeiro \\ Instituto de Fisica da Universidade de Sao Paulo
}

An electron or hole spin in a semiconductor quantum dot (QD) is a strong candidate as a quantum bit for quantum information applications. Optical manipulation of spin states is of particular interest since it can be performed on ultrafast time scales.

It is well known that an ensemble of self-assembled negatively charged QDs can be coherently spin-polarized using picosecond pulses of circularly polarized photons, resonant with the fundamental energy gap of the QD. Given that prior to excitation the spin in a QD is in-plane oriented, the selection rules for QD dipole electronic transitions imply that the light pulse drives the QD into a state whose spin wave function is a superposition of an excited component (the trion) and an unexcited component (a single electron). At the instant of trion recombination, the excited spin function component is converted into a single electron spin function, which is superimposed on the unexcited one. Up to now, it has been assumed by researchers that recombination randomizes the excited spin component (which averages out to zero), because the recombination process is stochastic. Therefore all spin coherence observed must be associated to the unexcited spin function component, and the Larmor precession phase of QD spins is determined solely by the instant the light pulse hits the QD. In reality, though, the trion recombination is governed by the universal law of radioactive decay, i. e. a most likely instant of recombination. Hence the spin component created at trion recombination is not random, it does not average out to zero, and it affects the precession. In this work, this scenario is incorporated via a full quantum-mechanical model. Both singlet and triplet resonances, were investigated. The amplitude, frequency spectrum, and phase of the coherent precession of resident electrons in the QD ensemble was deduced, and it is found that in III-V the phase displays a $15 \%$ anisotropy when the Voigt magnetic field is rotated perpendicular to the [001] growth axis. The phase shows a steep dependence on the magnetic field. These predictions agree with our published experimental data, obtained at our Laboratory at USP (time resolved Faraday rotation in (In, Ga)As/GaAs negatively charged QDs, using picosecond pump-probe experiments). More experiments are needed to confirm the interesting picture that arises, including the hypothetical case when the precession of the hole and electron are described by similar Larmor frequencies. 


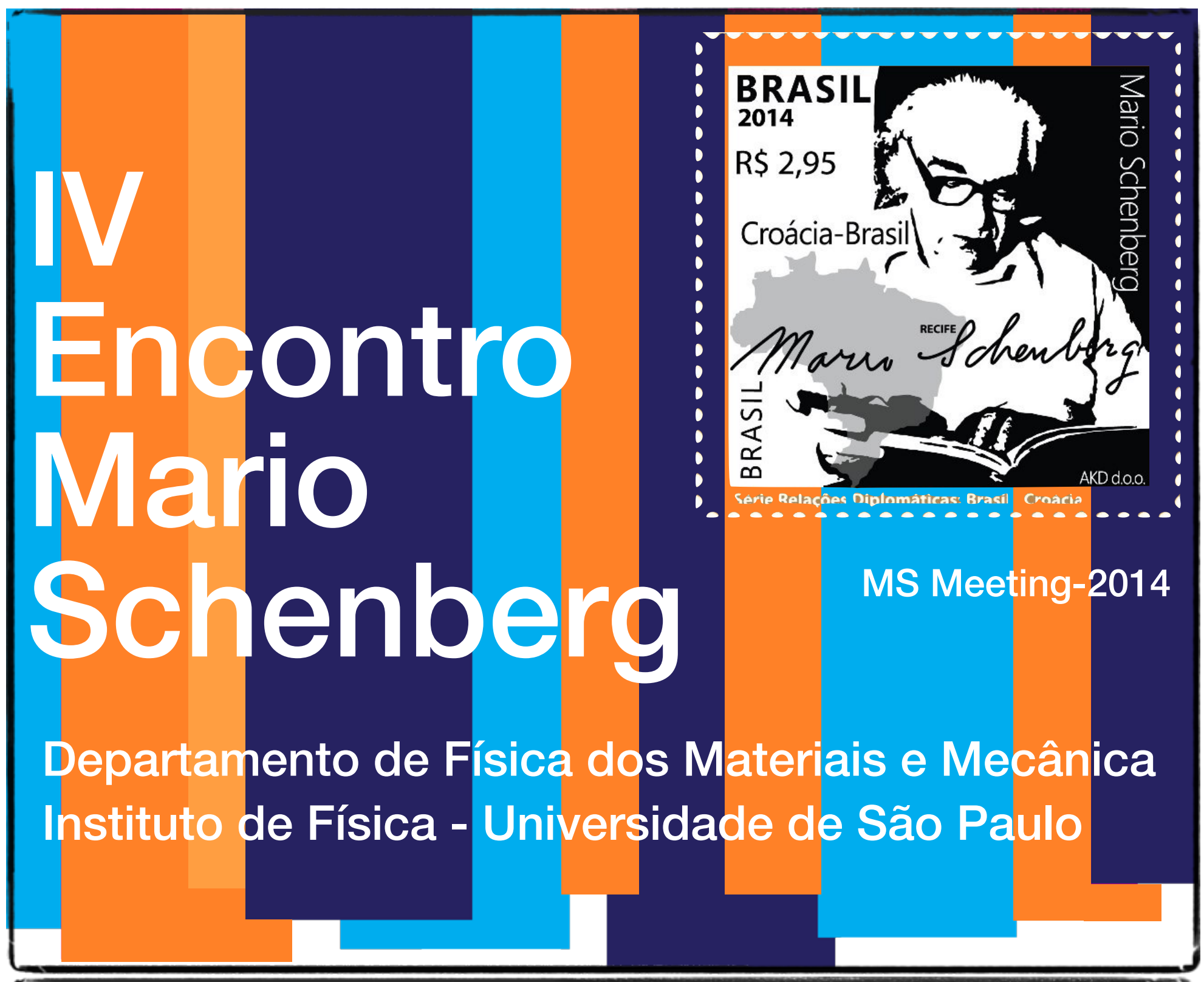

Local: O Departamento de Física dos Materiais e Mecânica (DFMT) convida todos a participarem do Encontro Mario Schenberg 2014. Este incluirá uma sessão em homenagem ao Prof.

Schenberg, figura importante no desenvolvimento da física dos materiais, que faria 100 anos em 2014. Um selo postal comemorativo será apresentado pelos Correios. A pesquisa atual na área de Física dos Materiais será mostrada em sessões orais por professores e alunos.

de Moraes

Data

Quarta-feira 3 de Dezembro 8:30-19:00 hs.

DFMT/IFUSP

8:30 Abertura e apresentação de selo em homenagem a Mario Schenberg.

9:00 Homenagem a Mario Schenberg. Palestrantes: Professores S. Salinas e E. Hamburger.

Apresentações Científicas

10:20 Prof. Adalberto Fazzio. Isolantes topológicos via cálculos de primeiros princípios.

10:50 Prof. Rafael Freitas. Frustração e transições de fase em altos campos magnéticos e baixas temperaturas.

11:20 Prof. Luis Gregório Dias. Efeito Kondo em grafeno desordenado.

11:50 Prof. Felix Hernandez. Spintrônica em semicondutores no LNMS.

portal.if.usp.br/fmt

14:00 Prof. Marília Caldas. Electronic properties of organic semiconductor polymers: how does conjugation length impacts ionization potentials and gaps?

14:30 Apresentações orais de alunos e pós-doutorandos do DFMT

17:50 Mesa Redonda: "O papel do físico". Professores Adalberto Fazzio (chair), António José Roque da Silva (LNLS), Marcia Barbosa (UFRGS), Vera Bohomoletz Henriques (IFUSP), Paulo Nussenzveig (IFUSP).

18:50 Encerramento. Prof. A. Fazzio, Diretor do IFUSP. 


\title{
Triplet state spin coherence generation in singly-charged quantum dot ensembles by picosecond light pulses
}

\author{
A. B. Henriques and R. C. Cordeiro \\ Instituto de Física, Universidade de São Paulo, C.P. 66318, 05315-970 São Paulo, Brazil
}

In ensembles of quantum dots (QDs) containing a single resident charge (a hole, or an electron), the magnetic moments of the dots (due to the resident charge carriers) are uncorrelated, thus the overall magnetization averages out to zero. However, a circularly polarized light pulse resonant with the fundamental quantum dot energy gap can induce a magnetization [1]. This is a consequence of angular momentum conservation: the angular momentum of the absorbed photon is transferred to the charge carriers, and it ultimately ends up orienting the spin of the resident carriers in the direction of propagation of the incident light. Such a photoinduced magnetization will Larmor-precess around a magnetic field, if one is present. Recently [2], we have shown that the time-dependent magnetic state of a single dot and of the ensemble can be obtained exactly, by resolving the time-dependent Schrödinger equation for the light-carrier interaction. The theory produces the amplitude and phase of the magnetization precession, which is found to be in excellent agreement with experimental observations. In Ref. [2], the excitation light was assumed resonant with the fundamental energy gap of the QDs, whereby only singlet excitations are generated.

In this work, we consider triplet excitations, when the excitation light is resonant with overtone QD states. In III-V negatively charged QDs, when the coherent precession of the hole must be taken into account, we investigated the limit of static (non-precessing) hole, which corresponds to a hole with a zero gyromagnetic factor. The static hole result is compared to the exact solution (i. e. incorporating a non-zero anisotropic hole g-factor). We demonstrate that a measurement of the phase of the long-term magnetization induced by triplet state pumping, as a function of applied magnetic field in the Voigt geometry, can be used to provide an accurate estimate of the excited state lifetime dependence on the QD energy bandgap. The theory given here gives the deepest insight into spin coherence generation that has been accomplished so far, it is relevant not only to self-assembled but also to single QDs or QD systems in general, including colloidal QDs, and it can be easily extended to p-doped dots using the approach and building blocks given in our model.

A.B.H. acknowledges financial support provided by CNPq (Projects 401694/2012-7 and 304685/2010-0), FAPESP (Project 2012/23406-0), and Laboratório de Microfabricação (LMF)/Laboratório Nacional de Nanotecnologia (LNNano).

[1] A. Greilich, D. R. Yakovlev, A. Shabaev, A., Al. L. Efros, I. A. Yugova, R. Oulton, V. Stavarache, D. Reuter, A.Wieck, and M. Bayer, Science 313, 341-345 (2006).

[2] A. B. Henriques, R. C. Cordeiro, P. M. Koenraad, F. W. M. Otten, M. Bayer, Phys. Rev. (Rapid Communications), 2015 (at press). 


\section{Referências Bibliográficas}

[1] I.S. Oliveira and V.L.B. de Jesus. Introdução à Fúsica do Estado Sólido. Livraria da Física, 2011.

[2] M.A. Nielsen and I.L. Chuang. Computação Quântica e Informação Quântica. Bookman, 2005.

[3] S. Fafard, K. Hinzer, S. Raymond, M. Dion, J. McCaffrey, Y. Feng, and S. Charbonneau. Science, 274:1350, 1996.

[4] F. Heinrichsdorff, M.H. Mao, N. Kirstaedter, A. Krost, D. Bimberg, A.O. Kosogov, and P. Werner. Appl. Phys. Lett., 71:22, 1997.

[5] Z. Yuan, B.E. Kardynal, R.M. Stevenson, A.J. Shields, C.J. Lobo, K. Cooper, N.S. Beattie, D.A. Ritchie, and M. Pepper. Sciences (N.Y.), 295:102, 2002.

[6] K. Yano, T. Ishii, T. Hashimoto, T. Kobayashi, F. Murai, and K. Seki. IEEE Trans. Electron Devices, 41:1628, 1994.

[7] D. Loss and D.P. DiVincenzo. Quantum computation with quantum dots. Phys.Rev. A, 57, 1998.

[8] S. Chakrabarti, M.A. Holub, P. Bhattacharya, T.D. Mishima, M.B. Santos, M.B. Johnson, and D.A. Blom. Spin-polarized light-emitting diodes with Mn-doped InAs quantum dot nanomagnets as a spin aligner. Nano Lett, 5, 2005. 
[9] G. Prinz. Spin-polarized transport. Physics Today, 48, 1995.

[10] A. Tartakovskii, editor. Quantum Dots: Optics, Electron Transport and Future Applications. Cambrigde University Press, 2012.

[11] D. J. Griffiths. Mecânica Quântica. Pearson, 2011.

[12] M.I. Dyakonov, editor. Spin Physics in Semiconductors. Springer-Verlag, 2008.

[13] F. Meier and B. Zakharchenya. Optical Orientation: Modern Problems in Condensed Matter Sciences. North-Holland, 1984.

[14] D. J. Griffiths. Eletrodinâmica. Pearson, 3 edition, 2011.

[15] J. Frenkel. Princípios de Eletrodinâmica Clássica. Editora da Universidade de São Paulo (Edusp), 1996.

[16] C.P. Slichter. Principles of Magnetic Resonance, volume 1 of Springer Series in Solid-State Sciences. Springer-Verlag, 3 edition, 1996.

[17] D.J. Griffiths. Hyperfine splitting in the ground state of hydrogen. American Journal Physics, 50(8):698-703, 1982.

[18] S Blundell. Magnetism in condensed matter. Oxford Master Series in Condensed Matter Physics. Oxford University Press, 2001.

[19] D. J. Klauser. Hyperfine interaction and spin decoherence in quantum dots. PhD thesis, University of Basel, Faculty of Science, 2008.

[20] J. Schleimann, A. Khaetskii, and D. Loss. Electron spin dynamics in quantum dots and related nanostructures due to hyperfine interaction with nuclei. Journal of Physics: Condensed Matter, 15:R1809-R1833, 2003. 
[21] I.A. Merkulov, Al.L. Efros, and M. Rosen. Electron spin relaxation by nuclei in semiconductor quantum dots. Physical Review B, 65, 2002.

[22] Guido Burkard, Daniel Loss, and David P. DiVincenzo. Coupled quantum dots as quantum gates. Phys. Rev. B, 59, 1999.

[23] J. Beyer. Spin properties in InAs/GaAs based quantum structures. PhD thesis, Linköpings universitet, Schweden, 2012.

[24] S.A. Wolf, D.D. Awschalom, R.A. Buhrman, J.M. Daughton, S. von Molnár, and M.L. Roukes. Spintronics: A spin-based electronics vision for the future. Science, 294, 2001.

[25] D.D. Awschalom and J.M. Kikkawa. Electron spin and optical coherence in semiconductors. Physics Today, 1999.

[26] J.M. Kikkawa, I.P. Smorchkova, N. Samarth, and D.D. Awschalom. Roomtemperature spin memory in two-dimensional electron gases. Science, 227, 1997.

[27] A.P. Heberle, J.J. Baumberg, and K. Kohler. Ultrafast coherent control and destruction of excitons in quantum wells. Phys. Rev. Lett., 75, 1995.

[28] G. Slavcheva and P. Roussignol, editors. Optical Generation and Control of Quantum Coherence in Semiconductor Nanostructures. Nanoscience and Technology. Springer-Verlag, 2010.

[29] S.G. Carter, S.C. Badescu, and A.S. Bracker. Electron spin polarization and detection in InAs quantum dots through p-shell trions. Phys. Rev. B, 81, 2010.

[30] A. Schwan, B.M. Meiners, A. Greilich, D. R. Yakovlev, A.D.B. Bayer, M. Maia, A. Quivy, and A.B. Henriques. Anisotropy of electron and hole g-factors in (In, Ga)As quantum dots. Applied Physics Letters, 99, 2011. 
[31] E. A. Zhukov, D. R. Yakovlev, M. Bayer, M. M. Glazov, E. L. Ivchenko, G. Karczewski, T. Wojtowicz, and J. Kossut. Spin coherence of a two-dimensional electron gas induced by resonant excitation of trions and excitons in $\mathrm{CdTe} /(\mathrm{Cd}, \mathrm{Mg}) \mathrm{Te}$ quantum wells. Phys. Rev. B, 76, 2007.

[32] A. Greilich, R. Oulton, E. A. Zhukov, I. A. Yugova, D. R. Yakovlev, M. Bayer, A. Shabaev, Al. L. Efros, I. A. Merkulov, V. Stavarache, D. Reuter, and A. Wieck. Optical control of spin coherence in singly charged (In, Ga)As/GaAs quantum dots. Phys. Rev. Lett., 96, 2006.

[33] G.D. Galgano. Orientação óptica de spin em semicondutores magnéticos - calcógenos de európio. PhD thesis, Universidade de São Paulo, 2012.

[34] P.L.J. Helgers. Optical generation of spin coherence in (In, Ga)As quantum dots. Master's thesis, Eindhoven University of Technology, 2013.

[35] A.B. Henriques, A. Schwan, S. Varwig, A.D.B. Maia, A.A. Quivy, D.R. Yakovlev, and M. Bayer. Spin coherence generation in negatively charged self-assembled (In, Ga)As quantum dots by pumping excited trion states. Physical Review B, $86,2012$.

[36] S. Varwig, A. Greilich, R. Yakovlev, and M. Bayer. Spin mode locking in quantum dots revisited. Physica Status Solidi B, 251(9):1892-1911, 2014.

[37] A. Greilich, R. Oulton, E.A. Zhukov, I.A. Yugova, D.R. Yakovlev, M. Bayer, A. Shabaev, Al. L. Efros, V. Stavarache, D. Reuter, and A. Wieck. Optical generation of spin coherence in single-charged (In, Ga)As/GaAs self-assembled quantum dots. Physica E, 35, 2006. 
[38] I.A. Yugova, A. Greilich, A. Zhukov, D.R. Yakovlev, M. Bayer, D. Reuter, and A.D. Wieck. Exciton fine structure in InGaAs/GaAs quantum dots revisited by pump-probe Faraday rotation. Phys. Rev. B, 75, 2007.

[39] T. Andlauer and P. Vogl. Electrically controllable g tensors in quantum dot molecules. Physical Review B, 79, 2009.

[40] A. Greilich, D.R. Yakovlev, and M Bayer. Optical Generation and Control of Quantum Coherence in Semiconductor Nanostructures, chapter 6 - Ensemble Spin Coherence, page 90. Nanoscience and Technology. Springer-Verlag, 2010.

[41] R. Roloff, T. Eissfeller, P. Vogl, and W. Pötz. Electric g tensor control and spin echo of a hole-spin qubit in a quantum dot molecule. New Journal of Physics, 2010.

[42] C. Testelin, F. Bernardot, B. Eble, and M. Chamarro. Hole spin dephasing time associated with hyperfine interaction in quantum dots. Phys. Rev. B, 79, 2009.

[43] E. Poem, Y. Kodriano, C. Tradonsky, N.H. Lindner, B.D. Gerardot, P.M. Petroff, and D. Gershoni. Accessing the dark exciton with light. Nature Physics, 6, 2010.

[44] H. Kurtze, J. Seebeck, P. Gartner, D.R. Yakovlev, D. Reuter, A.D. Wieck, M. Bayer, and F. Jahnke. Carrier relaxation dynamics in self-assembled semiconductor quantum dots. Phys. Rev. B, 80, 2009.

[45] T. Berstermann, T. Auer, H. Kurtze, M. Schwab, D. R. Yakovlev, M. Bayer, J. Wiersig, C. Gies, F. Jahnke, D. Reuter, and A. D. Wieck. Systematic study of carrier correlations in the electron-hole recombination dynamics of quantum dots. Phys. Rev. B, 76, 2007.

[46] E. Butkov. Mathematical Physics. Addison-Wesley Publishing Company, 1968. 
[47] G. Arfken, H.J. Weber, and F.E. Harris. Mathematical Methods For Physicists. Elsevier, 2013. 